\title{
Multiplicity of Galactic Cepheids and RR Lyrae stars from Gaia DR2
}

\section{Resolved common proper motion pairs ${ }^{\star}$}

\author{
Pierre Kervella ${ }^{1}$, Alexandre Gallenne ${ }^{2}$, Nancy Remage Evans ${ }^{3}$, Laszlo Szabados ${ }^{4}$, Frédéric Arenou ${ }^{5}$, \\ Antoine Mérand ${ }^{6}$, Nicolas Nardetto ${ }^{7}$, Wolfgang Gieren ${ }^{8}$, and Grzegorz Pietrzynski ${ }^{9}$ \\ ${ }^{1}$ LESIA, Observatoire de Paris, Université PSL, CNRS, Sorbonne Université, Univ. Paris Diderot, Sorbonne Paris Cité, 5 place Jules \\ Janssen, 92195 Meudon, France \\ e-mail: pierre.kervella@obspm.fr \\ 2 European Southern Observatory, Alonso de Córdova 3107, Casilla 19001, Santiago, Chile \\ 3 Smithsonian Astrophysical Observatory, MS 4, 60 Garden Street, Cambridge, MA 02138, USA \\ 4 Konkoly Observatory, MTA CSFK, Konkoly Thege M. út 15-17, 1121, Hungary \\ 5 GEPI, Observatoire de Paris, Université PSL, CNRS, 5 Place Jules Janssen, 92190 Meudon, France \\ ${ }^{6}$ European Southern Observatory, Karl-Schwarzschild-Str. 2, 85748 Garching, Germany \\ 7 Université Côte d'Azur, OCA, CNRS, Lagrange, France \\ 8 Universidad de Concepción, Departamento de Astronomía, Casilla 160-C, Concepción, Chile \\ 9 Nicolaus Copernicus Astronomical Centre, Polish Academy of Sciences, Bartycka 18, 00-716 Warszawa, Poland
}

Received 8 September 2018 / Accepted 11 October 2018

\begin{abstract}
Context. The multiplicity of classical Cepheids (CCs) and RR Lyrae stars (RRLs) is still imperfectly known, particularly for RRLs. Aims. In order to complement the close-in short orbital period systems presented in Paper I, our aim is to detect the wide, spatially resolved companions of the targets of our reference samples of Galactic CCs and RRLs.

Methods. Angularly resolved common proper motion pairs were detected using a simple progressive selection algorithm to separate the most probable candidate companions from the unrelated field stars.

Results. We found 27 resolved, high probability gravitationally bound systems with CCs out of 456 examined stars, and one unbound star embedded in the circumstellar dusty nebula of the long-period Cepheid RS Pup. We found seven spatially resolved, probably bound systems with RRL primaries out of 789 investigated stars, and 22 additional candidate pairs. We report in particular new companions of three bright RRLs: OV And (companion of F4V spectral type), RR Leo (M0V), and SS Oct (K2V). In addition, we discovered resolved companions of 14 stars that were likely misclassified as RRLs.

Conclusions. The detection of resolved non-variable companions around CCs and RRLs facilitates the validation of their Gaia DR2 parallaxes. The possibility to conduct a detailed analysis of the resolved coeval companions of CCs and old population RRLs will also be valuable to progress on our understanding of their evolutionary path.
\end{abstract}

Key words. stars: variables: Cepheids - stars: variables: RR Lyrae - astrometry - proper motions - binaries: general binaries: visual

\section{Introduction}

Classical Cepheids (CCs) and RR Lyrae stars (RRLs) are essential standard candles for Galactic (e.g., Drake et al. 2013) and extragalactic (e.g., Riess et al. 2016) distance determinations. These pulsating stars are the subject of several studies following the first (Gaia Collaboration 2017; Iorio et al. 2018) and second (Clementini et al. 2019; Muraveva et al. 2018; Rimoldini et al. 2018) Gaia data releases. Their multiplicity fraction has a particular importance, as the presence of companions may bias their apparent brightness and affect their evolutionary path. The recent discovery of the binary evolution pulsators (Pietrzyński et al. 2012; Smolec et al. 2013) is an example of the potential impact of binarity on the properties of oscillating stars. Coeval, gravitationally bound companions are also valuable for conducting comparative evolutionary modeling.

\footnotetext{
$\star$ Tables A.1-C.1 are also available at the CDS via anonymous ftp to cdsarc.u-strasbg. fr $(130.79 .128 .5)$ or via http://cdsarc. u-strasbg.fr/viz-bin/qcat?J/A+A/623/A117
}

In Paper I (Kervella et al. 2019), we searched for companions of CCs and RRLs from the signature of the presence of a companion on their proper motion (PM). For this purpose, we used the positions measured by the HiPPARcos and Gaia spacecrafts at two epochs separated by $24.25 \mathrm{yr}$ to determine the PM of their center of mass. The presence of a companion results in a "virtual orbit" of the photocenter around the center of mass of the system (see, e.g., Benedict et al. 1999, 2000; Sahlmann et al. 2013). As the astrometric missions measured the position of the photocenter, we detected the presence of companions from a comparison of the two PM vectors (HIPParcos and Gaia DR2, hereafter GDR2) to the mean PM vector. The difference between the photocenter's PM vector and that of the center of mass is referred to as the proper motion anomaly (PMa) in the following. The time baseline of more than two decades between Hipparcos and Gaia provides a sensitivity to orbital periods of up to several hundred years, depending on the distance of the target and the mass ratio of the CC to its companion. However, its sensitivity decreases for longer orbital periods (i.e., millennia), as the 
principle of the determination of the mean PM of the center of mass (difference of positions) cancels the signature of the orbital $\mathrm{PM}$ of the photocenter if it is very slow.

In the present paper, we search for spatially resolved, common proper motion or gravitationally bound companions of our samples of CCs and RRLs. Despite the high contrast that makes their detection difficult, several spatially resolved CC companions have already been found using optical interferometry (Gallenne et al. 2013, 2014a, 2018a), adaptive optics (Gallenne et al. 2014b) or Hubble Space Telescope (HST) imaging (Evans et al. 2008, 2018a, 2016a). The database of the binary and multiple Galactic CCs maintained at Konkoly Observatory ${ }^{1}$ (Szabados 2003) provides a list of the known CCs in multiple systems. A database of candidate binaries with an RRL component is provided by Liška et al. $(2016 b)^{2}$. No RRL resolved companion is currently known, and only TU UMa has been convincingly demonstrated to be a binary and has accurate orbital parameters (Liška et al. 2016a; Paper I). Its companion is likely a white dwarf orbiting at a small angular separation $(\approx 10$ mas; Paper I).

In Sect. 3, we present the selection criteria that we adopted to discriminate the unrelated field stars from the physical companions. The resulting detections are presented in Sect. 5 for CCs (Sect. 5.1) and RRLs (Sect. 5.2). We also present the candidate companions of variable stars that were incorrectly classified as RRLs in Sect. 5.3. We discuss in Sect. 6 the results we obtained on selected individual stars, including the detections of PM anomalies presented in Paper I.

\section{Selected samples}

We adopted the list of 455 CCs from Berdnikov et al. (2000) plus Y Car, together with their listed photometric distances, that are based on multicolor period-luminosity relations. This catalog is tied to an LMC distance modulus of $\mu_{\mathrm{LMC}}=18.25$, that is too short compared to recent measurements. We therefore renormalized the listed distances using the distance modulus established by Pietrzyński et al. (2013). This correction increases the distances of all Cepheids in the Berdnikov et al. (2000) catalog by $\approx 11 \%$. The distance of Y Car is taken from Evans (1992a). The RRL sample was extracted from the General Catalogue of Variable Stars (GCVS; Samus et al. 2017) that comprises 8509 stars listed as RR type. We adopt for the RRLs the GDR2 parallaxes $\varpi_{\mathrm{G} 2}$. The catalog uncertainty of $\varpi_{\mathrm{G} 2}$ for the RRLs in the magnitude range of our sample is typically $30-100 \mu$ as. For the targets with $\varpi_{\mathrm{G} 2} \lesssim 0.5$ mas the uncertainty on the GDR2 parallaxes of the faint candidate companions becomes too large to enable an efficient selection process. We therefore limited our sample to the RRLs with a GDR2 parallax $\varpi_{\mathrm{GDR} 2}>0.5$ mas. This results in a sample of 789 stars classified as RRLs within $2 \mathrm{kpc}$.

The GDR2 parallaxes and PMs were corrected following the procedure described in Paper I, and we set a uniform uncertainty of $15 \%$ on all the adopted $456 \mathrm{CC}$ (photometric) and $789 \mathrm{RRL}$ (GDR2) parallaxes. It corresponds to the range of accepted parallaxes in the companion selection process (Sect. 3.1).

\section{Companion identification criteria}

We defined criteria for the selection of wide companion candidates based on (1) the similarity of their parallax, (2) their tangential differential velocity, (3) their projected linear separation.

\footnotetext{
1 http://www . konkoly.hu/CEP/intro.html

2 http://rrlyrbincan.physics.muni.cz
}

We also tested them to determine whether they are gravitationally bound.

\subsection{Parallax}

The GDR2 parallax is our primary criterion for the selection of potential companions. The first step in our selection is based on the compatibility of the GDR2 parallax of the field stars with the adopted value of the target stars (CCs or RRLs). The candidates whose GDR2 parallax is outside the range of expected values $( \pm 15 \%$ around the adopted parallax of the target) are rejected. We do not use a variable weight depending on the proximity in parallax; the candidate companions are uniformly rejected if they are outside the expected $\varpi$ uncertainty range. This choice is intended to reject the statistical outliers, reduce the number of false positive detections and ensure that we do not bias our detections toward the prior adopted value of the target parallax.

We also reject the field stars that have a parallax $S / N<3$ to reduce the number of inconclusive detections. The improvement of the uncertainties of the Gaia parallaxes in the future data releases will result in the inclusion of additional companion candidates.

\subsection{Proper motion}

We determined the difference in tangential velocity $d v_{\tan }$ (perpendicular to the line of sight) between the field stars and the tested CC and RRL. We excluded the candidates that exhibit a differential tangential velocity larger than $d v_{\tan , \max }=20 \mathrm{~km} \mathrm{~s}^{-1}$. This limit corresponds to an increase in projected separation of $\approx 20 \mathrm{pc}$ in ten million years. This is much larger than the usually considered limit for common proper motion pairs (see, e.g., Scholz 2016), even if Price-Whelan et al. (2017) report the existence of comoving pairs up to a separation of $10 \mathrm{pc}$. A field star is removed from the list of candidate companions if its $d v_{\tan }$ is more than $1 \sigma$ above $d v_{\tan \text {, max }}$. The candidates that exhibit a low differential velocity $d v_{\tan }<5 \mathrm{~km} \mathrm{~s}^{-1}$ are flagged as LowV.

We also set as a condition for field star selection that the position angle of its $\mathrm{PM}$ vector is within $\pm 15^{\circ}$ of that of the $\mathrm{PM}$ vector of the CC or RRL target star, if the candidate is located within a projected radius of $10 \mathrm{kau}$. This range of permitted angles accounts for a possible orbital motion. For wider separation candidates, the acceptance range for the PM vector position angle is reduced to $\pm 5^{\circ}$ to limit the number of false positives. When available, we adopted the mean proper motion $\mu_{\mathrm{HG}}$ computed from the HIPPARcos and GDR2 positions (see Paper I), to mitigate the effect of an orbiting close-in companion on the adopted PM vector. This is particularly important for the stars showing a strong PMa, for example the short-period Cepheid V1334 Cyg (Paper I; Gallenne et al. 2013, 2018a).

\subsection{Projected proximity}

The search radius around the CCs and RRLs is set to $1 \mathrm{pc}$ at the distance of each target, with a minimum angular radius of 1 arcmin. The probability that candidate companions are bound to the target star increases as their projected separation with the target becomes smaller. We therefore allocated a continuous linear weight in the ranking of the candidate companions to their linear projected distance to the target. The field stars that are within $50000 \mathrm{au}$ for CCs and $30000 \mathrm{au}$ from RRLs are flagged as Near. These different maximum radii take into account the difference in mass between CCs and RRLs, and also the fact that the young CCs can be found in open clusters (whose 
populations we wish to probe) contrary to old RRLs (except in the case of chance associations). These radii are comparable to the widest binary systems (Oh et al. 2017; Duchêne \& Kraus 2013; Kervella et al. 2017a), and they also incorporate the binaries possibly formed from adjacent prestellar cores with slow relative proper motion (Tokovinin 2017).

\subsection{Gravitationally bound candidates}

We test the possibility that the candidate companions are gravitationally bound to the target CC or RRL by comparing the differential tangential velocity $d v_{\tan }$ with the escape velocity $v_{\text {esc }}$ at their projected separation (see, e.g., Kervella et al. 2017a). This is a first-order approach as the third, radial component of the differential velocity is usually unknown for the field stars. It should be accounted for in a precise comparison of the relative velocity with the escape velocity. The expression of the escape velocity $v_{\text {esc }}$ for two bodies of total mass $m_{\text {tot }}$, located at a distance $r$ from each other is

$v_{\mathrm{esc}}=\sqrt{\frac{2 G m_{\mathrm{tot}}}{r}}$.

We adopt the predicted masses of the CC and RRL targets as described in Paper I. A limitation of this approach is that the true total mass $m_{\mathrm{tot}}$ of the systems is often higher than that of the $\mathrm{CC}$ or RRL alone. For instance, the system of $\delta$ Cep contains at least one close-in component of a mass of $\approx 1 M_{\odot}$ (Anderson et al. 2015; Gallenne et al. 2016), and a distant component HD 213307 (Benedict et al. 2002; Marengo et al. 2010) that is likely an earlytype, relatively massive binary (Sect. 6.1.9). The determination of the escape velocity for the different components of this quadruple system should be based on the total mass of the considered components that exceeds the mass of the Cepheid alone. To account for the mass of the secondary star (and possible additional components), we assumed an identical mass for the companion in the computation of the escape velocity by considering $m_{\text {tot }}=2 m_{\text {target }}$.

The candidate companions are flagged as Bound when they are already flagged as Near and their tangential differential velocity $d v_{\tan }$ is within $3 \sigma$ of the escape velocity $v_{\text {esc }}$ (they are also normally flagged as LowV). This relatively permissive criterion accounts for the significant uncertainties in the physical parameters used in the computation.

\section{Candidate companion parameters}

For CCs, individual color excess estimates $E(B-V)$ are available in the literature, and we adopted these values for their candidate companions. For the candidate companions of RRLs, we determined $E(B-V)$ from the 3D extinction maps by Green et al. (2018), Lallement et al. (2018), or Gontcharov (2017), depending on the sky coverage of each map. For the bright RR Lyrae stars RR Leo and SS Oct, we adopted the individual estimates by Feast et al. (2008).

For the candidates that have $J H K_{s}$ magnitudes from the 2MASS catalog (Cutri et al. 2003; Skrutskie et al. 2006), we derived their linear radius $R$ and effective temperature $T_{\text {eff }}$ from their broadband magnitudes using the visible-infrared surface brightness-color relations from Kervella et al. (2004) and the GDR2 parallax. For completeness, we also cross-identified the candidate companions in the WISE catalog (Wright et al. 2010; Cutri et al. 2012).

When $J H K_{s}$ infrared magnitudes were not available, we used Eq. (1) in Gaia Collaboration (2018) to compute the extinction in the $G, G_{\mathrm{BP}}$, and $G_{\mathrm{RP}}$ bands from the adopted value of the color excess $E(B-V)$. This provided the dereddened color excess $C_{\mathrm{XP}}=E\left(G_{\mathrm{BP}}-G_{\mathrm{RP}}\right)_{0}$ and the absolute dereddened $M_{G}$ magnitude of the candidates in the $G$ band. We then converted the dereddened color to an effective temperature using the polynomial expression by Jordi et al. (2010; their Eq. (2)):

$$
\log T_{\text {eff }}=3.999-0.654 C_{\mathrm{XP}}+0.709 C_{\mathrm{XP}}^{2}-0.316 C_{\mathrm{XP}}^{3} \text {. }
$$

For the stars with $C_{\mathrm{XP}}>1.4$, this polynomial expression is unreliable, and we adopt the following linear correction:

$$
\log T_{\text {eff,corr }}=\left(\frac{\log T_{\text {eff }}-3.3}{1.5-6}\right)\left(C_{\mathrm{XP}}-6\right)+3.3 .
$$

For main sequence candidate companions, we converted the effective temperature and absolute magnitude into spectral type using the grid by Pecaut $\&$ Mamajek $(2013)^{3}$ (see also Pecaut et al. 2012). In some cases, when the candidates are located close to the primary star, the derived $\left(T_{\text {eff }}, M_{G}\right)$ combination is inconsistent due to the contamination of the $G_{\mathrm{BP}}$ and $G_{\mathrm{RP}}$ magnitudes by the bright CCs or RRLs. The $G$ magnitude and astrometry can be correct while color photometry is contaminated, given the different sizes of the pixel windows: 18 pixels along scan for bright stars in the astrometric field ( $G$ band) against 60 pixels $\left(3.5^{\prime \prime}\right)$ for $G_{\mathrm{BP}}$ and $G_{\mathrm{RP}}$. When there was an inconsistency, we assumed that the candidate is a main sequence star and based our provisional spectral type determination on the absolute magnitude $M_{G}$ alone.

\section{Detected companions}

\subsection{Cepheid companions}

We individually examined the fields of the Bound or Near candidates by eye to check the dubious cases. The resulting list of candidates is presented in Table 1. We present in Figs. A.1A.5 the Second Generation Digitized Sky Survey Red (DSS2Red) fields around the CCs that show Bound candidate companions. Their properties and those of their companions are listed in Tables A.1 and A.2. Selected CCs with Near candidates are shown in Figs. A.6 and A.7. The full list of detected Near candidate companions of CCs is given in Tables A.1 and A.2.

For the more distant CCs of our sample, the probability of chance associations for the detected candidate companions is increased compared to the closer CCs and the RRLs (which are on average located at shorter distances than CCs). Figure 1 shows the histogram of the CCs with detected Near and Bound candidates. The decrease in the sensitivity of the algorithm with distance is clearly visible in the right panel. We observe a peak fraction of $\approx 10 \%$ of CCs with Bound candidate companions. A validation of the candidate companions for the distant CCs will be possible using the future Gaia data releases, which will provide more accurate estimates of their relative parallaxes and tangential velocities. The measurement of radial velocities for the CCs and their candidate companions will provide their full $3 \mathrm{D}$ relative velocity and therefore enable a stringent test of their gravitational bind.

\subsection{RR Lyrae star companions}

RR Lyrae stars are old population stars, with a typical age of $10 \mathrm{Ga}$, that usually belong to the thick disk of the Galaxy. On average, nearby RRLs have significantly faster proper motions

\footnotetext{
3 http://www . pas.rochester.edu/ emamajek/EEM_dwarf_ UBVIJHK_colors_Teff.txt
} 
Table 1. Wide companions of Cepheids for the targets with Near (N) or Bound (B) resolved candidate companions.

\begin{tabular}{|c|c|c|c|c|c|c|c|}
\hline Target & $\begin{array}{c}\varpi_{\text {exp. }} \\
\text { (mas) }\end{array}$ & $\begin{array}{r}\text { Period } \\
\text { (d) }\end{array}$ & Total & $\mathrm{N}$ & B & Vis. & Comment \\
\hline \multicolumn{8}{|c|}{ Cepheids with Bound candidates } \\
\hline TV CMa & 0.477 & 4.67 & 4 & 1 & 1 & $\checkmark$ & Tight, high probability bound companion \\
\hline ER Car & 0.959 & 7.72 & 41 & 2 & 1 & $\checkmark$ & Very wide comoving candidate \\
\hline CECas B & 0.343 & 4.48 & 15 & 1 & 1 & $\checkmark$ & Known physical Cepheid companion (CE Cas A) \\
\hline DFCas & 0.462 & 3.83 & 7 & 1 & 1 & $\checkmark$ & Slow PM, possible association \\
\hline V0659 Cen & 1.287 & 5.62 & 66 & 1 & 1 & $\checkmark$ & Very wide comoving candidate \\
\hline delta Cep & 3.755 & 5.37 & 53 & 1 & 1 & $\checkmark$ & Known physical companion $(\delta$ Cep B) \\
\hline AXCir & 1.917 & 5.27 & 79 & 2 & 1 & $\checkmark$ & Wide, probably bound companion \\
\hline BP Cir & 1.700 & 2.40 & 101 & 2 & 1 & $\checkmark$ & Very wide comoving companion, possible group \\
\hline $\mathrm{R}$ Cru & 1.170 & 5.83 & 49 & 1 & 1 & $\checkmark$ & Tight, probably bound companion \\
\hline X Cru & 0.678 & 6.22 & 36 & 2 & 1 & $\checkmark$ & Wide, possibly bound companion \\
\hline VW Cru & 0.710 & 5.27 & 22 & 1 & 1 & $\checkmark$ & Possible comoving group \\
\hline V0532 Cyg & 0.727 & 4.68 & 19 & 1 & 1 & $\checkmark$ & Wide, probably bound companion \\
\hline V1046 Cyg & 0.372 & 4.94 & 6 & 1 & 1 & $\checkmark$ & Tight, high probability bound companion \\
\hline CV Mon & 0.601 & 5.38 & 27 & 3 & 2 & $\checkmark$ & High probability bound candidates \\
\hline RS Nor & 0.487 & 6.20 & 36 & 2 & 1 & $\checkmark$ & Tight, probably bound companion \\
\hline SY Nor & 0.429 & 12.65 & 14 & 2 & 1 & $\checkmark$ & Tight, probably bound companion \\
\hline QZ Nor & 0.556 & 5.41 & 69 & 3 & 1 & $\checkmark$ & Wide, possible chance association \\
\hline AW Per & 1.218 & 6.46 & 10 & 1 & 1 & $\checkmark$ & Tight bound companion \\
\hline USgr & 1.669 & 6.75 & 165 & 3 & 2 & $\checkmark$ & Very wide, possible comoving cluster \\
\hline V0350 Sgr & 1.141 & 5.15 & 57 & 2 & 1 & $\checkmark$ & High probability bound companion \\
\hline V0950 Sco & 1.073 & 4.82 & 92 & 2 & 1 & $\checkmark$ & Likely bound candidate \\
\hline CM Sct & 0.518 & 3.92 & 21 & 1 & 1 & $\checkmark$ & Wide comoving association \\
\hline EV Sct & 0.556 & 4.40 & 43 & 1 & 1 & $\checkmark$ & Wide, probably bound candidate, possible group \\
\hline Polaris ( $\alpha \mathrm{UMi})$ & 7.540 & 5.67 & 15 & 1 & 1 & $\checkmark$ & Known physical companion (Polaris B) \\
\hline SX Vel & 0.490 & 9.55 & 17 & 1 & 1 & $\checkmark$ & Tight, high probability bound companion \\
\hline CS Vel & 0.292 & 5.90 & 10 & 1 & 1 & $\checkmark$ & Possibly bound candidate and comoving group \\
\hline DK Vel & 0.431 & 2.48 & 17 & 1 & 1 & $\checkmark$ & Wide, possibly bound candidate \\
\hline \multicolumn{8}{|c|}{ Cepheids with Near candidates } \\
\hline FF Aql & 2.048 & 6.40 & 60 & 1 & 0 & - & PM divergence, unlikely association \\
\hline V0916 Aql & 0.325 & 13.44 & 3 & 1 & 0 & $\checkmark$ & Tight companion, small parallax \\
\hline eta Aql & 3.755 & 7.18 & 33 & 1 & 0 & - & PM divergence, unlikely association \\
\hline CK Cam & 1.959 & 3.29 & 27 & 1 & 0 & - & PM divergence, unlikely association \\
\hline Y Car & 0.695 & 3.64 & 32 & 1 & 0 & $\checkmark$ & Comoving group, possible cluster \\
\hline UX Car & 0.710 & 3.68 & 29 & 1 & 0 & - & Very wide, unlikely association \\
\hline UZ Car & 0.437 & 5.20 & 30 & 1 & 0 & - & Very wide, PM divergence, unlikely association \\
\hline XY Car & 0.366 & 12.43 & 9 & 1 & 0 & - & Very wide, possible comoving star \\
\hline EY Car & 0.446 & 2.88 & 27 & 1 & 0 & - & Tangential velocity difference, possible association \\
\hline DDCas & 0.322 & 9.81 & 3 & 1 & 0 & - & Tangential velocity difference, unlikely association \\
\hline VW Cen & 0.256 & 15.04 & 5 & 1 & 0 & $\checkmark$ & Very wide, possibly comoving companion \\
\hline XX Cen & 0.589 & 10.95 & 23 & 1 & 0 & - & PM difference, unlikely association \\
\hline AY Cen & 0.639 & 5.31 & 45 & 1 & 0 & $\checkmark$ & Slight PM divergence, possible association \\
\hline AV Cir & 1.802 & 3.07 & 57 & 1 & 0 & - & Very wide, unrelated field stars \\
\hline T Cru & 1.306 & 6.73 & 45 & 1 & 0 & - & Wide, PM divergence, unlikely association \\
\hline SU Cyg & 1.234 & 3.85 & 74 & 1 & 0 & $\checkmark$ & Probably comoving unbound companion \\
\hline SZ Cyg & 0.419 & 15.11 & 5 & 1 & 0 & $\checkmark$ & Possible comoving companion \\
\hline V1334 Cyg & 1.502 & 4.75 & 24 & 1 & 0 & $\checkmark$ & Probable comoving companion \\
\hline RR Lac & 0.515 & 6.42 & 12 & 1 & 0 & - & Wide, unlikely association \\
\hline S Nor & 1.126 & 9.75 & 104 & 2 & 0 & $\checkmark$ & Very wide, one possibly bound and one unbound \\
\hline U Nor & 0.699 & 12.64 & 35 & 1 & 0 & - & Wide, unlikely association \\
\hline Y Oph & 1.186 & 17.12 & 20 & 1 & 0 & $\checkmark$ & Very wide, possibly comoving candidate \\
\hline AQ Pup & 0.312 & 30.10 & 6 & 1 & 0 & $\checkmark$ & Possible comoving companion \\
\hline Y Sgr & 2.146 & 5.77 & 90 & 2 & 0 & $\checkmark$ & Wide, possible association \\
\hline XX Sgr & 0.733 & 6.42 & 39 & 1 & 0 & - & Tangential velocity difference, unlikely association \\
\hline APSgr & 1.287 & 5.06 & 91 & 2 & 0 & $\checkmark$ & Wide, possibly comoving candidate \\
\hline Y Sct & 0.581 & 10.34 & 18 & 1 & 0 & - & Wide, unlikely association \\
\hline $\mathrm{R}$ TrA & 1.733 & 3.39 & 66 & 1 & 0 & $\checkmark$ & Wide, uncertain companion, slight PM offset \\
\hline LR TrA & 1.048 & 3.44 & 40 & 1 & 0 & - & Wide, unlikely association, PM difference \\
\hline
\end{tabular}

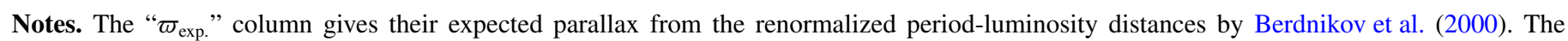
"Total" column gives the number of GDR2 field stars with compatible parallaxes that were examined as candidates. The "Vis" column indicates the result of a visual inspection of the field of the considered targets, with $\checkmark$ indicating a likely comoving system and - a dubious association. The observational properties and field charts of the Cepheids and their associated candidate companions are presented in Appendix A. 
than the surrounding field stars, making their common proper motion companions easier to identify. We applied our companion search criteria to 789 RRLs, and this resulted in 7 targets with candidates flagged as Bound candidates and 22 flagged as Near. The 7 RRLs with Bound candidates are listed in Table 2, and their observational properties are given in Table B.1. The full list of detected Near candidate companions of RRLs is given in Tables B.2 and B.3. The histogram of the RRLs and various variable stars with detected candidate companions is presented in Fig. 2. For the nearby stars, we detect Bound candidate companions around $\approx 5-10 \%$ of the examined targets. The fields surrounding the RRLs with Bound candidates are shown in Figs. B.1 and B.2, and a selection of the RRLs with Near candidates is shown in Fig. B.2.

\subsection{Variables of other classes}

Fourteen targets that were incorrectly classified as RRLs have Bound candidate companions. The results of the visual inspection of the corresponding fields are given in Table 2 and the detailed candidate properties are listed in Table C.1. Their respective field charts are presented in Figs. C.1-C.3.

\section{Notes on individual stars}

In this section we present a selection of individual stars from our sample that host highly probable resolved physical companions. We also discuss the stars for which a significant PMa was identified in Paper I.

\subsection{Cepheids}

\subsubsection{U Aql}

The quest for masses and luminosities for CCs has benefitted greatly from the availability of the ultraviolet spectrum using the International Ultraviolet Explorer (IUE) and HST telescopes. Through these studies, the picture of multiplicity has become increasingly complex. The Cepheid U Aql ( $P=7.02 \mathrm{~d}$; HD 183344) is a good case in point. Its substantial orbital motion was only recognized in 1979 (Slovak et al. 1979). The discussion of Welch et al. (1987) provides an orbit and a summary of previous velocity information. The spectrum of the hottest star in the system dominates in the ultraviolet below about $\lambda=200 \mathrm{~nm}$, and IUE observations have been discussed by Bohm-Vitense \& Proffitt (1985) and Evans (1992b). These studies provide a temperature of $9300 \pm 100 \mathrm{~K}$ and a spectral type of B9.8V, respectively.

We detect a very strong PMa on U Aql. From its combination with the spectroscopic orbital parameters by Gallenne et al. (2018b), we determined in Paper I that its companion has a mass of $1.9 \pm 0.3 M_{\odot}$ and is orbiting on a $5.6 \mathrm{au}$ orbit. This mass is slightly lower than expected from the effective temperature of $T_{\text {eff }}=9300 \pm 100 \mathrm{~K}$ determined from IUE spectroscopy by Bohm-Vitense \& Proffitt (1985) and the spectral type of B9.8V from Evans (1992b). We do not detect any resolved companion of U Aql.

\subsubsection{FF Aql}

FF Aql is a known binary system whose spectroscopic orbital parameters were determined by Evans et al. (1990). The astrometric observations by Benedict et al. (2007) using the HST Fine Guidance Sensor (FGS) revealed the orbital shift of the photo-
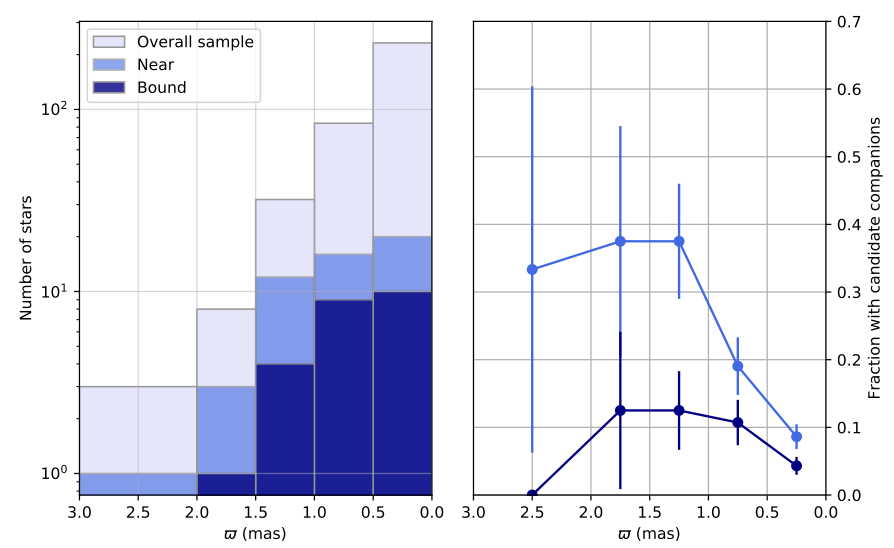

Fig. 1. Left panel: histogram of the CCs with candidate Near and Bound companions as a function of parallax. Right panel: fraction of the observed CCs with candidate companions. The error bars represent the binomial proportion $68 \%$ confidence interval.
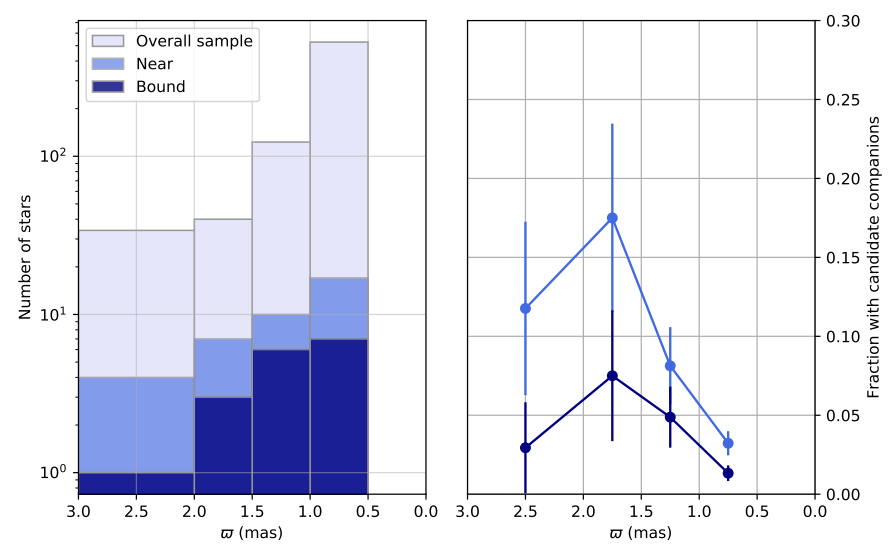

Fig. 2. Left panel: histogram of the RRLs and various variables with candidate Near and Bound companions as a function of parallax. The lower parallax limit is set at $\varpi=0.5$ mas for our RRL sample. Right panel: fraction of stars with candidate companions. The error bars represent the binomial proportion $68 \%$ confidence interval.

center of the system. In Paper I, we identified a strong PMa on FF Aql with a S/N from the GDR2 measurement of $\Delta_{\mathrm{G} 2}=5.6$. Combining the PM anomalies with the spectroscopic orbital parameters by Gallenne et al. (2018b), we derived the orbital parameters of the system and the mass of the secondary component $\left(m_{2} \approx 0.8 \pm 0.1 M_{\odot}\right.$; Paper I), which is lower than the estimate of $1.5 M_{\odot}$ by Evans et al. (1990) from IUE spectroscopy. Future Gaia data releases will provide an accurate determination of the parameters of the photocenter astrometric orbit, as the orbital period of the system $(P=1433 \mathrm{~d})$ is a good match to the observing lifetime of the satellite.

Gallenne et al. (2014b) searched for resolved companions of the short-period Cepheid FF Aql using adaptive optics up to a separation of $1.7^{\prime \prime}$, but did not find any. Gallenne et al. (2018b) detected a possible close-in companion using near-infrared interferometry. The visual companion found by Jeffers et al. (1963) and recovered by Roberts (2011) and Evans et al. (2016a) is Gaia DR2 4514145288240592512 at a separation of $6.98^{\prime \prime}$ from the CC. We find that its GDR2 parallax $(\varpi=1.971 \pm 0.041$ mas $)$ is compatible with that of FF Aql $(\varpi=1.839 \pm 0.107$ mas $)$. However, its PM vector $\left(\mu=[-8.52 \pm 0.07,-19.93 \pm 0.07] \mathrm{mas} \mathrm{a}^{-1}\right)$ is very different from the mean HipPARcos-Gaia PM vector of the CC $\left(\mu_{\mathrm{HG}}=\right.$ 
$[-0.14 \pm 0.01,-9.34 \pm 0.01]$ mas $\left.^{-1}\right)$. This corresponds to a projected relative velocity of $\approx 30 \mathrm{~km} \mathrm{~s}^{-1}$. This in principle excludes that the stars are gravitationally bound, confirming the conclusion by Evans et al. (1990). However, the coincidence in terms of position and parallax is quite remarkable from a statistical point of view. This candidate could still be comoving with the $\mathrm{CC}$, and may be bound, if it is a close binary and its measured $\mathrm{PM}$ is affected by its orbital motion.

\subsubsection{RW Cam}

RW Cam exhibits a strong PMa signal-to-noise ratio $\left(\Delta_{\mathrm{G} 2}=\right.$ 9.3) (Paper I). It is a long-period CC $(P=16.41 \mathrm{~d})$ that is a known binary (Madore 1977; Bohm-Vitense \& Proffitt 1985; Evans 1994). As reported in Paper I, we estimate that its orbital period is probably on the order of a few hundred years, for a semimajor axis of $\approx 200$ mas. We do not detect any additional resolved companion of RW Cam.

\subsubsection{Y Car}

Bohm-Vitense et al. (1997) found that the spectral type of the companion of Y Car is B9V, and Evans et al. (2005) showed that Y Car is actually a triple system. This companion was detected by Gallenne et al. (2018b) via near-infrared interferometry. We reported in Paper I a very strong PMa S/N $\left(\Delta_{\mathrm{G} 2}=14.8\right)$ that confirms the multiplicity of this short-period double-mode Cepheid $(P=3.64 \mathrm{~d}$ for the fundamental mode $)$. We did not detect any Bound candidate companion of Y Car, but we identified several nearby stars with comparable PM vectors that could constitute a comoving group (Fig. A.6).

\subsubsection{YZ Car}

The combination of the spectroscopic orbital elements of Gallenne et al. (2018b; which are in agreement with those determined by Anderson et al. 2016) and the PMa vectors results in a companion mass of $m_{2} \approx 1.9 \pm 0.3 M_{\odot}$ (Paper I) for the companion of the long-period pulsator YZ Car $(P=18.2 \mathrm{~d})$. The interferometric companion search by Gallenne et al. (2018b) did not reveal the faint secondary, but established an upper limit of B3V on its spectral type, in agreement with the estimate of B8V-A0V from Evans \& Butler (1993) and the mass derived in Paper I, which corresponds to an A-type dwarf.

We did not detect any Bound candidate companions, but two nearby stars present comparable proper motions and parallaxes (Fig. A.6).

\subsubsection{CE Cas $A B$}

This visual binary is composed of two CCs, labeled components A $(P=5.14 \mathrm{~d})$ and $\mathrm{B}(P=4.48 \mathrm{~d})$. This is the only such configuration known in the Milky Way. The projected separation of the two stars is $7.2 \mathrm{kau}$, corresponding to an orbital period on the order of 5000 years. CE Cas A and B are members of the open cluster NGC 7790 (Majaess et al. 2013), together with the Cepheid CF Cas $(P=4.875 \mathrm{~d})$. The two components of CE Cas are present in the GDR2, but with statistically different parallaxes $\left(\varpi_{A}=0.317 \pm 0.031 \mathrm{mas} ; \varpi_{B}=0.262 \pm 0.030 \mathrm{mas}\right)$. From their PM vectors, we confirm, however, that the two Cepheids are gravitationally bound (Fig. A.1). The GDR2 parallax of component $\mathrm{B}$ is likely biased, possibly due to light contamination from the nearby component $\mathrm{A}$. We did not find any additional resolved candidate companions of CE Cas.

\subsubsection{SU Cas}

The short-period pulsator SU Cas $(P=1.95 \mathrm{~d})$ is a member of a cluster of young stellar objects (Majaess et al. 2012a), and its distance $(d=414 \pm 11 \mathrm{pc} ; \varpi=2.42 \pm 0.06$ mas $)$ was estimated by Majaess et al. (2012b). This value is compatible with the GDR2 parallax $(\varpi=2.15 \pm 0.08$ mas $)$. SU Cas is located close to a reflection nebula (Turner \& Evans 1984; Magakian 2003). It exhibits a strong PMa with a $\mathrm{S} / \mathrm{N}$ of $\Delta_{\mathrm{G} 2}=5.6$ (Paper I). The combination of the spectroscopic orbital parameters determined by Groenewegen (2008) and the PM anomalies point to the presence of a very low mass companion on a tight orbit of only $1.7 \mathrm{au}$. This companion is different from the $2.4 M_{\odot}$ candidate with spectral type B9.5V spectral type listed by Evans et al. (2013) that orbits the Cepheid at a much larger distance of $\approx 100 \mathrm{au}$. No detection is reported by Gallenne et al. (2015) from near-infrared interferometric observations, setting an upper limit of A0 to the spectral type of close-in companions. We did not detect any Bound resolved stellar companion of SU Cas in the GDR2.

\subsubsection{V0659 Cen}

Evans et al. (2013) identified a close companion of the shortperiod Cepheid V0659 Cen $(P=5.62 \mathrm{~d})$ at a separation of $0.63^{\prime \prime}$ using HST/WFC3 imaging. We found a marginal PMa at the HipPaRcos epoch $\left(\Delta_{\text {Hip }}=2.7\right)$, but it is not significant at the $\operatorname{GDR} 2$ epoch $\left(\Delta_{\mathrm{G} 2}=1.6\right)$. We note, however, that the error bars of the GDR2 PM vector are larger than usual, possibly due to the presence of the close companion at a separation of $0.63^{\prime \prime}$ that is not listed separately in the GDR2 catalog.

We found a resolved common proper motion candidate star of spectral type M3V at a very wide projected separation of $48 \mathrm{kau}$ (Fig. A.1) that does not correspond to the candidates observed by Evans et al. (2016a; see also Evans et al. 2016b).

\subsection{9. $\delta$ Cep}

As shown by Majaess et al. (2012c), the prototype Cepheid $\delta$ Cep is a member of a cluster that also includes the K1.5b supergiant $\zeta$ Cep. This Cepheid has shown a periodic X-ray variability (Engle et al. 2017) and evidence of a significant mass loss (Matthews et al. 2012). Figure 3 shows the field around $\delta \mathrm{Cep}$, with some of the stars in the cluster that show a comparable proper motion.

The bright visual companion $\delta$ Cep B (HD 213307) is associated with its surrounding nebula (Marengo et al. 2010; Matthews et al. 2012) as confirmed by the presence of a bow shock. The GDR2 parallax of $\delta$ Cep B $\left(\varpi_{\mathrm{G} 2, \mathrm{~B}}=3.393 \pm 0.049\right.$ mas $)$ is noticeably smaller than the HST FGS determination by Benedict et al. (2002) $\left(\varpi_{\mathrm{FGS}}=\right.$ $3.66 \pm 0.15$ mas $)$, as well as the Hipparcos parallax $\left(\varpi_{\text {Hip }}=\right.$ $3.77 \pm 0.16 \mathrm{mas}$ ) and the combined cluster distance from Majaess et al. (2012c) $\left(\varpi_{\text {comb }}=3.68 \pm 0.08\right.$ mas $)$. The GDR2 parallax of $\delta$ Cep B is consistent with the distance derived by Borgniet et al. (2018) using the HR-SPIPS spectral analysis technique. Rescaling the SPIPS fit of Mérand et al. (2015), this larger distance corresponds to a spectroscopic projection factor of $p=1.39 \pm 0.03$. The spectral type of $\delta \mathrm{Cep} \mathrm{B}$ was estimated in the B7-B8 III-IV range by Benedict et al. (2002), with a probable F0V companion. This corresponds to a mass of $\approx 5.6 M_{\odot}$ for the pair $\mathrm{Ba}+\mathrm{Bb}$. The PMa vector of $\delta \mathrm{Cep} \mathrm{B}$ exhibits $\mathrm{S} / \mathrm{N}$ levels of $\Delta_{\mathrm{Hip}}=3.8$ and $\Delta_{\mathrm{G} 2}=2.1$, respectively for HIPPARCos and GDR2 (Table 3), confirming its binarity. Its differential tangential 
P. Kervella et al.: Multiplicity of Galactic Cepheids and RR Lyrae stars from Gaia DR2. II.

Table 2. RR Lyrae stars and other types of variable stars with Near (N) or Bound (B) resolved candidate companions.

\begin{tabular}{|c|c|c|c|c|c|c|c|}
\hline Target & $\begin{array}{r}\varpi_{\mathrm{G} 2} \\
(\mathrm{mas})\end{array}$ & $\begin{array}{l}\text { Period } \\
\text { (d) }\end{array}$ & Total & $\mathrm{N}$ & B & Vis. & Comment \\
\hline \multicolumn{8}{|c|}{ RR Lyrae with Bound candidates } \\
\hline OV And & 0.938 & 0.471 & 9 & 1 & 1 & $\checkmark$ & Tight, high probability bound companion \\
\hline CS Del & 0.635 & 0.366 & 7 & 1 & 1 & $\checkmark$ & Very tight, high probability bound companion \\
\hline V0893 Her & 2.679 & 0.492 & 13 & 1 & 1 & $\checkmark$ & High probability bound, slight PM difference \\
\hline RR Leo & 1.032 & 0.452 & 1 & 1 & 1 & $\checkmark$ & Tight, high probability bound system \\
\hline SS Oct & 0.862 & $0.622 / 0$ & 3 & 1 & 1 & $\checkmark$ & Very tight bound companion \\
\hline EY Oph & 1.850 & NA & 36 & 2 & 1 & $\checkmark$ & Slight PM divergence, possibly bound companion \\
\hline V0487 Sco & 1.069 & 0.329 & 59 & 1 & 1 & $\checkmark$ & Fast PM, probably bound companion \\
\hline \multicolumn{8}{|c|}{ RR Lyrae with Near candidates } \\
\hline V0830 Cyg & 0.618 & $0.401 / 0$ & 27 & 1 & 0 & - & Probable chance association \\
\hline $\mathrm{CZ} \mathrm{Lac}$ & 0.852 & $0.432 / 0$ & 25 & 1 & 0 & $\checkmark$ & PMa, probably bound, triple system \\
\hline V0424 Lyr & 1.579 & $0.580 / 0$ & 33 & 1 & 0 & - & Rich star field, probable chance association \\
\hline AG Nor & 0.978 & 0.505 & 68 & 3 & 0 & $\checkmark$ & Close parallax value, possibly comoving \\
\hline KP Nor & 0.520 & NA & 25 & 1 & 0 & - & PM difference, chance association \\
\hline IT Oph & 6.206 & NA & 578 & 1 & 0 & - & Dense star field, chance association \\
\hline MS Oph & 0.658 & NA & 26 & 1 & 0 & - & Probable chance association \\
\hline V1693 Oph & 0.677 & 0.522 & 27 & 1 & 0 & - & Uncertain association \\
\hline UY Ori & 2.840 & NA & 18 & 1 & 0 & - & Divergent PM vectors \\
\hline V1154 Ori & 1.087 & NA & 51 & 1 & 0 & $\checkmark$ & Probable comoving association \\
\hline V0701 Sgr & 2.000 & 0.627 & 127 & 2 & 0 & - & PM difference, chance association \\
\hline V2481 Sgr & 0.910 & NA & 105 & 1 & 0 & - & Divergent PM, chance association \\
\hline V2626 Sgr & 1.363 & 0.462 & 88 & 1 & 0 & $\checkmark$ & Slight PM position angle difference, uncertain \\
\hline V3531 Sgr & 0.765 & 0.542 & 26 & 1 & 0 & - & Very wide, unlikely association \\
\hline V4107 Sgr & 1.265 & NA & 224 & 1 & 0 & - & Dense stellar field, association unclear \\
\hline V4313 Sgr & 1.700 & NA & 279 & 2 & 0 & $\checkmark$ & Possible comoving group \\
\hline V4355 Sgr & 2.433 & NA & 361 & 5 & 0 & - & Probable chance association \\
\hline V4591 Sgr & 1.837 & NA & 276 & 1 & 0 & - & PM difference, chance association \\
\hline IY Sco & 2.530 & NA & 309 & 2 & 0 & - & Rich star field, chance association \\
\hline KN Sco & 0.959 & NA & 43 & 1 & 0 & - & Unlikely association \\
\hline V0828 Sco & 0.877 & NA & 33 & 1 & 0 & - & PM difference, chance association \\
\hline V0348 Sct & 1.249 & NA & 45 & 1 & 0 & - & Chance association \\
\hline \multicolumn{8}{|c|}{ Other variable types with Bound candidates } \\
\hline HM Aql & 1.829 & 0.345 & 28 & 1 & 1 & $\checkmark$ & Relatively wide companion, likely bound \\
\hline $\mathrm{EN} \mathrm{CMi}$ & 0.569 & 0.540 & 8 & 1 & 1 & $\checkmark$ & Wide, high probability bound candidate \\
\hline NQ Cyg & 0.938 & 0.312 & 25 & 1 & 1 & $\checkmark$ & Eclipsing binary, fast PM, probably bound companion \\
\hline V1391 Cyg & 1.232 & 0.596 & 17 & 1 & 1 & $\checkmark$ & Eclipsing binary, tight companion \\
\hline V2121 Cyg & 25.597 & $0.800^{a}$ & 3 & 1 & 1 & $\checkmark$ & $\gamma$ Dor pulsator, PMa, fast proper motion \\
\hline UU Dor & 0.856 & NA & 5 & 1 & 1 & $\checkmark$ & Eclipsing binary, tight high probability bound companion \\
\hline IW Lib & 3.571 & $1.783^{b}$ & 22 & 1 & 1 & $\checkmark$ & W UMa eclipsing, high probability bound companion \\
\hline AZ Men & 1.088 & 0.318 & 8 & 1 & 1 & $\checkmark$ & High probability bound companion \\
\hline V1171 Oph & 1.099 & NA & 16 & 2 & 1 & $\checkmark$ & Uncertain variable class, high probability bound companion \\
\hline V1330 Sgr & 1.229 & 0.427 & 193 & 2 & 1 & - & Wide, uncertain companion \\
\hline V1382 Sgr & 1.611 & 0.493 & 316 & 1 & 1 & $\checkmark$ & Comoving candidate, slight PM difference \\
\hline V2248 Sgr & 1.948 & 0.315 & 22 & 1 & 1 & $\checkmark$ & W UMa eclipsing, high probability bound companion \\
\hline V3166 Sgr & 6.646 & NA & 192 & 3 & 2 & $\checkmark$ & Uncertain variable class, two high probability bound \\
\hline HR Sco & 7.370 & NA & 425 & 2 & 1 & - & Uncertain variable class, dense field, likely chance association \\
\hline
\end{tabular}

Notes. A mention of " 0 " after the period indicates a fundamental mode pulsator as identified in the GDR2 catalog (rrlyrae table). The observational properties and field charts of the RRLs and their associated candidate companions are presented in Appendix B.

References. ${ }^{(a)}$ Cuypers et al. (2009); ${ }^{(b)}$ Pojmanski (2002).

velocity with respect to $\mathrm{A}$ is $d v_{\tan }=1.18 \pm 0.03 \mathrm{~km} \mathrm{~s}^{-1}$ (Table 3 ). This is comparable to the escape velocity at a separation of $12 \mathrm{kau}$, i.e., $v_{\mathrm{esc}} \approx 0.90 \mathrm{~km} \mathrm{~s}^{-1}$ for a total system mass of $11 M_{\odot}$ (for the four components of the system, see below). Measuring the radial velocity of $\delta$ Cep $\mathrm{B}$ is unfortunately a difficult task as it is a fast rotating $\operatorname{star}\left(v_{\mathrm{rot}} \sin i \approx 140 \mathrm{~km} \mathrm{~s}^{-1}\right.$; Bernacca \& Perinotto 1970). Moreover, the presence of the F0V companion $\delta \mathrm{Cep} \mathrm{Bb}$ with an estimated orbital period of $390 \mathrm{~d}$ and an induced orbital velocity amplitude of $K_{1} \approx 15 \mathrm{~km} \mathrm{~s}^{-1}$ (Benedict et al. 2002) also complicates the measurement of the $\gamma$-velocity of $\mathrm{Ba}+\mathrm{Bb}$ (i.e., its center-of-mass radial velocity). So at the moment, we cannot firmly conclude that $\delta$ Cep B is gravitationally bound to A. However, the low differential tangential velocity of the two objects, their proximity in space, and their high masses are statistically strong indications that they are gravitationally bound.

A variation in the $\gamma$-velocity of $\delta$ Cep A reported by Anderson et al. (2015) revealed the presence of the close-in orbiting companion $\delta$ Cep Ab. $\delta$ Cep exhibits a strong PMa in the HiPPARcos and GDR2 catalogs, at S/N levels of $\Delta_{\text {Hip }}=7.0$ and 

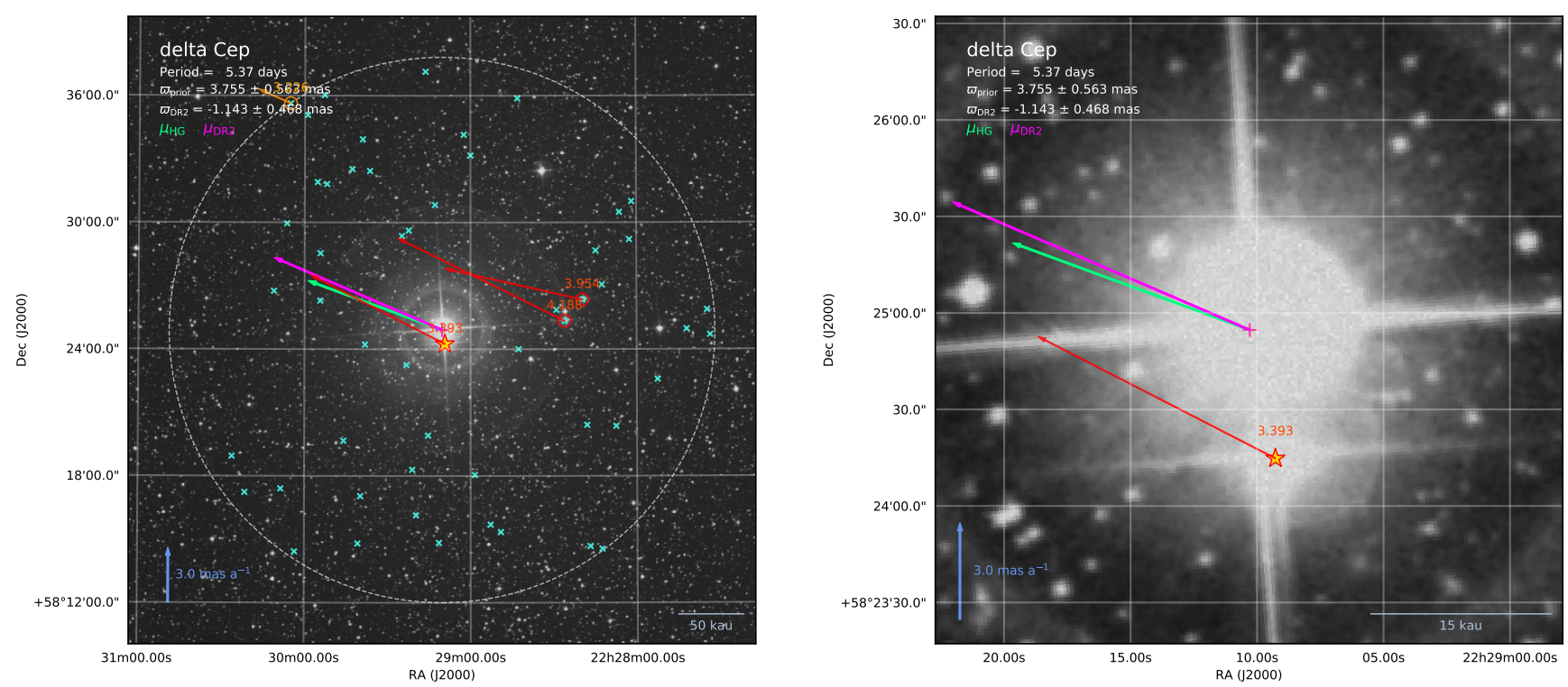

Fig. 3. Field around $\delta$ Cep (left panel: wide field; right panel: narrow field), with its companion $\delta$ Cep B (yellow star) and other comoving stars. The background image is from the DSS2-Red.

Table 3. Absolute and relative motion of $\delta$ Cep A and $\delta$ Cep B (HD 213307).

\begin{tabular}{lcccc}
\hline & \multicolumn{2}{c}{$\delta$ Cep A } & \multicolumn{2}{c}{$\delta$ Cep B } \\
\cline { 2 - 5 } & \multicolumn{1}{c}{$\alpha$} & $\delta$ & $\alpha$ & $\delta$ \\
\hline $\boldsymbol{\mu}_{\mathrm{HG}}$ & $+14.069_{0.009}$ & $+2.703_{0.016}$ & $+14.134_{0.009}$ & $+3.548_{0.015}$ \\
$d v_{\mathrm{tan}}$ & & & $+0.089_{0.013}$ & $+1.181_{0.021}$ \\
\hline $\boldsymbol{\mu}_{\mathrm{H}}$ & $+15.35_{0.22}$ & $+3.52_{0.18}$ & $+16.19_{0.59}$ & $+4.28_{0.50}$ \\
$\Delta \boldsymbol{\mu}_{\mathrm{H}}$ & $+1.28_{0.23}$ & $+0.82_{0.19}$ & $+2.06_{0.59}$ & $+0.73_{0.51}$ \\
$\Delta_{\mathrm{H}}$ & \multicolumn{2}{c}{7.0} & \multicolumn{2}{c}{3.8} \\
\hline $\boldsymbol{\mu}_{\mathrm{G} 2}$ & $+17.64_{0.81}$ & $+3.98_{0.73}$ & $+14.09_{0.09}$ & $+3.79_{0.09}$ \\
$\Delta \boldsymbol{\mu}_{\mathrm{G} 2}$ & $+3.57_{0.82}$ & $+1.27_{0.72}$ & $-0.04_{0.11}$ & $+0.24_{0.12}$ \\
$\Delta_{\mathrm{G} 2}$ & \multicolumn{2}{c}{4.7} & \multicolumn{3}{c}{2.1} \\
\hline
\end{tabular}

Notes. The absolute and linear proper motions $(\mu$ and $\Delta \mu)$ are expressed in mas $\mathrm{a}^{-1}$ and the differential tangential velocity of $\mathrm{B}$ relative to $\mathrm{A}$ $\left(d v_{\tan }\right)$ in $\mathrm{km} \mathrm{s}^{-1}$. The angular proper motion was converted to velocity using the GDR2 parallax of component $\mathrm{B}\left(\varpi_{\mathrm{G} 2, \mathrm{~B}}=3.393 \pm 0.049\right.$ mas $)$.

$\Delta_{\mathrm{G} 2}=4.7$ (see Table 3 and Paper I), respectively. Adopting the GDR2 parallax of $\delta$ Cep B as that of the system, they correspond to tangential velocity anomalies of $\Delta v_{\text {tan,Hip }}=2.1 \pm 0.4 \mathrm{~km} \mathrm{~s}^{-1}$ and $\Delta v_{\mathrm{tan}, \mathrm{G} 2}=5.3 \pm 1.5 \mathrm{~km} \mathrm{~s}^{-1}$. These velocities are too large to be induced by the orbital motion of the distant companion $\delta$ Cep B. In Paper I, the mass of the close-in companion $\delta$ Cep Ab was estimated to $m_{A b}=0.72 \pm 0.11 M_{\odot}$, assuming a mass of $m_{A}=4.80 \pm 0.72 M_{\odot}$ for the CC. This corresponds to a red dwarf between the spectral types K3V and M0V (Pecaut \& Mamajek 2013). This late spectral type explains why this companion could not be detected by Gallenne et al. (2016) using optical interferometry.

Based on the observed PMa, we confirm that $\delta$ Cep is probably a quadruple system, pending the confirmation that component $\mathrm{B}$ is gravitationally bound to $\mathrm{A}$.

\subsubsection{CP Cep}

Madore (1977) argued that the long-period pulsator CP Cep is a binary with a B3 spectral type companion. From Paper I, CP Cep $(P=17.86 \mathrm{~d})$ exhibits a significant PMa with a $\mathrm{S} / \mathrm{N}$ of $\Delta_{\mathrm{Hip}}=3.2$. We therefore confirm the binarity of this $\mathrm{CC}$ with a close-in orbiting companion. We do not detect additional resolved companions in the GDR2 catalog.

\subsubsection{AX Cir}

We reported in Paper I the presence of a strong PMa on AX Cir induced by its close-in orbiting companion (Jaschek \& Jaschek 1960; Petterson et al. 2004) of spectral type B6V (Evans 1994). This companion was resolved using optical interferometry by Gallenne et al. (2014a). The combination of the GDR2 astrometric PM vectors and the spectroscopic orbit enabled us in Paper I to determine that the close-in companion of AX Cir has a mass of $\approx 5.2 M_{\odot}$, larger than that of the Cepheid. This value, compared with the mass from a STIS spectrum (in prep.) of $3.5 M_{\odot}(\mathrm{B} 9 \mathrm{~V})$, suggests that the companion is itself a binary.

We also identified a very faint Bound candidate (Fig. A.1) at a large projected separation of $42 \mathrm{kau}$, whose spectral type is M3.5V (Table A.1).

\subsubsection{BP Cir}

The short-period BP Cir $(P=2.398 \mathrm{~d})$ is a known binary star through its changing $\gamma$-velocity (Petterson et al. 2004), although its orbital elements are still uncertain. In Paper I, we did not detect any significant PMa, possibly due to the very long orbital period. Gallenne et al. (2018b) detected a close-in companion of BP Cir from near-infrared interferometry, at a separation corresponding to an orbital period of $\approx 14680 \mathrm{~d}$.

We identified a very low mass common proper motion candidate companion of spectral type $\mathrm{M} 2 \mathrm{~V}$ at a large projected separation of $39 \mathrm{kau}$, with a parallax of $\left.\varpi_{\mathrm{G} 2}=1.53 \pm 0.19 \mathrm{mas}\right)$. We note that the GDR2 parallax of BP Cir $\left(\varpi_{\mathrm{G} 2}=1.02 \pm 0.04\right.$ mas $)$ is significantly different from the value derived from the Leavitt law $\left(\varpi_{\mathrm{PL}}=1.70 \mathrm{mas}\right)$.

\subsubsection{R Cru}

$\mathrm{R} \mathrm{Cru}$ is a suspected binary from its $\gamma$-velocity drift (Lloyd Evans 1982). We detected a tight, Bound comoving companion of R Cru (Fig. A.2) of spectral type G8V at a projected 
P. Kervella et al.: Multiplicity of Galactic Cepheids and RR Lyrae stars from Gaia DR2. II.

separation of 7.70" (6.6 kau) from the CC (Table A.1). This companion was also identified by Evans et al. (2016a) from HST imaging at a separation of 7.64". The slight change in separation between their observing epoch (2014.0) and the GDR2 epoch (2015.5) is indicative of a possible orbital motion. The spectral type that we determine for R Cru B is compatible with this star being the source of the X-ray emission detected by Evans et al. (2016b) using XMM imaging.

In addition, we found a marginal $\mathrm{PMa}\left(\Delta_{\mathrm{G} 2}=2.2\right.$; visible in Fig. A.2) that indicates the possible presence of another close-in companion.

\subsubsection{Cru}

The resolved companion of X Cru (Fig. A.2) is a main sequence solar-type dwarf $\left(T_{\text {eff }}=6000 \pm 200 \mathrm{~K}, R=1.0 \pm 0.1 R_{\odot}\right)$ of likely spectral type G1V (Table A.1). It is located at a large projected separation of $40 \mathrm{kau}$ from the CC.

\subsubsection{SU Cru}

SU Cru is a known spectroscopic binary (Szabados 1996) that shows a high PMa $\left(\Delta_{\mathrm{G} 2}=7.4\right.$; Paper I). However, this signal is possibly biased by a significant error in the HIPPARcos PM or position values, as the PM vector in the GDR2 catalog is much lower. We did not detect a resolved companion.

\subsubsection{VW Cru}

VW Cru $(P=5.265 \mathrm{~d})$ is not a known binary CC (Szabados 2003). Its resolved candidate Bound companion is located at a projected separation of $29 \mathrm{kau}$ (Fig. A.2). It is a hot dwarf of spectral type A2V (Table A.1).

\subsubsection{SU Cyg}

SU Cyg is a $3.845 \mathrm{~d}$ period CC that exhibits a very strong PMa of $\Delta_{\mathrm{G} 2}=15.9$. It is a member of the open cluster Turner 9 (Anderson et al. 2013), and a known binary, whose orbital parameters were determined by Evans (1988). The close-in main sequence companion is a $\mathrm{HgMn}$ star (Wahlgren \& Evans 1998) for which we derived a provisional mass of $m_{2}=4.7 \pm 0.7 M_{\odot}$ in Paper I.

Turner et al. (1997) suggested that the close A2V nearby field star (\#1 in their Table 1) may be physically associated with the CC. This source (Gaia DR2 2031776202584173952) is located at a separation of $24.8^{\prime \prime}$, west of the CC (Fig. A.6). Its GDR2 parallax $\left(\varpi_{\mathrm{G} 2}=1.157 \pm 0.034\right.$ mas $)$ is within $1 \sigma$ of the GDR2 parallax of SU Cyg $\left(\varpi_{\mathrm{G} 2}=1.198 \pm 0.052\right.$ mas $)$. The difference in GDR2 tangential velocity between the two stars reaches $d v_{\tan }=4.5 \pm 0.9 \mathrm{~km} \mathrm{~s}^{-1}$, which indicates that this field star is not gravitationally bound to the $\mathrm{CC}$ (the escape velocity is $v_{\mathrm{esc}} \approx 1 \mathrm{~km} \mathrm{~s}^{-1}$ at the projected separation). The difference is even larger if we consider the difference between the proper motion $\mu_{\mathrm{HG}}$ of the CC (mean PM between the HIPPARcos and GDR2 epochs) and the GDR2 PM vector of the A2V field star. However, as argued by Turner et al. (1997), the GDR2 parallax confirms its small physical separation from the CC, which makes it a good fiducial to estimate the color excess $E(B-V)$ of the CC.

\subsubsection{V0532 Cyg}

The short-period Cepheid V0532 Cyg is a suspected binary star (Gorynya et al. 1996a) with a possible period of around $400 \mathrm{~d}$.
We did not detect any significant PMa, but a common proper motion companion of spectral type F0V (Table A.1) is present at a projected separation of $29 \mathrm{kau}$ (Fig. A.2).

\subsubsection{V1046 Cyg}

We identified a close resolved common proper motion companion of V1046 Cyg at a projected separation of $6.7 \mathrm{kau}$ (Fig. A.2). It is a hot dwarf of spectral type $\mathrm{B} 8 \mathrm{~V}$, with a probable mass around 3.5 $M_{\odot}$ (Table A.1).

\subsubsection{V1334 Cyg}

This short-period, first overtone $\mathrm{CC}$ is a known spectroscopic (Evans 1995, 2000) and interferometric (Gallenne et al. 2013, 2018a) binary star, that exhibits the strongest PMa of our sample $\left(\Delta_{\mathrm{G} 2}=31.0\right)$. A detailed discussion of the PMa of V1334 Cyg, and its use to determine the physical parameters of the system, is presented in Paper I.

A common proper motion companion (Gaia DR2 $1964855939153629312)$ is present at a separation of $66^{\prime \prime}$ (44 kau, Fig. A.7). Its parallax of $\varpi_{\mathrm{G} 2}=1.409 \pm 0.318$ mas is in good agreement with the value $\varpi_{\mathrm{G} 18}=1.388 \pm 0.015 \mathrm{mas}$ determined by Gallenne et al. (2018a). Adopting the same color excess $E(B-V)=0.025 \pm 0.009$ as the CC (Kovtyukh et al. 2008 ), the $G$ band magnitude of the candidate companion $\left(m_{G}=19.7\right)$ is $M_{G}=10.4$. This star is thus likely a low mass red dwarf of early $M$ spectral type. This hypothesis is compatible with its relatively large $G-G_{\mathrm{RP}}=1.1$ color index.

\subsubsection{1. $\zeta$ Gem}

The association of the nearby visual companion HD 268518 (WDS J07041+2034 B) with the $10 \mathrm{~d}$ period CC $\zeta \mathrm{Gem}$ (HD 52973) is disproved by its very different GDR2 parallax $\left(\varpi_{\mathrm{G} 2}=28.65 \pm 0.06 \mathrm{mas}\right)$ and proper motion. It was found to be a spectroscopic binary with an F4V spectral type, and proposed to be a physical companion of the CC by Majaess et al. (2012d). We did not detect any other resolved candidate companions to $\zeta$ Gem. However, $\zeta$ Gem displays a marginal PMa at a level of $\Delta_{\mathrm{G} 2}=2.3$ (Paper I), which indicates the possible presence of a close-in orbiting companion, although it shows no sign of orbital motion (Evans et al. 2015).

\subsubsection{T Mon}

$\mathrm{T}$ Mon is a rare long-period $\mathrm{CC}(P=27.02 \mathrm{~d})$. It exhibits a moderate PMa $\left(\Delta_{\mathrm{G} 2}=2.6\right)$, from which we derived a high companion mass of $8.4 M_{\odot}$ (Paper I), comparable to the mass of the CC. This is in line with the conclusions by Evans et al. (1999) who proposed that component B is a chemically peculiar star of the magnetic Ap $\alpha \mathrm{CVn}$ type, and likely to be a binary itself, as chemically peculiar stars often are. However, no detection was reported from near-infrared interferometry by Gallenne et al. (2018b). We did not identify resolved common proper motion candidates.

\subsubsection{Mon}

$\mathrm{CV}$ Mon is a member of the open cluster van den Bergh 1 (Chen et al. 2015), with a color excess $E(B-V)=$ $0.75 \pm 0.02 \mathrm{mag}$ (Turner et al. 1998). This value is consistent with that of Groenewegen (2013), $E(B-V)=0.722$, which we 
adopt here (Table A.1). It shows a PMa at a level of $\Delta_{\text {Hip }}=2.7$, but of only $\Delta_{\mathrm{G} 2}=2.0$ (Paper I).

As shown in Fig. A.3, we identified two nearby stars, Gaia DR2 3127142224816361600 and Gaia DR2 3127142327895572352 , that are potentially gravitationally bound to the CC. The position angle of the PM vector of the latter differs by $15.7^{\circ}$ from that of the CC. This is slightly more than our acceptable limit for Bound companions $\left(15^{\circ}\right.$ at this separation); nevertheless, we have included it in our Bound list in Table A.1. These two stars were already identified by Evans \& Udalski (1994) as probable companions; they were measured at slightly larger angular separations from the $\mathrm{CC}$, which may be due to their orbital motion. Their tangential velocities relative to the target are below $1 \mathrm{~km} \mathrm{~s}^{-1}$, therefore compatible with being gravitationally bound to the $\mathrm{CC}$ at their respective projected linear separations of 18 and $24 \mathrm{kau}$. The spectral type of both companions is around $\mathrm{B} 8 \mathrm{~V}$, corresponding to a mass of $\approx 3.5 M_{\odot}$ (Pecaut \& Mamajek 2013).

\subsubsection{S Mus}

S Mus $(P=9.66 \mathrm{~d})$ is a known spectroscopic binary (Evans 1990; Bohm-Vitense et al. 1990), and a member of the open cluster ASCC 69 (Anderson et al. 2013; Evans et al. 2014; Chen et al. 2017). We detected a moderate PMa (Paper I), which we used in combination with the spectroscopic orbit of Gallenne et al. (2018b) to determine a mass of $m_{2}=$ $2.2 \pm 0.3 M_{\odot}$ for the secondary star. This is significantly lower than expected from the $\mathrm{B} 3.5 \mathrm{~V}$ spectral type estimated by Evans et al. (1994) from IUE observations, but it should be noted that the short orbital period of S Mus $\left(P_{\text {orb }}=505 \mathrm{~d}\right)$ results in a strong smearing of the PMa, particularly for the HipPARcos epoch. The low mass we determined could therefore be significantly biased and should be considered preliminary. Gallenne et al. (2018b) detected the companion of S Mus from near-infrared interferometry, and established the orbital elements of the system. Epoch astrometry from future Gaia data releases will provide a very accurate astrometric orbit of the photocenter of the system.

The resolved candidate companion reported by Evans et al. (2016b, and in prep.) is not present in the GDR2, possibly due to its angular proximity to the bright $\mathrm{CC}$. Evans et al. concluded that it is not a young companion, but that X-rays are probably produced by the spectroscopic binary companion. We did not detect other Bound candidate companions.

\subsubsection{S Nor}

$\mathrm{S}$ Nor $(P=9.75 \mathrm{~d})$ is known to host a resolved companion at a separation of $0.90^{\prime \prime}$ (equivalent to $\approx 800 \mathrm{au}$ ) discovered by Evans et al. (2013). We did not detect this companion in the GDR2 catalog as it is located too close to the CC. We did not find other, more distant Bound candidate companions, and we do not confirm the companion proposed by Evans \& Udalski (1994). However, S Nor is a member of the open cluster NGC 6087 (Anderson et al. 2013), and is therefore located in a rich stellar field where many Near candidates are present with comparable PM vectors (Fig. A.7).

We observed a significant PMa in the HipParcos $\left(\Delta_{\text {Hip }}=3.8\right)$ and moderate in the GDR2 $\left(\Delta_{\mathrm{G} 2}=2.2\right)$ that confirm the presence of a close-in orbiting component. Combining them with the spectroscopic orbital parameters by Groenewegen (2008) (who assumed a circular orbit), we derived a companion mass of $1.5 \pm 0.2 M_{\odot}$ (Paper I). The PMa is an important confirmation of a close orbit, which has been difficult to identify. There are three well-covered seasons of CORAVEL data from Mermilliod et al. (1987) and Bersier et al. (1994) showing little evidence of orbital motion. No signature of a close-in companion was found by Gallenne et al. (2018b) in radial velocity at a level of $\pm 1 \mathrm{~km} \mathrm{~s}^{-1}$.

\subsubsection{RS Nor}

RS Nor $(P=6.20 \mathrm{~d})$ does not show a significant PMa (Paper I) and is not a known binary. We detected a relatively massive Bound candidate companion of spectral type A1V (Table A.1) at a projected separation of $15 \mathrm{kau}$ (Fig. A.3).

\subsubsection{SY Nor}

SY Nor $(P=12.64 \mathrm{~d})$ is a known binary system (Szabados 2003) with a close-in B4.5V companion identified from IUE observations by Evans (1994). We detected a strong PMa induced by this companion $\left(\Delta_{\mathrm{G} 2}=8.0\right)$.

We also detected two resolved, gravitationally bound candidates (Fig. A.3). The resolved companions have respective projected separations of $5.7 \mathrm{kau}$ and $42 \mathrm{kau}$. The closer resolved candidate (separation $5.7 \mathrm{kau}$ ), Gaia DR2 5884729035245399424 (SY Nor B), was identified by Proust et al. (1981) and is discussed in Appendix A of Evans (1994) as potentially bound to the CC. Its B2V spectral type corresponds to a mass of $\approx 7 M_{\odot}$, which is comparable to the expected $\mathrm{CC}$ mass $\left(\approx 6.2 M_{\odot}\right)$. This high mass may indicate that $\mathrm{B}$ is itself a binary. The $\mathrm{AB}$ pair is present in the WDS catalog (WDS J15548-5434; Mason et al. 2001), and we list in Table 4 the relative position of component B. We confirm that SY Nor $\mathrm{B}$ is gravitationally bound to the $\mathrm{CC}$, and although its orbital period is likely on the order of $100 \mathrm{ka}$, its astrometric orbital displacement may be detectable in the future Gaia data releases or, for example, by using the astrometric dual field mode of the GRAVITY instrument (Gravity Collaboration 2017).

The wider candidate companion (42 kau), of approximate spectral type F6V, marginally satisfies the gravitational bound condition on the tangential velocity. However, considering that the inner system is already composed of at least three massive stars with a total mass on the order of $20 M_{\odot}$, we classify this star as a Bound candidate. SY Nor is therefore likely a hierarchical quadruple system, possibly quintuple if B is a binary.

\subsubsection{QZ Nor}

The short-period, first overtone (Klagyivik \& Szabados 2009) Cepheid QZ Nor $(P=3.786 \mathrm{~d})$ is not known to be a binary system (Szabados 2003). It probably belongs to the open cluster NGC 6067 (Eggen 1983; Anderson et al. 2013), together with the Cepheid V0340 Nor. We identified a low mass (spectral type K1V, Table A.1), common proper motion companion at a large projected separation of $30 \mathrm{kau}$ (Fig. A.3). Its GDR2 parallax is compatible with that of the CC within their uncertainties.

\subsubsection{Y Oph}

Y Oph is a long-period CC $(P=17.12 \mathrm{~d})$ with peculiar sinusoidal photometric and radial velocity curves. It shows phase jumps in its $O-C$ diagram (Szabados 1989), and exhibits a strong reddening, that may be partly of circumstellar origin. Abt \& Levy (1978) and Pel (1978) proposed that Y Oph 
P. Kervella et al.: Multiplicity of Galactic Cepheids and RR Lyrae stars from Gaia DR2. II.

Table 4. Relative positions of SY Nor B with respect to SY Nor A.

\begin{tabular}{lll}
\hline \hline Epoch & PA $\left({ }^{\circ}\right)$ & Separation $\left({ }^{\prime \prime}\right)$ \\
\hline 1934 & 315 & 2.1 \\
1967 & 315 & 2.2 \\
2015.5 & $314.7862 \pm 0.0015$ & $2.46341 \pm 0.00007$ \\
\hline
\end{tabular}

is a spectroscopic binary star, which was not confirmed by Evans \& Lyons (1986). IUE observations by Evans (1992b) excluded the possibility of a close-in companion hotter than spectral type A0, but Szabados (1989) determined a possible binary orbital period of $1223 \mathrm{~d}$. We did not detect any significant PMa in Y Oph $\left(\Delta_{\mathrm{G} 2}=0.7\right.$; Paper I), and we thus do not confirm its binarity.

The GDR2 parallax of Y Oph $\left(\varpi_{\mathrm{G} 2}=1.39 \pm 0.08\right.$ mas $)$ is different from the measurements obtained using the classical Baade-Wesselink technique (e.g., $\varpi=1.79 \pm 0.09$ mas from Groenewegen 2013, $\varpi=1.81 \pm 0.13$ mas from Fouqué et al. $2007)$ or its interferometric version $(\varpi=2.04 \pm 0.07 \mathrm{mas}$; Mérand et al. 2007). Considering this uncertainty on the GDR2 parallax, we considered a broad parallax range from $\varpi=1.2$ to 2.5 mas as prior for the wide companion search. No Bound companion was detected assuming this prior condition on the parallax. The only field star showing a proper motion comparable to that of Y Oph is Gaia DR2 4175017934690530688, a faint red dwarf. With a parallax of $\varpi_{\mathrm{G} 2}=1.726 \pm 0.395$ mas, it is compatible with our adopted range of acceptable parallaxes. Future Gaia data releases will provide more accurate parallax values of both this star and Y Oph and confirm if they are comoving companions. We note that Gallenne et al. (2014b) did not find any companion of Y Oph from adaptive optics imaging up to a projected separation of $1.7^{\prime \prime}$.

\subsubsection{AW Per}

AW Per is a fundamental mode $\mathrm{CC}$ with a pulsation period of $P_{\text {puls }}=6.46 \mathrm{~d}$. It is a known spectroscopic binary system (Welch \& Evans 1989). Evans et al. (2000) determined accurate orbital parameters of the inner AW Per Aab system and in particular an orbital period of $P_{\text {orb }} \approx 40 \mathrm{yr}$. They determined a minimum dynamical mass of $6.6 M_{\odot}$ for the CC's spectroscopic companion $\mathrm{Ab}$, which is significantly higher than the mass inferred from its spectral type $\left(4.0 M_{\odot}\right)$. They therefore proposed that the Ab component is itself a binary system. Massa \& Evans (2008) measured the position angle and separation of the companion from the $\mathrm{CC}$ at epoch November 2001 (MJD 52235): $\theta=13.74 \pm 0.26$ mas, $\mathrm{PA}=184 \pm 2^{\circ}$. Gallenne et al. (2015) detected the companion of AW Per using near-infrared interferometry. We detected a very strong PMa $\left(\Delta_{\mathrm{G} 2}=21.4\right.$; Paper I), that we attribute to the photocenter displacement of the inner Aab system. The combination of the spectroscopic orbital parameters from Griffin (2016) with the PM anomalies (Paper I) confirm the high mass of the companion $\left(8.8 \pm 1.3 M_{\odot}\right)$, and consequently its binarity.

We detected an additional high probability Bound candidate companion at a projected linear separation of 8.4 kau (Fig. A.3). This is a low mass star whose spectral type is likely around K3.5V. AW Per is therefore likely a hierarchical quadruple system.

\subsubsection{RS Pup}

The long-period Cepheid RS Pup $(P \approx 41.5 \mathrm{~d})$ is one of the most luminous Galactic CCs. It is remarkable as it is embedded in a large dusty nebula that scatters the light from the pulsating star creating light echoes. This phenomenon was discovered by Westerlund (1961) and first studied by Havlen (1972). Kervella et al. (2014) determined the distance to the $\mathrm{CC}\left(d_{\text {echoes }}=1910 \pm 80 \mathrm{pc}\right)$ using polarimetric imaging of the light echoes with the HST ACS camera. The presence of light echoes implies that the nebula is associated with the $\mathrm{CC}$, but it is too massive to have been created by mass loss from the star itself (Kervella et al. 2012). The extension of the nebula $(\approx 1 \mathrm{pc})$ is negligible with respect to the distance to the CC $(\approx 2 \mathrm{kpc})$. Kervella et al. (2017b) determined the spectroscopic projection factor of RS Pup $p=1.25 \pm 0.06$ and its color excess $E(B-V)=0.496 \pm 0.006$.

The star Gaia DR2 5546476755539995008 (hereafter S1) appears to be embedded within the nebula surrounding RS Pup because it is interacting with the dust cloud (Fig. 4). The differential proper motion with respect to RSPup is $\mu[\mathrm{S} 1-\mathrm{RS}$ Pup $]=(-0.15,+3.26) \mathrm{mas} \mathrm{a}^{-1}$. This corresponds to a tangential velocity difference of $\approx 30 \mathrm{~km} \mathrm{~s}^{-1}$, and $\mathrm{S} 1$ is therefore not gravitationally bound to the CC. Its GDR2 parallax is $\varpi[\mathrm{S} 1]=0.532 \pm 0.048$ mas, and its spectral type is F6V (Table A.1). The presence of this star in the nebula makes it an interesting proxy for the GDR2 parallax of RS Pup, without the possible bias due to the large amplitude photometric and color variability of the CC. We note that the parallax of S1 is compatible within the error bars with that of the $\mathrm{CC}$ determined from polarimetric imaging of the light echoes $\varpi_{\text {echoes }}=$ $0.524 \pm 0.022$ mas (Kervella et al. 2014).

RS Pup shows a moderate PMa $\Delta_{\mathrm{G} 2}=1.8$ (Paper I), which indicates the possible presence of a close-in orbiting companion. However, the presence of the bright light echoes close to the CC may perturb the Gaia astrometric measurements.

\subsubsection{S Sge}

We detected a very strong PMa of $\Delta_{\mathrm{G} 2}=20.0$ (Paper I) on S Sge $(P=8.38 \mathrm{~d})$. Combined with the spectroscopic orbital parameters from Groenewegen (2008), we derived an inclination of the orbital plane of $i=75 \pm 10 \mathrm{deg}$, and a companion mass of $3.0 \pm 0.5 M_{\odot}$. This mass is still higher than that from the IUE spectrum (1.7-1.5 $M_{\odot}$; Evans et al. 1993), making it likely that the companion is itself a binary; however, the short orbital period of $676 \mathrm{~d}$ results in a temporal smearing of the HiPPARcos PMa vector, and this mass should be considered provisional. We did not detect any resolved Bound candidate companions.

\subsubsection{U Sgr}

U Sgr $(P=6.75 \mathrm{~d})$ does not display a significant PMa. It is a member of the open cluster Messier 25 (IC 4725; Irwin 1955; Anderson et al. 2013). We identified a number of field comoving stars of the cluster that are visible in Fig. A.3, two of which are Bound candidates. These are early A-type dwarf or subgiant stars (Table A.1).

\subsubsection{V0350 Sgr}

We detected a very strong PMa in V0350 Sgr $\left(\Delta_{\mathrm{G} 2}=8.7\right.$; Paper I) induced by its known close-in companion (Evans et al. 2015, 2018c). The combination of the astrometric PM anomalies and the spectroscopic orbit by Gallenne et al. (2018b) results in a mass of $m_{2}=3.8 \pm 0.6 M_{\odot}$ for the secondary. This mass is compatible with the $\mathrm{B} 9.5 \mathrm{~V}$ spectral type determined by 


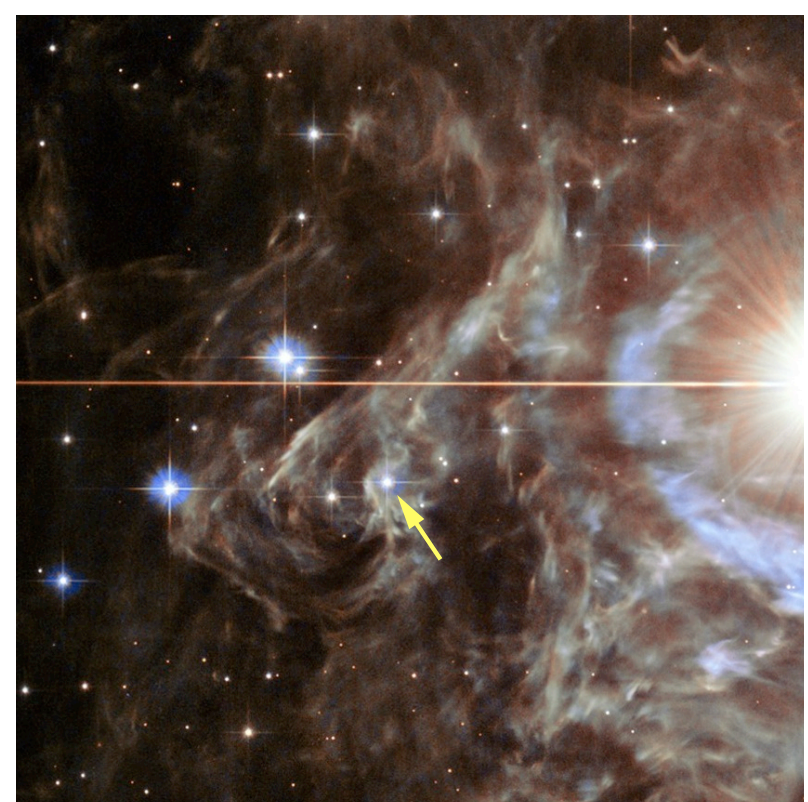

Fig. 4. Star S1 (yellow arrow) associated with the dusty nebula surrounding the long-period Cepheid RS Pup (the bright star at the right edge of the image). Color rendition: NASA, ESA, Z. Levay, and the Hubble Heritage Team STScI/AURA-Hubble/Europe Collaboration.

Evans et al. (2013) from IUE observations, within the error bars. A possible interferometric detection of the companion was reported by (Gallenne et al. 2018b). We also found a Bound candidate at a projected separation of $26 \mathrm{kau}$ (Fig. A.4) that has a spectral type of A2V (Table A.1). V350 Sgr is therefore probably a triple system.

\subsubsection{W Sgr}

W Sgr is a $P=7.6 \mathrm{~d}$ CC that is a member of a triple system, with one wide (A0V spectral type; Morgan et al. 1978; Bohm-Vitense \& Proffitt 1985; Evans 1991) and one spectroscopic companion (discovered by Babel et al. 1989). Gallenne et al. (2018b) determined the spectroscopic orbital parameters of the inner system. Combined with the moderate PMa of the star, the spectroscopic companion mass was estimated to $m_{2}=1.1 \pm 0.2 M_{\odot}$ (Paper I). The known wide component of W Sgr is too close to the CC for Gaia $\left(<0.2^{\prime \prime}\right)$, and we did not detect other Bound candidate companions.

\subsubsection{RV Sco}

The very strong PMa that we detected $\left(\Delta_{\mathrm{G} 2}=9.7\right.$; Paper I) confirms that this star is a close binary. Szabados (1989) proposed an orbital period of $\approx 8000 \mathrm{~d}$ from the residuals of the $O-C$ diagram. Evans (1992b) established an upper limit of A3 for possible main sequence companions of RV Sco. We note that the HIPPARcos and GDR2 PM anomalies are significantly different (time separation of $8850 \mathrm{~d}$ ), and the future Gaia data releases will provide the necessary astrometry to improve the determination of the companion properties. We did not identify any resolved Bound candidates from the GDR2 catalog.

\subsubsection{V0636 Sco}

From its strong PMa $\left(\Delta_{\mathrm{G} 2}=11.9\right.$; Paper I $)$ and the spectroscopic orbital parameters from Gallenne et al. (2018b), we determined that the close-in companion of this $P=6.80 \mathrm{~d}$ pulsator has a mass of $m_{2}=2.3 \pm 0.3 M_{\odot}$. This estimate is in good agreement with the determination by Böhm-Vitense et al. (1998) who found a spectral type of $\mathrm{B} 9.5 \mathrm{~V}\left(m_{2} \approx 2.5 M_{\odot}\right)$ for V0636 Sco B using UV spectroscopy from the HST. A possible interferometric detection of this companion is reported by Gallenne et al. (2018b). We did not identify any resolved Bound candidate from the GDR2 catalog.

\subsubsection{V0950 Sco}

We did not find a PMa $\left(\Delta_{\mathrm{G} 2}=1.8\right.$; Paper I) on V0950 Sco. We isolated one resolved Bound candidate, Gaia DR2 5960623340819000192 (Fig. A.4) located at a projected separation of $15 \mathrm{kau}$ from the CC and of spectral type G1V (Table A.1).

\subsubsection{Sct}

CM Sct is a short-period CC $(P=3.92 \mathrm{~d})$ that is not classified as a known spectroscopic binary, in line with our non detection of any significant PMa (Paper I).

We detected a resolved Bound candidate companion of spectral type A1V (Table A.1), Gaia DR2 4253603428053877504 (Fig. A.4), located at a projected separation of $49 \mathrm{kau}\left(26.8^{\prime \prime}\right)$. The GDR2 parallax of this companion $(\varpi=0.547 \pm 0.050$ mas) is slightly larger than that of the CC $(\varpi=0.405 \pm 0.065$ mas $)$. Considering that the expected parallax from the Leavitt law is $\varpi=0.518$ mas, this difference may be due to an offset in the GDR2 parallax of the CC.

\subsubsection{EV Sct}

EV Sct is a member of the open cluster NGC 6664 (Turner 1976; Mermilliod et al. 1987; Hoyle et al. 2003; Anderson et al. 2013), and the GDR2 parallax value $(\varpi=0.526 \pm 0.054$ mas $)$ confirms this association. As a consequence, the stellar density is high in the field around EV Sct, and we detected a number of LowV sources with a similar proper motion as the CC (Fig. A.4). We identified one candidate Bound companion of spectral type B9V (Table A.1), Gaia DR2 4156513016572003840, that is located at a projected separation of $44 \mathrm{kau}\left(22.6^{\prime \prime}\right)$ from the primary.

Kovtyukh \& Andrievsky (1999) proposed that EV Sct is a binary comprising two $\mathrm{CCs}$, the companion being itself a very short-period CC $(P=1.2 \mathrm{~d})$. We detected only a $\Delta_{\mathrm{G} 2}=1.1$ PMa on EV Sct, and we therefore cannot confirm the presence of a close-in orbiting companion. Future Gaia data releases will provide a stringent test of the presence of this companion.

\subsubsection{SZ Tau}

SZ Tau is a suspected spectroscopic binary star of low radial velocity amplitude (Gorynya et al. 1996a,b). It is also a member of the open cluster NGC 1647 (Efremov 1964; Turner 1992; Anderson et al. 2013). Evans (1992b) placed an upper limit of A1 on the spectral type of a main sequence companion, and Evans et al. (2016b) found little evidence of orbital motion (to about $\left.\pm 1 \mathrm{~km} \mathrm{~s}^{-1}\right)$. The moderate PMa that we observed $\left(\Delta_{\mathrm{G} 2}=\right.$ 1.9; Paper I) is not conclusive.

\subsubsection{Polaris ( $\alpha$ UMi)}

Polaris Aa ( $\alpha$ UMi, HD 8890, HIP 11767) is the nearest CC, but with a surprising uncertainty in its distance. Its parallax 
has been measured by Hipparcos, and was found to be $\varpi_{\text {Hip }}=$ $7.54 \pm 0.11$ mas (van Leeuwen 2007a,b). Unfortunately, Polaris A is too bright for Gaia, but the parallax of its physical companion Polaris $\mathrm{B}$ has been measured at $\varpi_{\mathrm{G} 2}=7.321 \pm 0.028$ mas (value corrected for the $29 \mu$ as ZP offset). This value is larger by $4.4 \sigma$ than the parallax $\varpi_{\mathrm{FGS}}=6.26 \pm 0.24$ mas for the same star measured by Bond et al. (2018) using the HST FGS. With the agreement between the HIPPARcos and Gaia results, the FGS parallax is an outlier, but the reasons for this are unclear. A discussion of the parallaxes of Polaris A and B is presented in Appendix B of Groenewegen (2018) and in Engle et al. (2018). Polaris B is a fast rotating main sequence F3V star (Usenko \& Klochkova 2008). Polaris is part of a tight binary system with the secondary component Polaris Ab (Evans et al. 2002, 2008, 2018a). Using the Gaia distance, Evans et al. (2018a) found a preliminary mass of $3.45 \pm 0.75 M_{\odot}$. Using the FGS parallax, the likely mass is $6.5-7 M_{\odot}$, and the Cepheid is pulsating in the first overtone and crossing the instability strip for the first time (Anderson 2018; Engle et al. 2018).

For the present companion search, we adopt the HIPPARcos parallax $\varpi_{\text {Hip }}=7.54$ mas as our prior estimate, with a $15 \%$ relative uncertainty $( \pm 1.1$ mas $)$. This parallax range is compatible with the GDR2 parallax of Polaris B, whose properties are listed in Table A.1. This choice of prior parallax has a low influence on the efficiency of the search algorithm. This is particularly true as Polaris is located far from the plane of the Milky Way, and therefore in a low star density field. We also adopt the Hipparcos proper motion vector $\mu_{\text {Hip }}=[+44.48 \pm 0.11,-11.85 \pm 0.13] \mathrm{mas} \mathrm{a}^{-1}$ (van Leeuwen 2007a). The field around Polaris is presented in Fig. 5. The differential tangential velocity of Polaris B with respect to $\mathrm{A}$ is $d v_{\tan }=1.96 \pm 0.32 \mathrm{~km} \mathrm{~s}^{-1}$. The radial velocities determined by Kamper (1996) of Polaris A ( $\gamma$-velocity of the inner binary system $\left.v_{\text {rad }}=-16.42 \pm 0.03 \mathrm{~km} \mathrm{~s}^{-1}\right)$ and Polaris B $\left(v_{\text {rad }}=-14.7 \pm 1.2 \mathrm{~km} \mathrm{~s}^{-1}\right)$ correspond to a differential radial velocity $d v_{\text {rad }}=1.7 \pm 1.2 \mathrm{~km} \mathrm{~s}^{-1}$. This results in a space velocity of Polaris B with respect to A of $d v=2.6 \pm 1.2 \mathrm{~km} \mathrm{~s}^{-1}$. The escape velocity at the projected separation of $2.3 \mathrm{kau}$ is $v_{\mathrm{esc}} \approx 3.2 \mathrm{~km} \mathrm{~s}^{-1}$, assuming a total mass of $6.7 M_{\odot}$ for the three components. We thus conclude that the Polaris B is likely gravitationally bound to A, thus forming a triple stellar system.

\subsubsection{SX Vel}

SX Vel $(P=9.55 \mathrm{~d})$ is not classifed as a known binary, but it shows a PMa at a level of $\Delta_{\text {Hip }}=2.9$. It is however not present in the GDR2 $\left(\Delta_{\mathrm{G} 2}=1.0\right)$. It was tested for being a member of the open cluster SAI 94 by Anderson et al. (2013), but the association has not been confirmed. We detected a resolved Bound candidate companion of SX Vel at a projected separation of $32 \mathrm{kau}$, which is probably a solar-like star of spectral type G1V.

\subsubsection{CS Vel}

CS Vel is not classified as a spectroscopic binary Cepheid. It is possibly a member of the open cluster Ruprecht 79 (Turner 2010), but this association is considered inconclusive by Anderson et al. (2013). It is also discussed in Chen et al. (2015), who reach the same conclusion, but based on an incorrect PM vector. The GDR2 $\mathrm{PM}$ vector of CS Vel is $\mu=[-4.56 \pm 0.06,+3.10 \pm 0.06] \mathrm{mas} \mathrm{a}^{-1}$, which is actually consistent within the mean proper motion of the cluster $\mu=[-5.66 \pm 4.67,+4.04 \pm 4.93]$ mas a $^{-1}$. The GDR2 parallax of CS Vel $(\varpi=0.194 \pm 0.030$ mas $)$ is however too

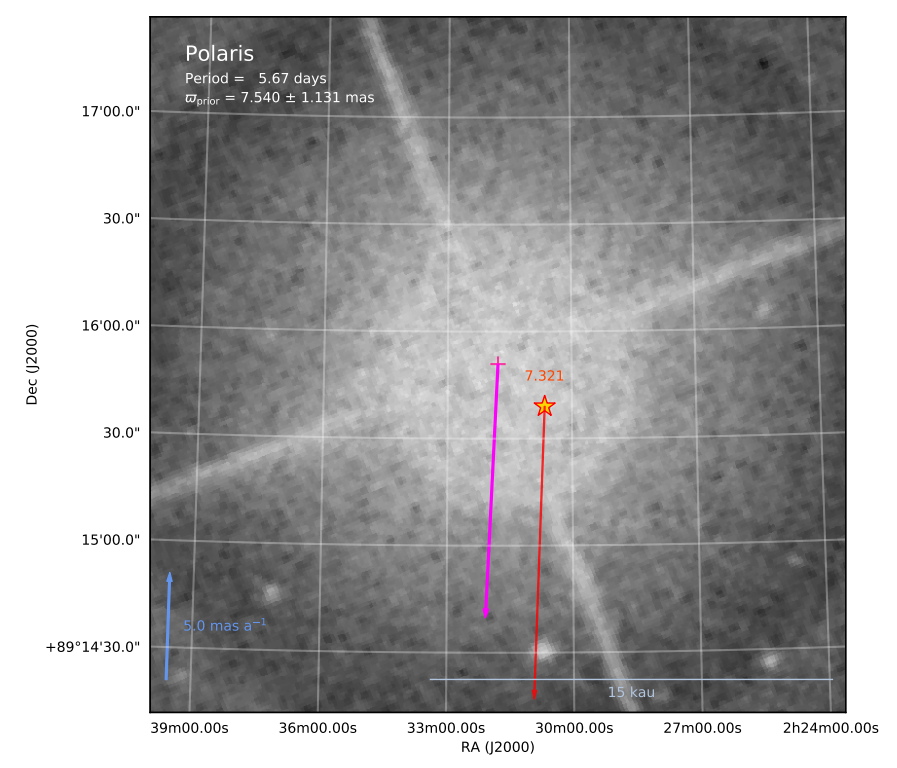

Fig. 5. Field around Polaris from the DSS2-Red showing its companion Polaris B (yellow star).

small compared to the estimated distance of Ruprecht $79(\approx 2 \mathrm{kpc}$; Kharchenko et al. 2016).

We detected a Bound candidate companion of CS Vel (Fig. A.4) located at a projected separation of $36 \mathrm{kau}$ (8.9'), and of spectral type A0V (Table A.1).

\subsubsection{DK Vel}

This short-period CC $(P=2.48 \mathrm{~d})$ is not a known spectroscopic binary, and we detected a marginal PMa $\left(\Delta_{\mathrm{G} 2}=2.1\right.$; Paper I). We identified a low mass Bound candidate companion of DK Vel of spectral type K0V (Table A.1) located at a large projected separation of $50 \mathrm{kau}$ (Fig. A.5).

\subsubsection{U Vul}

Known since the 19th century (Pickering \& Wendell 1899), U Vul is an intermediate period CC $(P=7.99 \mathrm{~d})$. It is classified as a spectroscopic binary (Szabados 1991; Imbert 1996). From its strong PMa and the spectroscopic orbital parameters by Groenewegen (2008), we determined in Paper I an inclination of the orbital plane of $i=163 \pm 7 \mathrm{deg}$. Our estimate of the mass of the companion of $2.4 \pm 0.4 M_{\odot}$ is consistent with the upper limit of $2.1 M_{\odot}$ by Evans et al. (2015) (see also Evans 1992b). We did not detect a resolved common proper motion companion of $U$ $\mathrm{Vul}$ in the GDR2 catalog.

\subsection{RR Lyrae stars}

\subsubsection{AT And}

AT And is an RRab pulsator $(P=0.6169 \mathrm{~d})$ that shows the strongest PMa of all the tested RRLs $\left(\Delta_{\mathrm{G} 2}=16.7\right.$, Paper I). This PMa corresponds to a maximum orbital period of around 6 years (Table A.7 in Paper I) and a maximum angular semimajor axis on the order of 7 mas. This angular separation is in principle within the capabilities of the CHARA Array (ten Brummelaar et al. 2005). However, this a relatively faint $\operatorname{star}\left(m_{V}=10.60\right.$, $\left.m_{K}=9.08\right)$ for the sensitivity of optical interferometers, and the companion may be a very faint compact object that would be difficult to detect. The most promising way to determine 
the orbital parameters would be to follow the evolution of its $\gamma$-velocity over $\approx 6$ yr using spectroscopy. AT And exhibits the Blazhko effect (Greer et al. 2017), as well as period change (Olah \& Szeidl 1978). Fernley \& Barnes (1997) proposed that this star is an anomalous Cepheid, but this was not confirmed by Peña \& Peniche (2004). We did not detect any additional resolved companion of AT And from the GDR2 catalog.

\subsubsection{OV And}

OV And is an RRab star $(P=0.4706 \mathrm{~d})$ that does not show the Blazhko effect (Skarka 2014a). This star is absent from the HipParcos catalog, so we could not test for the presence of a PMa as described in Paper I. The PM vector of the first Gaia data release $\mu_{\mathrm{G} 1}=\left[-5.32_{0.88},-8.22_{0.47}\right]$ mas a $^{-1}$ (Gaia Collaboration 2016) is however slightly different from the PM vector of the GDR2 $\mu_{\mathrm{G} 2}=\left[-4.868_{0.057},-7.672_{0.023}\right]$ mas a $^{-1}$ (uncorrected for frame rotation). A more sensitive search for the presence of a PMa will be possible using the future Gaia data releases.

The resolved companion of OV And (Fig. B.1) is a relatively bright and hot star of spectral type F4V (Table B.1), with a probable mass around $1.4 M_{\odot}$. The presence of such a massive companion orbiting an old population RRL, at a linear projected separation of $3.7 \mathrm{kau}$, raises interesting questions on the evolutionary scenario that led to the current state of the system.

\subsubsection{V0363 Cas}

V0363 Cas is classified as an RR(B) pulsator in the GCVS, but it is listed as an RRc in Wils et al. (2006), and as a DCEPS(B) in the VSX database (Watson et al. 2006). A light curve of V0363 Cas was obtained by Hajdu et al. (2009) from the Optical Monitoring Camera on board the INTEGRAL satellite ${ }^{4}$. They showed that this star is pulsating in two radial modes with a 0.802 period ratio of the first to second overtone periods: $P_{1}=0.546556 \mathrm{~d}$ and $P_{2}=0.438243 \mathrm{~d}$. This indicates that V0363 Cas is probably a very short-period CC, hence its variable type in the VSX database, rather than an RRL. We detected a strong PMa $\left(\Delta_{\mathrm{G} 2}=4.7\right.$; Paper I) on V0363 Cas, which indicates that it is a close binary system.

\subsubsection{XZ Cyg}

The RRab pulsator XZ Cyg has a period of $P=0.4667 \mathrm{~d}$ and shows the Blazhko effect (Smith 2006). This is a particularly remarkable RRL due to its extremely fast rising from minimum $\left(m_{V}=10.5\right)$ to maximum $\left(m_{V}=8.8\right)$ brightness in only $53 \mathrm{~min}$ (Huziak 2006). Its light curve presented in LaCluyzé et al. (2004) shows that its Blazhko period $(\approx 57.4 \mathrm{~d})$ is changing with time.

We detected a strong PMa $\left(\Delta_{\mathrm{G} 2}=6.8\right.$; Paper I), which reveals the presence of a close-in binary companion. Depending on their orbital separation, the presence of this secondary massive body may play a role in the observed change of the Blazhko period of the RRL.

\subsubsection{CS Del}

CS Del is classified as an RRc pulsator (Kemper 1982) with a period $P=0.366 \mathrm{~d}$; however, the light curve reported by Bramich et al. (2014) is not conclusive. The resolved companion of CS Del that we identified is located at a projected distance of $5 \mathrm{kau}$ (Fig. B.1) and has a spectral type of G7V (Table B.1).

\footnotetext{
4 Light curve available from http://sdc. cab.inta-csic.es/omc/ var/4014000075.html
}

\subsubsection{V0893 Her}

The classification of V0893 Her as an RR: pulsator $(P=$ $0.4918 \mathrm{~d}$; Maintz 2005) appears uncertain, but its absolute magnitude is compatible with this possibility. Its GDR2 parallax of $\varpi=2.68 \pm 0.03$ mas places it among the nearest RRLs. Its resolved companion (Fig. B.1) is a very low mass red dwarf (spectral type M1.5V), located at a projected separation of only $1.9 \mathrm{kau}$ (Table B.1).

\subsubsection{CZ Lac}

$\mathrm{CZ}$ Lac is listed in the GDR2 as a fundamental mode RRL with a period of $P=0.432 \mathrm{~d}$. It was shown by Sódor et al. (2011) to exhibit an original multiperiodic Blazhko effect (see also Gillet 2013). CZ Lac shows a strong PMa of $\Delta_{\mathrm{G} 2}=3.9$, which indicates the probable presence of a close-in orbiting companion. Its K1V resolved Near companion (Fig. B.3), Gaia DR2 2000976545410515584 is located at an angular separation of $19.4^{\prime \prime}$, which corresponds to nearly 25 kau (Table B.1). Its GDR2 parallax $\varpi_{\mathrm{G} 2}=0.773 \pm 0.050$ mas is slightly smaller than that of the RRL $\varpi_{\mathrm{G} 2}=0.852 \pm 0.029$ mas, but the latter may be affected by the detected PMa. Considering its wide separation, the resolved companion is unlikely to be the cause of the observed PMa, and CZ Lac is therefore possibly a triple system.

\subsubsection{RR Leo}

RR Leo is listed in the Skarka (2014b) catalog, which gives a mass estimate of $m=0.55 \pm 0.02 M_{\odot}$. It has been observed by the HST (Freedman 2013: Prog ID: 13472, PI: Freedman), but unfortunately the field of view is too small to include the companion that we identified as a Bound candidate, Gaia DR2 630421931138065280 (Fig. B.1). The proper motion of the two stars is faster than 18 mas a $^{-1}$, which excludes a chance association. The companion is located at a separation of $9.9^{\prime \prime}$ from the RRL (9.3 kau) and is extremely faint in the visible, with a contrast of $\Delta m_{G}=7.1$ (Table B.1). The contrast in the infrared is reduced to $\Delta m_{K}=5.5$, indicating that the companion is a red dwarf of M0V spectral type. RR Leo does not show a significant $\mathrm{PMa}$ and it is therefore likely a system of only two components.

\subsubsection{V0764 Mon}

The PMa that we detected on V0764 Mon is the second largest in our sample, at $\Delta_{\mathrm{G} 2}=7.1$ (Paper I) and $\Delta_{\mathrm{Hip}}=3.8$. It is classified as an RRc pulsator of period $P=0.290 \mathrm{~d}$ (Maintz 2005). Considering the very strong signature on the PMa detected both at HIPPARcos and GDR2 epochs, V0764 Mon is a certain binary star. A spectroscopic monitoring of its $\gamma$-velocity should easily reveal its orbital motion.

\subsubsection{SS Oct}

SS Oct (HIP 108057) is a $P=0.622 \mathrm{~d}$ RRL showing the Blazhko effect with a period $P_{\text {Blazhko }}=144.930 \mathrm{~d}$ (Skarka 2013). It is classified in the GDR2 as a fundamental mode pulsator. SS Oct is also listed in the Skarka (2014b) catalog, which gives a mass estimate of $m=0.58 \pm 0.03 M_{\odot}$. Its close companion (Fig. B.1), Gaia DR2 6345324695303800192, is a red dwarf of spectral type K2V (Table B.1), 5.4 magnitudes fainter than the RRL in the visible $\left(m_{G}=17.19\right)$. Their common proper motion is very fast at more than 32 mas a $^{-1}$, making a chance association impossible, and the projected physical separation between the two stars is $2.4 \mathrm{kau}$. SS Oct also presents a marginal PMa at $\Delta_{\mathrm{G} 2}=2.2$, and 
P. Kervella et al.: Multiplicity of Galactic Cepheids and RR Lyrae stars from Gaia DR2. II.

the future Gaia data releases will confirm whether it has a close companion in addition to the resolved one.

\subsubsection{EY Oph}

EY Oph is a poorly studied variable, classified as an RR: type star. Its absolute magnitude is faint for an RRL, but may be compatible with this classification if, for example, the adopted color excess $E(B-V)$ is biased. The candidate Bound companion of EY Oph, Gaia DR2 6029835295648727168, is located at a projected separation of $6.6 \mathrm{kau}$ and has a remarkably low absolute $G$ band magnitude of $\approx+10$. This absolute magnitude corresponds to a very low mass M3V star on the main sequence, but its blue $G_{\mathrm{BP}}-G_{\mathrm{RP}}$ color indicates that it is located significantly below the main sequence. This star could therefore be a white dwarf, but in absence of infrared magnitudes, its identification is uncertain.

\subsubsection{V0487 Sco}

V0487 Sco is a c-type RRL with a pulsation period of $P=$ $0.329 \mathrm{~d}$ (Maintz 2005) and a metallicity of $[\mathrm{Fe} / \mathrm{H}]=-1.89$ (Morgan et al. 2007). We detected a resolved companion (Gaia DR2 4053551410541112192) located at a wide projected separation of $28 \mathrm{kau}$. It is only $0.6 \mathrm{mag}$ fainter than the RRL in the $K$ band, but its $G$ band magnitude is fainter than the RRL by $4.7 \mathrm{mag}$. It could be a dusty red giant star with a strong circumstellar absorption differing from that of the RRL.

\subsubsection{AR Ser}

AR Ser is a fundamental mode (Szczygieł et al. 2009) RRab type pulsator $(P=0.5752 \mathrm{~d})$ showing the Blazhko effect (Skarka 2013; Bonnardeau \& Hambsch 2015; Skarka et al. 2016); however, it is not listed as a Blazhko star in the SuperWASP catalog (Greer et al. 2017). We detected a strong PMa of $\Delta_{\mathrm{G} 2}=5.2$, indicative of the presence of an orbiting companion. The modulation period $P_{m}=1325 \pm 60 \mathrm{~d}$ proposed by Skarka et al. (2016) may correspond to the orbital period of the companion. We did not detect a resolved common proper motion candidate in the GDR2 catalog.

\subsubsection{TU UMa}

TU UMa is the only RRL member of a binary system whose spectroscopic orbit is available (Szeidl et al. 1986; Kiss et al. 1995; Wade et al. 1999; Liška et al. 2016a). The results of the combined fit of its PMa and spectroscopic orbit are presented in Table 3 and Sect. 3.5 of Paper I. We determined that its companion is likely a massive white dwarf $\left(m_{2} \approx 2 M_{\odot}\right)$, orbiting with a semimajor axis of $\approx 11 \mathrm{au}$. We did not detect any additional resolved companion of TU UMa.

\subsection{Other variables}

We briefly discuss here the results of our companion search on a selection of stars classified as CCs or RRLs, but that actually do not belong to these classes.

\subsection{1. $\mathrm{HM} \mathrm{Aql}$}

HM Aql is a poorly studied variable classified as an RRL (Kurochkin 1958; Wils et al. 2006; Gavrilchenko et al. 2014), but whose period is unknown. Its absolute magnitude $M_{G}=$ +2.62 appears to be too faint for an RRL (Table C.1). The resolved Bound candidate companion of HM Aql (Fig. C.1) is a low mass dwarf of spectral type K7V located at a large projected separation of $14.7 \mathrm{kau}$.

\subsubsection{EN CMi}

The absolute magnitude of EN CMi in the $G$ band $\left(M_{G}=5.34\right)$ is too faint for an RRL, although the light curve presented in Vivas et al. (2004) is similar in shape to that of a $P=0.540 \mathrm{~d}$ RRab type pulsator. It is located in a region of low extinction. Assuming that the GDR2 parallax of EN CMi is correct, its resolved companion is of comparable brightness and spectral type $\mathrm{K} 1.5 \mathrm{~V}$.

\subsubsection{NQ Cyg}

NQ Cyg is classified as an eclipsing binary by Coughlin et al. (2014). Little information is present in the literature on this variable, but its absolute magnitude is too faint to be an RRL. Taichi Kato $^{5}$ proposed from its ASAS-SN light curve (Shappee et al. 2014; Kochanek et al. 2017) that it is an R-type object (close eclipsing binary with strong reflection lighting effects) with a period $P=0.311592 \mathrm{~d}$.

We identified a low mass Bound candidate companion (Fig. C.1) of spectral type K9V located at a projected separation of $19.4 \mathrm{kau}$ (Table C.1). Due to their fast proper motion $(\mu>$ $12 \mathrm{mas} \mathrm{a}^{-1}$ ) and almost perfect PM vector alignment (within $0.35^{\circ}$ ), the probability of a chance association is negligible.

\subsubsection{V1391 Cyg}

V1391 Cyg is classified as an eclipsing binary in the catalog by Coughlin et al. (2014). We detected a resolved Bound candidate companion at a projected separation of $19 \mathrm{kau}$ (Fig. C.1), with a probable spectral type of M1.5V (Table C.1) based on its $G$-band absolute magnitude. We note, however, that the Gaia colors $G_{\mathrm{BP}}$ and $G_{\mathrm{RP}}$ are not mutually consistent with the $G$ magnitude and are possibly biased.

\subsubsection{V2121 Cyg}

V2121 Cyg (43 Cyg, HD 195069) was identified as a possible RRL from Hipparcos photometry (Perryman et al. 1997). However, Henry et al. (2005) established that V2121 Cyg is one of the brightest $\gamma$ Dor non-radial pulsators $\left(m_{G}=5.63\right)$. Fekel et al. (2003) proposed that it may be a spectroscopic binary star. It was recently studied in detail by Zwintz et al. (2017) using photometric time series from the BRITE-Constellation nano-satellites. This is a main sequence F-type star with an effective temperature of around $7000 \mathrm{~K}$. We detected a gravitationally bound companion to V2121 Cyg, Gaia DR2 2084032103278208640 (Fig. C.1), approximately 6.6 magnitudes fainter than the primary at visible wavelengths $\left(m_{G}=12.27\right)$. The large parallax $(\varpi \approx 25.6$ mas $)$ and very fast proper motion of the two stars $\left(\mu \approx 90\right.$ mas a $\left.^{-1}\right)$ excludes a chance association. The companion has an effective temperature $T_{\text {eff }}=3700 \pm 100 \mathrm{~K}$ and a radius $R=0.50 \pm 0.02 R_{\odot}$, which correspond to a $\mathrm{M} 2 \mathrm{~V}$ spectral type (Table C.1). It exhibits a significant X-ray emission (source 1RXS J202704.9+492216; Haakonsen \& Rutledge 2009, see also Boller et al. 2016).

\footnotetext{
5 http://ooruri.kusastro.kyoto-u.ac.jp/mailarchive/ vsnet-chat/8021
} 


\subsubsection{UU Dor}

UU Dor is identified as an LMC detached eclipsing binary in the OGLE-III catalog (Graczyk et al. 2011). However, its GDR2 parallax implies that it is a Galactic variable, of combined spectral type F6V. Its resolved Bound candidate companion (Fig. C.1) is a low mass dwarf of spectral type $\mathrm{K} 3 \mathrm{~V}$ (Table C.1).

\subsubsection{IW Lib}

IW Lib is listed in the McDonald et al. (2012) catalog, where it does not show infrared excess. It is classified by Pojmanski (2002) as an eclipsing contact binary with an orbital period of $1.78 \mathrm{~d}$. It has been automatically classified as a $\gamma$ Dor non-radial pulsator by Dubath et al. (2011) and more probably as a W UMa eclipsing binary (with a probability of 0.29 ) by Richards et al. (2012), who mostly exclude the possibility of an RRL. The spectral type of the wide companion is $\mathrm{K} 3 \mathrm{~V}$ (Table C.1), with an effective temperature $T_{\text {eff }}=4800 \pm 200 \mathrm{~K}$ and a radius $R=0.71 \pm 0.03 R_{\odot}$. It is $5.3 \mathrm{mag}$ fainter than the primary in the $G$ band.

\subsubsection{AZ Men}

AZ Men is classified as an RRc pulsator with a period of $0.318 \mathrm{~d}$ (Otero 2008); however, its absolute magnitude $M_{G}=+3.7$ is inconsistent with this class. Its resolved companion (Fig. C.2) has an effective temperature $T_{\text {eff }}=4900 \pm 200 \mathrm{~K}$ and a radius $R=0.63 \pm 0.04 R_{\odot}$. These parameters were derived using the surface brightness-color relations from Kervella et al. (2004) and the GDR2 parallax, and match those of a K3V dwarf (Table C.1).

\subsubsection{V1171 Oph}

This star is likely a contact eclipsing binary, of combined spectral type F8V with a mean effective temperature of $T_{\text {eff }} \approx$ $6200 \mathrm{~K}$. Its resolved companion (Fig. C.2) is a red dwarf of spectral type K1V.

\subsubsection{V1330 Sgr}

Although its absolute $G$ magnitude is inconsistent with a classification as an RRL $\left(M_{G}=+6.9\right), \mathrm{V} 1330 \mathrm{Sgr}$ is listed as an RRab pulsator with $P=0.597$ d by Soszyński et al. (2011), showing the Blazhko effect. It is located in the direction of the Galactic Bulge, close to Baade's field (Plaut 1973), but its GDR2 parallax $\left(\varpi_{\mathrm{G} 2}=1.23 \pm 0.22\right.$ mas $)$ places it at a significantly shorter distance. Assuming the GDR2 parallax is correct, its resolved companion (Fig. C.2) is an MOV red dwarf, located at a projected physical separation of $24.7 \mathrm{kau}$.

\subsubsection{V1382 Sgr}

Soszyński et al. (2014) classified V1382 Sgr as an RRab type pulsator with a period of $P=0.493 \mathrm{~d}$ with Blazhko effect (Collinge et al. 2006), located in the direction of the Bulge. However, this is in principle excluded by its very faint absolute magnitude $M_{G}=+7.3$, unless the GDR2 parallax is incorrect. Assuming the GDR2 parallax is correct, its resolved common proper motion companion is an $\mathrm{M} 2 \mathrm{~V}$ red dwarf (Fig. C.2), approximately 2 mag fainter than the primary in the $G$ band, and located at a projected physical separation of $11.6 \mathrm{kau}$ (Table C.1). It should be noted that the crowding in this region is high and could have caused a bias in the GDR2 parallax estimate.

\subsubsection{V2248 Sgr}

V2248 Sgr is classified as a W UMa eclipsing binary by Antipin et al. (2005), but it is classified as a DSCT/EC/ESD in the ASAS-3 database (Pojmanski 2002) and an RR Lyrae pulsator in the GCVS (Samus et al. 2009). Its period is $P=0.315 \mathrm{~d}$ in the GCVS and $0.158 \mathrm{~d}$ (half of the former) in the ASAS-3 database. The wide companion (Fig. C.2) has an effective temperature $T_{\text {eff }}=4700 \pm 200 \mathrm{~K}$ and a radius $R=0.73 \pm 0.03 R_{\odot}$. These properties correspond to a K3.5V spectral type.

\subsubsection{V3166 Sgr}

The field around V3166 Sgr shows two candidate bound companions, making it a visual triple star. The classification as a RRL of this variable star in the GCVS is unlikely to be correct considering its absolute $G$-band magnitude (Table C.1). The close companion (at a separation of $1.66^{\prime \prime}$ ), considering its absolute magnitude and assuming it is on the main sequence, is most probably a late K-type dwarf. The more distant companion has $T_{\text {eff }}=3500 \pm 100 \mathrm{~K}$ and $R=0.79 \pm 0.03 R_{\odot}$, which possibly corresponds to an unusual post-main sequence, "inflated" M1 spectral type dwarf. It could result from the evolution of a $\approx 0.6 M_{\odot}$ star, of mid-K spectral type on the main sequence, at an age of $\approx 10 \mathrm{Ga}$ (Bressan et al. 2012).

\subsubsection{HR Sco}

With an absolute $G$ band magnitude of $M_{G}=+10.2$, HR Sco is likely a very low mass main sequence star of spectral type M3V. The candidate resolved companion of HR Sco (Fig. C.3) is located at a projected separation of $20 \mathrm{kau}$, for a spectral type of M5V (Table C.1).

\subsubsection{EN TrA}

EN TrA is a binary RV Tauri type variable (Van Winckel et al. 1999). We detected a strong PMa $\left(\Delta_{\mathrm{G} 2}=11.7\right.$; Paper I) that confirms the presence of an orbiting companion. We did not detect any resolved common proper motion candidate in the GDR2.

\section{Conclusion}

We detected a number of new candidate companions of Cepheids and RR Lyrae stars, either from their signature on the proper motion of the targets (Paper I) or the similarity of their parallax and proper motion to those of the targets (present Paper II).

Classical Cepheids have long been known to have a high binarity fraction, and our survey of the PM anomalies of bright and nearby CCs indicates that their binarity fraction is likely higher than $80 \%$ (Paper I), with a significant fraction of triple (e.g., SY Nor, AW Per, W Sgr, V0350 Sgr) or quadruple systems (e.g., $\delta$ Cep, SY Nor). The resolved systems that we discovered or confirmed in the present paper (Paper II) provide interesting fiducial references for the modeling of the evolutionary state of the corresponding $\mathrm{CC}$ primaries. They also enable the validation of the GDR2 parallaxes of their CC companions that may be affected by a specific uncertainty due to their photometric and color variability.

The gravitationally bound candidates of RRLs that we present in Table B.1 are all new discoveries, and provide important constraints on the evolutionary state of their pulsating companions. In Paper I we find that a small but significant fraction $(\approx 7 \%)$ of the nearby RRLs shows indications of binarity. The relatively large number of stars in our sample that were 
P. Kervella et al.: Multiplicity of Galactic Cepheids and RR Lyrae stars from Gaia DR2. II.

misclassified as RRLs is a call for a thorough revision of the RRL catalogs using the Gaia data, and in particular the parallax, to reclassify the other types of variables.

Acknowledgements. We thank the organizers of the MIAPP workshop "The extragalactic distance scale in the Gaia era" held on 11 June-6 July 2018 in Garching (Germany); it provided a place for very useful exchanges that enhanced the work presented in the present paper. We thank Dr. Richard I. Anderson for the interesting discussion on Polaris and $\delta$ Cep that led to improvements in this paper. This work has made use of data from the European Space Agency (ESA) mission Gaia (http://www. cosmos.esa.int/gaia), processed by the Gaia Data Processing and Analysis Consortium (DPAC, http://www. cosmos.esa. int/web/gaia/dpac/consortium). Funding for the DPAC has been provided by national institutions, in particular the institutions participating in the Gaia Multilateral Agreement. The authors acknowledge the support of the French Agence Nationale de la Recherche (ANR), under grant ANR-15-CE31-001201 (project UnlockCepheids). The research leading to these results has received funding from the European Research Council (ERC) under the European Union's Horizon 2020 research and innovation program (grant agreement No. 695099). Support was provided to NRE by the Chandra X-ray Center NASA Contract NAS8-03060. This study was funded by the NKFIH K-115709 grant of the Hungarian National Research and Innovation Office. W.G. and G.P. gratefully acknowledge financial support for this work from the BASAL Centro de Astrofisica y Tecnologias Afines (CATA) AFB-170002. W.G. acknowledges financial support from the Millenium Institute of Astrophysics (MAS) of the Iniciativa Cientifica Milenio del Ministerio de Economia, Fomento y Turismo de Chile, project IC120009. We acknowledge support from the IdP II 2015000264 grant of the Polish Ministry of Science and Higher Education. We acknowledge the past financial support to this research program of the "Programme National de Physique Stellaire" (PNPS) of CNRS/INSU, France. This research has made use of Astropy, a community-developed core Python package for Astronomy (Astropy Collaboration 2013, 2018). This research has made use of the International Variable Star Index (VSX) database, operated at AAVSO, Cambridge, Massachusetts, USA. This research was supported by the Munich Institute for Astro- and Particle Physics (MIAPP) of the DFG cluster of excellence "Origin and Structure of the Universe". We used the SIMBAD and VIZIER databases and catalog access tool at the CDS, Strasbourg (France), and NASA's Astrophysics Data System Bibliographic Services. The original description of the VizieR service was published in Ochsenbein et al. (2000). The Digitized Sky Surveys were produced at the Space Telescope Science Institute under US Government grant NAG W2166. The images of these surveys are based on photographic data obtained using the Oschin Schmidt Telescope on Palomar Mountain and the UK Schmidt Telescope. The plates were processed into the present compressed digital form with the permission of these institutions. This research has made use of the Washington Double Star Catalog maintained at the US Naval Observatory. This publication makes use of data products from the Two Micron All Sky Survey, which is a joint project of the University of Massachusetts and the Infrared Processing and Analysis Center/California Institute of Technology, funded by the National Aeronautics and Space Administration and the National Science Foundation.

\section{References}

Abt, H. A., \& Levy, S. G. 1978, PSAP, 90, 188

Anderson, R. I. 2018, A\&A, 611, L7

Anderson, R. I., Eyer, L., \& Mowlavi, N. 2013, MNRAS, 434, 2238

Anderson, R. I., Sahlmann, J., Holl, B., et al. 2015, ApJ, 804, 144

Anderson, R. I., Casertano, S., Riess, A. G., et al. 2016, ApJS, 226, 18

Antipin, S. V., Pastukhova, E. N., \& Samus, N. N. 2005, Inf. Bull. Variable Stars, 5613

Astropy Collaboration (Robitaille, T. P., et al.) 2013, A\&A, 558, A33

Astropy Collaboration (Price-Whelan, A. M., et al.) 2018, AJ, 156, 123

Babel, J., Burki, G., Mayor, M., Waelkens, C., \& Chmielewski, Y. 1989, A\&A, 216, 125

Benedict, G. F., McArthur, B., Chappell, D. W., et al. 1999, AJ, 118, 1086

Benedict, G. F., McArthur, B. E., Franz, O. G., Wasserman, L. H., \& Henry, T. J. 2000, AJ, 120, 1106

Benedict, G. F., McArthur, B. E., Fredrick, L. W., et al. 2002, AJ, 124, 1695

Benedict, G. F., McArthur, B. E., Feast, M. W., et al. 2007, AJ, 133, 1810

Berdnikov, L. N., Dambis, A. K., \& Vozyakova, O. V. 2000, A\&AS, 143, 211

Bernacca, P. L., \& Perinotto, M. 1970, Contributi dell'Osservatorio Astrofisica dell'Universita di Padova in Asiago, 239, 1

Bersier, D., Burki, G., Mayor, M., \& Duquennoy, A. 1994, A\&AS, 108, 25

Bohm-Vitense, E., \& Proffitt, C. 1985, ApJ, 296, 175

Bohm-Vitense, E., Clark, M., Cottrell, P. L., \& Wallerstein, G. 1990, AJ, 99, 353

Bohm-Vitense, E., Evans, N. R., Carpenter, K., et al. 1997, AJ, 114, 1176

Böhm-Vitense, E., Evans, N. R., Carpenter, K., et al. 1998, ApJ, 505, 903
Boller, T., Freyberg, M. J., Trümper, J., et al. 2016, A\&A, 588, A103 Bond, H. E., Nelan, E. P., Remage Evans, N., Schaefer, G. H., \& Harmer, D. 2018, ApJ, 853, 55

Bonnardeau, M., \& Hambsch, F.-J. 2015, Inf. Bull. Variable Stars, 6132

Borgniet, S., Kervella, P., Mérand, A., et al. 2018, A\&A, submitted

Bramich, D. M., Alsubai, K. A., Arellano Ferro, A., et al. 2014, Inf. Bull. Variable Stars, 6106, 1

Bressan, A., Marigo, P., Girardi, L., et al. 2012, MNRAS, 427, 127

Chen, X., de Grijs, R., \& Deng, L. 2015, MNRAS, 446, 1268

Chen, X., de Grijs, R., \& Deng, L. 2017, MNRAS, 464, 1119

Clementini, G., Ripepi, V., Molinaro, R., et al. 2019, A\&A, 622, A60

Collinge, M. J., Sumi, T., \& Fabrycky, D. 2006, ApJ, 651, 197

Coughlin, J. L., Thompson, S. E., Bryson, S. T., et al. 2014, AJ, 147, 119

Cutri, R. M., Skrutskie, M. F., van Dyk, S., et al. 2003, 2MASS All Sky Catalog of Point Sources

Cutri, R. M., Wright, E. L., Conrow, T., et al. 2012, VizieR Online Data Catalog, II/311

Cuypers, J., Aerts, C., De Cat, P., et al. 2009, A\&A, 499, 967

Drake, A. J., Catelan, M., Djorgovski, S. G., et al. 2013, ApJ, 763, 32

Dubath, P., Rimoldini, L., Süveges, M., et al. 2011, MNRAS, 414, 2602

Duchêne, G., \& Kraus, A. 2013, ARA\&A, 51, 269

Efremov, Y. N. 1964, Peremennye Zvezdy, 15, 242

Eggen, O. J. 1983, AJ, 88, 379

Engle, S. G., Guinan, E. F., Harper, G. M., et al. 2017, ApJ, 838, 67

Engle, S. G., Guinan, E. F., \& Harmanec, P. 2018, Res. Notes AAS, 2, 126

Evans, N. R. 1988, ApJS, 66, 343

Evans, N. R. 1990, PSAP, 102, 551

Evans, N. R. 1991, ApJ, 372, 597

Evans, N. R. 1992a, ApJ, 385, 680

Evans, N. R. 1992b, ApJ, 384, 220

Evans, N. R. 1994, ApJ, 436, 273

Evans, N. R. 1995, ApJ, 445, 393

Evans, N. R. 2000, AJ, 119, 3050

Evans, N. R., \& Butler, J. 1993, PSAP, 105, 915

Evans, N. R., \& Lyons, R. 1986, AJ, 92, 436

Evans, N. R., \& Udalski, A. 1994, AJ, 108, 653

Evans, N. R., Welch, D. L., Scarfe, C. D., \& Teays, T. J. 1990, AJ, 99, 1598

Evans, N. R., Welch, D. L., Slovak, M. H., Barnes, III., T. G., \& Moffett, T. J. 1993, AJ, 106, 1599

Evans, N. R., Massa, D., \& Teays, T. J. 1994, AJ, 108, 2251

Evans, N. R., Carpenter, K., Robinson, R., et al. 1999, ApJ, 524, 379

Evans, N. R., Vinko, J., \& Wahlgren, G. M. 2000, AJ, 120, 407

Evans, N. R., Sasselov, D. D., \& Short, C. I. 2002, ApJ, 567, 1121

Evans, N. R., Carpenter, K. G., Robinson, R., Kienzle, F., \& Dekas, A. E. 2005 AJ, 130, 789

Evans, N. R., Schaefer, G. H., Bond, H. E., et al. 2008, AJ, 136, 1137

Evans, N. R., Bond, H. E., Schaefer, G. H., et al. 2013, AJ, 146, 93

Evans, N. R., Pillitteri, I., Wolk, S., et al. 2014, ApJ, 785, L25

Evans, N. R., Berdnikov, L., Lauer, J., et al. 2015, AJ, 150, 13

Evans, N. R., Bond, H. E., Schaefer, G. H., et al. 2016a, AJ, 151, 129

Evans, N. R., Pillitteri, I., Wolk, S., et al. 2016b, AJ, 151, 108

Evans, N. R., Karovska, M., Bond, H. E., et al. 2018a, ApJ, 863, 187

Evans, D. W., Riello, M., De Angeli, F., et al. 2018b, A\&A, 616, A4

Evans, N. R., Proffitt, C., Carpenter, K. G., et al. 2018c, ApJ, 866, 30

Feast, M. W., Laney, C. D., Kinman, T. D., van Leeuwen, F., \& Whitelock, P. A 2008, MNRAS, 386, 2115

Fekel, F. C., Warner, P. B., \& Kaye, A. B. 2003, AJ, 125, 2196

Fernie, J. D. 1990, ApJS, 72, 153

Fernie, J. D. 1994, ApJ, 429, 844

Fernley, J., \& Barnes, T. G. 1997, A\&AS, 125, 313

Fouqué, P., Arriagada, P., Storm, J., et al. 2007, A\&A, 476, 73

Freedman, W. 2013, The Hubble Constant to $1 \%$ ? STAGE 4: Calibrating the RR Lyrae PL relation at H-Band using HST and Gaia Parallax Stars, HST Proposal

Gaia Collaboration (Brown, A. G. A., et al.) 2016, A\&A, 595, A2

Gaia Collaboration (Clementini, G., et al.) 2017, A\&A, 605, A79

Gaia Collaboration (Babusiaux, C., et al.) 2018, A\&A, 616, A10

Gallenne, A., Monnier, J. D., Mérand, A., et al. 2013, A\&A, 552, A21

Gallenne, A., Mérand, A., Kervella, P., et al. 2014a, A\&A, 561, L3

Gallenne, A., Kervella, P., Mérand, A., et al. 2014b, A\&A, 567, A60

Gallenne, A., Mérand, A., Kervella, P., et al. 2015, A\&A, 579, A68

Gallenne, A., Mérand, A., Kervella, P., et al. 2016, MNRAS, 461, 1451

Gallenne, A., Kervella, P., Evans, N. R., et al. 2018a, ApJ, 867, 121

Gallenne, A., Kervella, P., Mérand, A., et al. 2018b, ApJ, submitted

Gavrilchenko, T., Klein, C. R., Bloom, J. S., \& Richards, J. W. 2014, MNRAS, 441,715

Gillet, D. 2013, A\&A, 554, A46

Gontcharov, G. A. 2017, Astron. Lett., 43, 472 
Gorynya, N. A., Rastorguev, A. S., \& Samus, N. N. 1996a, Astron. Lett., 22, 33 Gorynya, N. A., Samus', N. N., Rastorguev, A. S., \& Sachkov, M. E. 1996b, Astron. Lett., 22, 175

Graczyk, D., Soszyński, I., Poleski, R., et al. 2011, Acta Astron., 61, 103 Gravity Collaboration (Abuter, R., et al.) 2017, A\&A, 602, A94

Green, G. M., Schlafly, E. F., Finkbeiner, D., et al. 2018, MNRAS, 478, 651

Greer, P. A., Payne, S. G., Norton, A. J., et al. 2017, A\&A, 607, A11

Griffin, R. F. 2016, The Observatory, 136, 209

Groenewegen, M. A. T. 2008, A\&A, 488, 25

Groenewegen, M. A. T. 2013, A\&A, 550, A70

Groenewegen, M. A. T. 2018, A\&A, 619, A8

Haakonsen, C. B., \& Rutledge, R. E. 2009, ApJSS, 184, 138

Hajdu, G., Jurcsik, J., \& Sodor, A. 2009, Inf. Bull. Variable Stars, 5882, 1

Havlen, R. J. 1972, A\&A, 16, 252

Henry, G. W., Fekel, F. C., \& Henry, S. M. 2005, AJ, 129, 2815

Hoyle, F., Shanks, T., \& Tanvir, N. R. 2003, MNRAS, 345, 269

Huziak, R. 2006, J. R. Astron. Soc. Can., 100, 130

Imbert, M. 1996, A\&AS, 116, 497

Iorio, G., Belokurov, V., Erkal, D., et al. 2018, MNRAS, 474, 2142

Irwin, J. B. 1955, Mon. Notes Astron. Soc. S. Afr., 14, 38

Jaschek, M., \& Jaschek, C. 1960, PSAP, 72, 500

Jeffers, H. M., van den Bos, W. H., \& Greeby, F. M. 1963, Index Catalogue of Visual Double Stars, 1961.0 (Publications of the Lick Observatory, Mount Hamilton: University of California, Lick Observatory)

Jordi, C., Gebran, M., Carrasco, J. M., et al. 2010, A\&A, 523, A48

Kamper, K. W. 1996, JRASC, 90, 140

Kemper, E. 1982, AJ, 87, 1395

Kervella, P., Thévenin, F., Di Folco, E., \& Ségransan, D. 2004, A\&A, 426, 297

Kervella, P., Mérand, A., Szabados, L., et al. 2012, A\&A, 541, A18

Kervella, P., Bond, H. E., Cracraft, M., et al. 2014, A\&A, 572, A7

Kervella, P., Thévenin, F., \& Lovis, C. 2017a, A\&A, 598, L7

Kervella, P., Trahin, B., Bond, H. E., et al. 2017b, A\&A, 600, A127

Kervella, P., Gallenne, A., Evans, N. R., et al. 2019, A\&A, 623, A116 (Paper I)

Kharchenko, N. V., Piskunov, A. E., Schilbach, E., Röser, S., \& Scholz, R. D 2016, A\&A, 585, A101

Kiss, L. L., Szatmary, K., Gal, J., \& Kaszas, G. 1995, Inf. Bull. Variable Stars, 4205, 1

Klagyivik, P., \& Szabados, L. 2009, A\&A, 504, 959

Kochanek, C. S., Shappee, B. J., Stanek, K. Z., et al. 2017, PSAP, 129, 104502

Kovtyukh, V. V., \& Andrievsky, S. M. 1999, A\&A, 350, L55

Kovtyukh, V. V., Soubiran, C., Luck, R. E., et al. 2008, MNRAS, 389, 1336

Kurochkin, N. E. 1958, Peremennye Zvezdy, 12, 277

LaCluyzé, A., Smith, H. A., Gill, E. M., et al. 2004, AJ, 127, 1653

Lallement, R., Capitanio, L., Ruiz-Dern, L., et al. 2018, A\&A, 616, A132

Liška, J., Skarka, M., Mikulášek, Z., Zejda, M., \& Chrastina, M. 2016a, A\&A, 589, A94

Liška, J., Skarka, M., Zejda, M., Mikulášek, Z., \& de Villiers, S. N. 2016b, MNRAS, 459, 4360

Lloyd Evans, T. 1982, MNRAS, 199, 925

Luck, R. E., Andrievsky, S. M., Kovtyukh, V. V., Gieren, W., \& Graczyk, D. 2011, AJ, 142, 51

Madore, B. F. 1977, MNRAS, 178, 505

Magakian, T. Y. 2003, A\&A, 399, 141

Maintz, G. 2005, A\&A, 442, 381

Majaess, D., Turner, D. G., \& Gieren, W. 2012a, MNRAS, 421, 1040

Majaess, D., Turner, D. G., Gallo, L., et al. 2012b, ApJ, 753, 144

Majaess, D., Turner, D., \& Gieren, W. 2012c, ApJ, 747, 145

Majaess, D., Turner, D., Gieren, W., Balam, D., \& Lane, D. 2012d, ApJ, 748, L9

Majaess, D., Carraro, G., Moni Bidin, C., et al. 2013, A\&A, 560, A22

Marengo, M., Evans, N. R., Barmby, P., et al. 2010, ApJ, 725, 2392

Mason, B. D., Wycoff, G. L., Hartkopf, W. I., Douglass, G. G., \& Worley, C. E. 2001, AJ, 122, 3466

Massa, D., \& Evans, N. R. 2008, MNRAS, 383, 139

Matthews, L. D., Marengo, M., Evans, N. R., \& Bono, G. 2012, ApJ, 744, 53

McDonald, I., Zijlstra, A. A., \& Boyer, M. L. 2012, MNRAS, 427, 343

Mérand, A., Aufdenberg, J. P., Kervella, P., et al. 2007, ApJ, 664, 1093

Mérand, A., Kervella, P., Breitfelder, J., et al. 2015, A\&A, 584, A80

Mermilliod, J. C., Mayor, M., \& Burki, G. 1987, A\&AS, 70, 389

Morgan, B. L., Beddoes, D. R., Scaddan, R. J., \& Dainty, J. C. 1978, MNRAS, 183,701

Morgan, S. M., Wahl, J. N., \& Wieckhorst, R. M. 2007, MNRAS, 374, 1421

Muraveva, T., Delgado, H. E., Clementini, G., Sarro, L. M., \& Garofalo, A. 2018, MNRAS, 481, 1195

Ochsenbein, F., Bauer, P., \& Marcout, J. 2000, A\&AS, 143, 23

Oh, S., Price-Whelan, A. M., Hogg, D. W., Morton, T. D., \& Spergel, D. N. 2017 , AJ, 153, 257

Olah, K., \& Szeidl, B. 1978, Commun. Konkoly Obs. Hungary, 71, 1
Otero, S. A. 2008, Open Eur. J. Variable Stars, 0093, 1

Peña, J. H., \& Peniche, R. 2004, Rev. Mex. Astron. Astrofis., 40, 99 Pecaut, M. J., \& Mamajek, E. E. 2013, ApJS, 208, 9

Pecaut, M. J., Mamajek, E. E., \& Bubar, E. J. 2012, ApJ, 746, 154

Pel, J. W. 1978, A\&A, 62, 75

Perryman, M. A. C., Lindegren, L., Kovalevsky, J., et al. 1997, A\&A, 323, L49

Petterson, O. K. L., Cottrell, P. L., \& Albrow, M. D. 2004, MNRAS, 350, 95

Pickering, E. C., \& Wendell, O. C. 1899, ApJ, 9, 179

Pietrzyński, G., Thompson, I. B., Gieren, W., et al. 2012, Nature, 484, 75

Pietrzyński, G., Graczyk, D., Gieren, W., et al. 2013, Nature, 495, 76

Plaut, L. 1973, A\&A, 26, 317

Pojmanski, G. 2002, Acta Astron., 52, 397

Price-Whelan, A. M., Oh, S., \& Spergel, D. N. 2017, ArXiv e-prints [arXiv:1709.03532]

Proust, D., Ochsenbein, F., \& Pettersen, B. R. 1981, A\&AS, 44, 179

Richards, J. W., Starr, D. L., Miller, A. A., et al. 2012, ApJSS, 203, 32

Riess, A. G., Macri, L. M., Hoffmann, S. L., et al. 2016, ApJ, 826, 56

Rimoldini, L., Eyer, L., Mowlavi, N., et al. 2018, All-Sky RR Lyrae Stars in the Gaia Data, 6, 101

Roberts, Jr., L. C. 2011, MNRAS, 413, 1200

Sahlmann, J., Lazorenko, P. F., Ségransan, D., et al. 2013, A\&A, 556, A133

Samus, N. N., Durlevich, O. V., \& Kazarovets, E. V. 2009, VizieR Online Data Catalog, 1, 2025

Samus, N. N., Kazarovets, E. V., Durlevich, O. V., Kireeva, N. N., \& Pastukhova, E. N. 2017, Astron. Rep., 61, 80

Scholz, R.-D. 2016, A\&A, 587, A51

Shappee, B. J., Prieto, J. L., Grupe, D., et al. 2014, ApJ, 788, 48

Skarka, M. 2013, A\&A, 549, A101

Skarka, M. 2014a, A\&A, 562, A90

Skarka, M. 2014b, MNRAS, 445, 1584

Skarka, M., Liška, J., Auer, R. F., et al. 2016, A\&A, 592, A144

Skrutskie, M. F., Cutri, R. M., Stiening, R., et al. 2006, AJ, 131, 1163

Slovak, M. H., van Citters, G. W., \& Barnes, III., T. G. 1979, PSAP, 91, 840

Smith, H. A. 2006, Mem. Soc. Astron. It., 77, 492

Smolec, R., Pietrzyński, G., Graczyk, D., et al. 2013, MNRAS, 428, 3034

Sódor, Á., Jurcsik, J., Szeidl, B., et al. 2011, MNRAS, 411, 1585

Soszyński, I., Dziembowski, W. A., Udalski, A., et al. 2011, Acta Astron., 61, 1

Soszyński, I., Udalski, A., Szymański, M. K., et al. 2014, Acta Astron., 64, 177

Szabados, L. 1989, Commun. Konkoly Obs. Hungary, 94, 1

Szabados, L. 1991, Commun. Konkoly Obs. Hungary, 96, 123

Szabados, L. 1996, A\&A, 311, 189

Szabados, L. 2003, Inf. Bull. Variable Stars, 5394

Szczygieł, D. M., Pojmański, G., \& Pilecki, B. 2009, Acta Astron., 59, 137

Szeidl, B., Olah, K., \& Mizser, A. 1986, Commun. Konkoly Obs. Hungary, 89, 57

Tammann, G. A., Sandage, A., \& Reindl, B. 2003, A\&A, 404, 423

ten Brummelaar, T. A., McAlister, H. A., Ridgway, S. T., et al. 2005, ApJ, 628, 453

Tokovinin, A. 2017, MNRAS, 468, 3461

Turner, D. G. 1976, AJ, 81, 1125

Turner, D. G. 1992, AJ, 104, 1865

Turner, D. G. 2010, Ap\&SS, 326, 219

Turner, D. G., \& Evans, N. R. 1984, ApJ, 283, 254

Turner, D. G., Mandushev, G. I., \& Welch, G. A. 1997, AJ, 113, 2104

Turner, D. G., Pedreros, M. H., \& Walker, A. R. 1998, AJ, 115, 1958

Usenko, I. A., \& Klochkova, V. G. 2008, MNRAS, 387, L1

van Leeuwen, F., ed. 2007a, in Hipparcos, The New Reduction of the Raw Data (Berlin: Springer Science \& Business Media), Astrophysics and Space Science Library, 350

van Leeuwen, F. 2007b, A\&A, 474, 653

van Leeuwen, F., Feast, M. W., Whitelock, P. A., \& Laney, C. D. 2007, MNRAS, 379,723

Van Winckel, H., Waelkens, C., Fernie, J. D., \& Waters, L. B. F. M. 1999, A\&A, 343,202

Vivas, A. K., Zinn, R., Abad, C., et al. 2004, AJ, 127, 1158

Wade, R. A., Donley, J., Fried, R., White, R. E., \& Saha, A. 1999, AJ, 118, 2442

Wahlgren, G. M., \& Evans, N. R. 1998, A\&A, 332, L33

Watson, C. L., Henden, A. A., \& Price, A. 2006, Soc. Astron. Sci. Annu. Symp., 25,47

Welch, D. L., \& Evans, N. R. 1989, AJ, 97, 1153

Welch, D. L., Evans, N. R., Lyons, R. W., et al. 1987, PSAP, 99, 610

Westerlund, B. 1961, PSAP, 73, 72

Wils, P., Lloyd, C., \& Bernhard, K. 2006, MNRAS, 368, 1757

Wright, E. L., Eisenhardt, P. R. M., Mainzer, A. K., et al. 2010, AJ, 140, 1868

Zwintz, K., Van Reeth, T., Tkachenko, A., et al. 2017, A\&A, 608, A103 
P. Kervella et al.: Multiplicity of Galactic Cepheids and RR Lyrae stars from Gaia DR2. II.

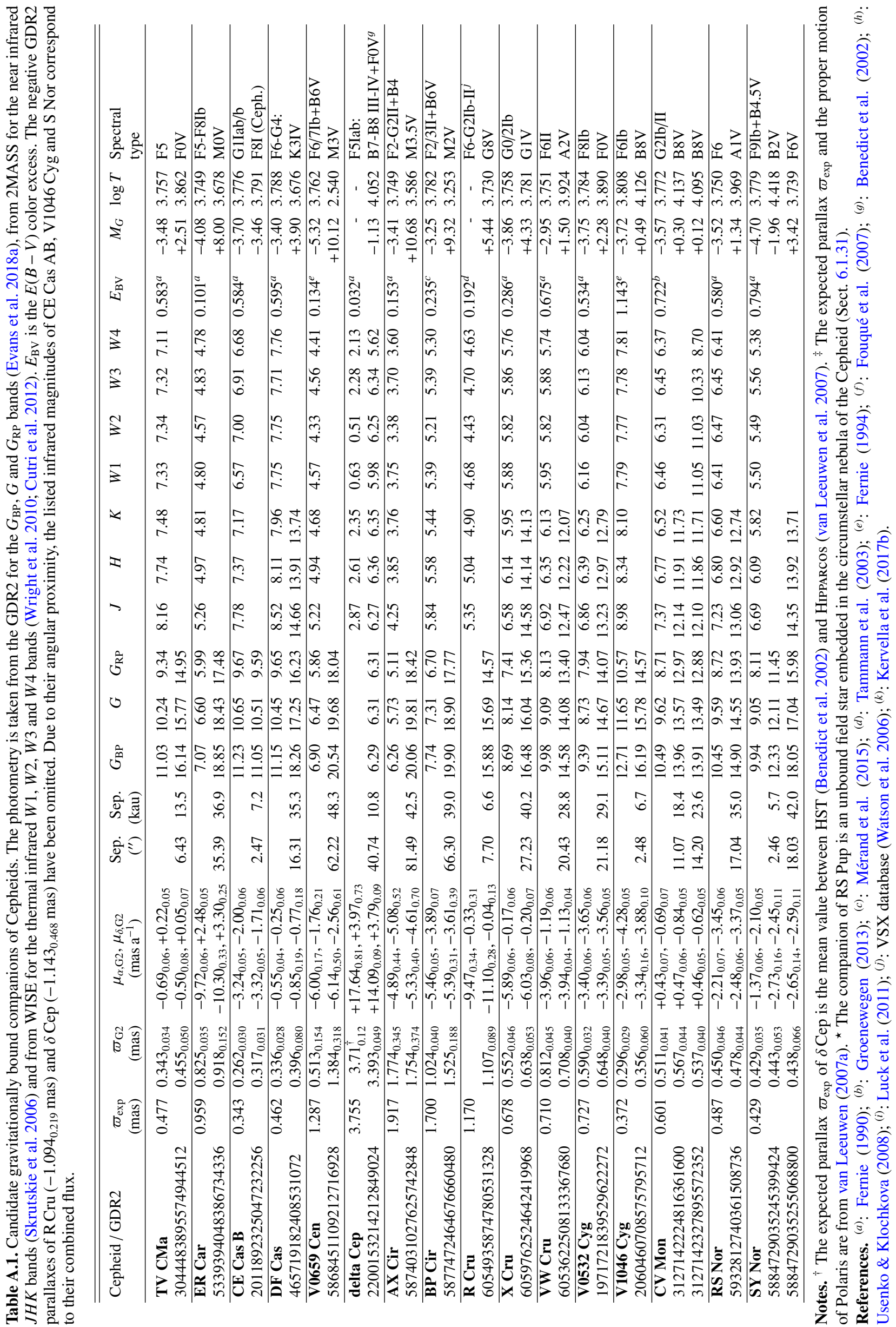




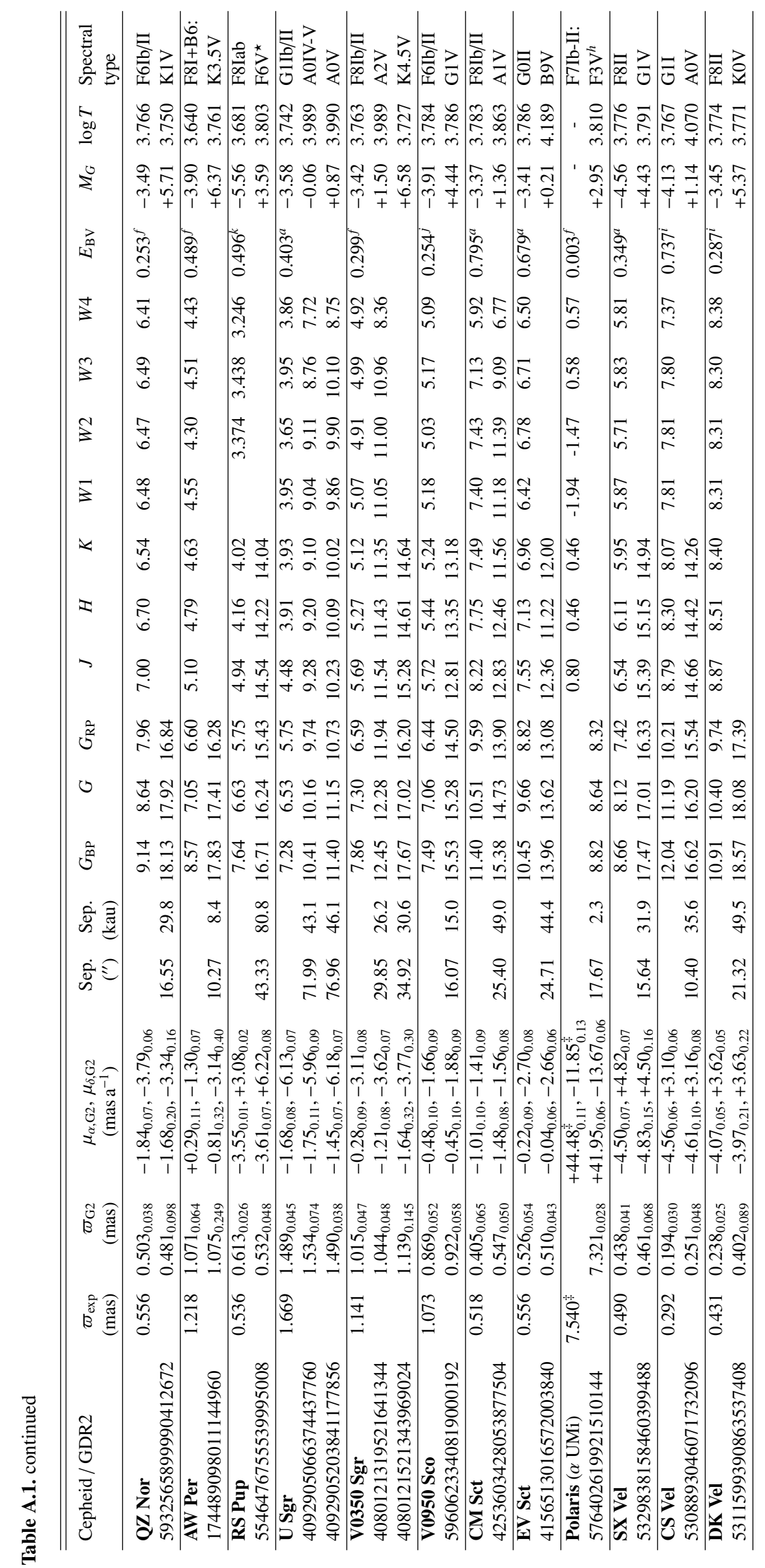


P. Kervella et al.: Multiplicity of Galactic Cepheids and RR Lyrae stars from Gaia DR2. II.

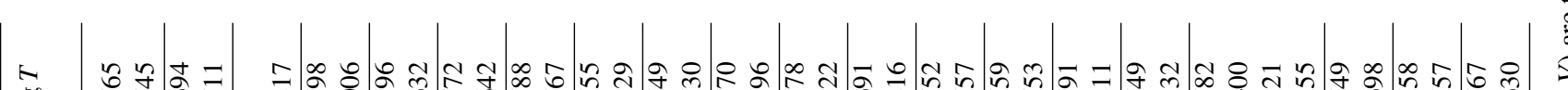

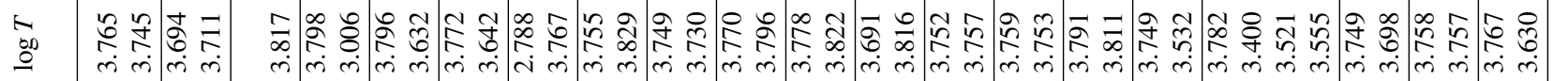

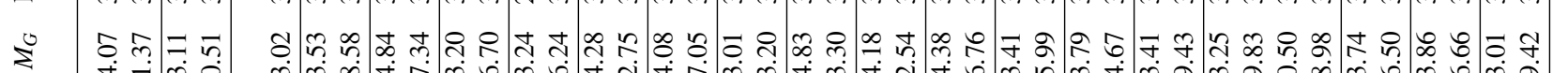

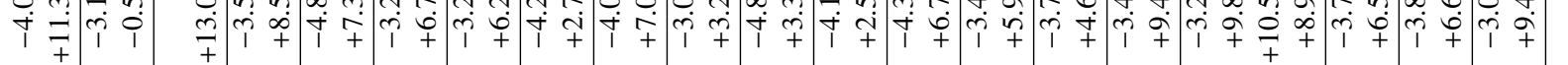

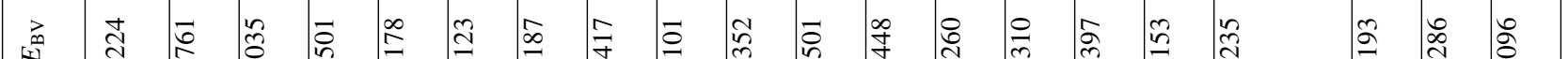

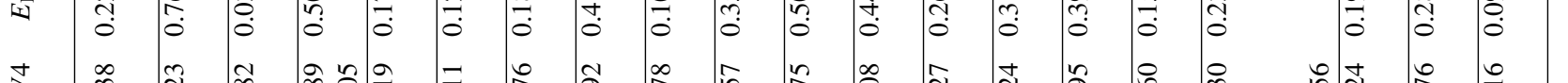

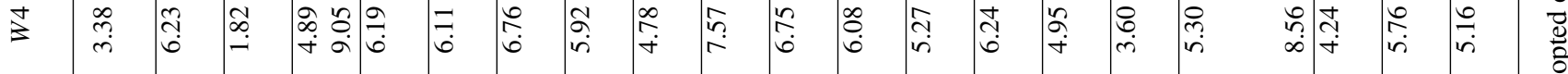

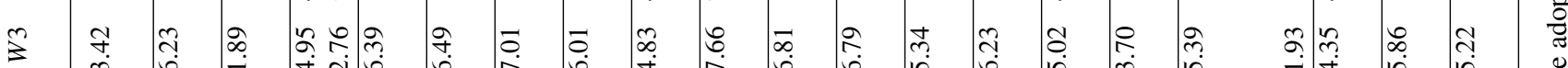

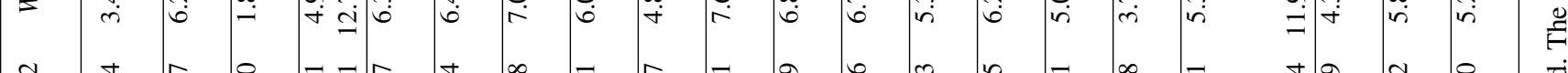

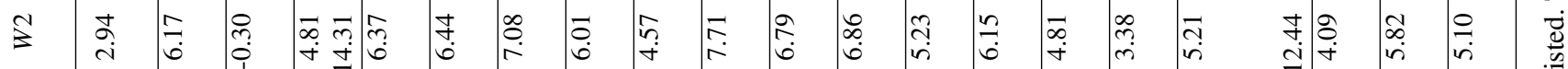

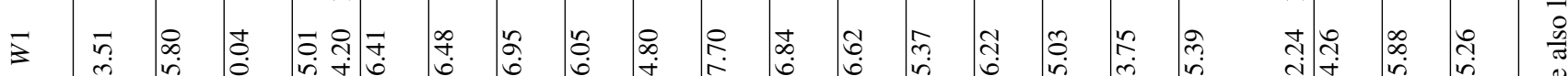

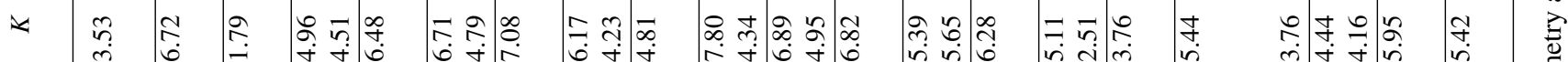

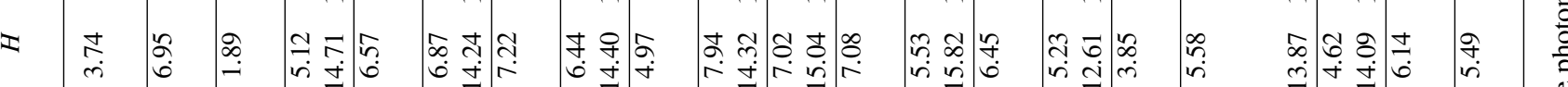

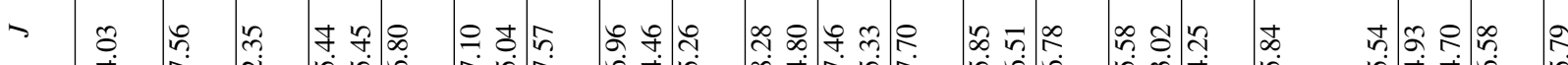

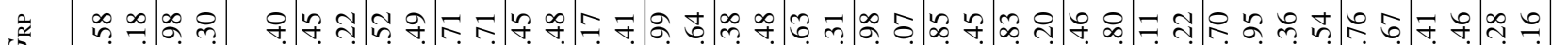

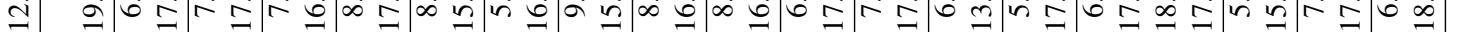

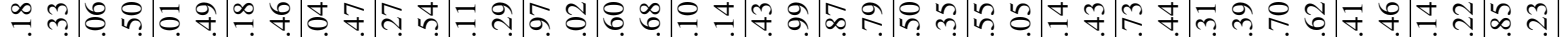

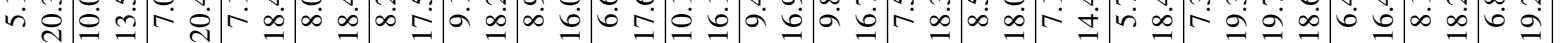
商

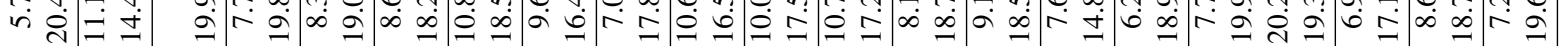

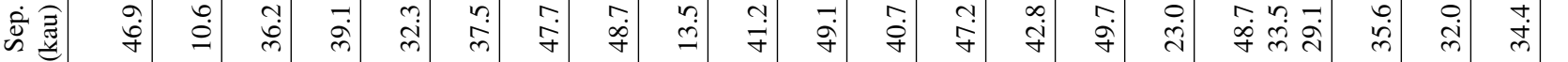

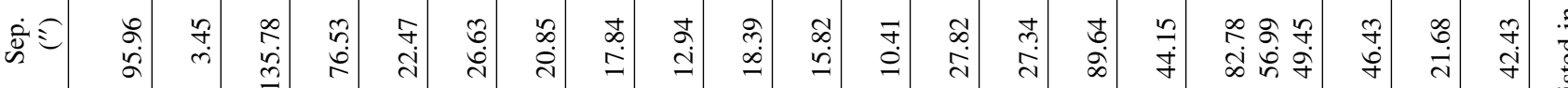

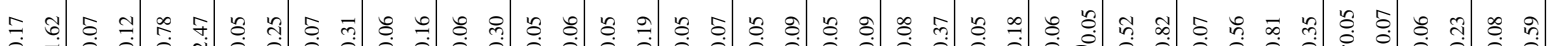

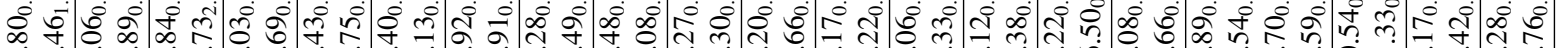
б

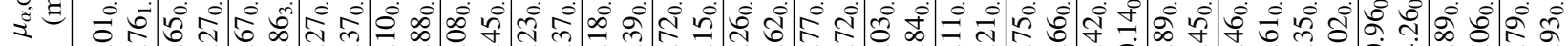

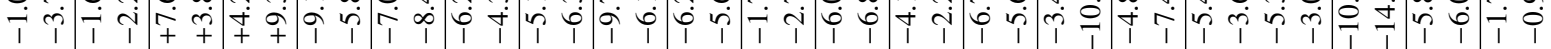

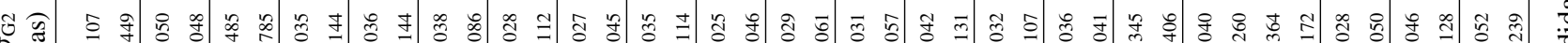
m0

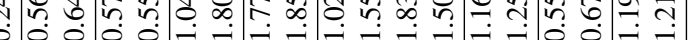

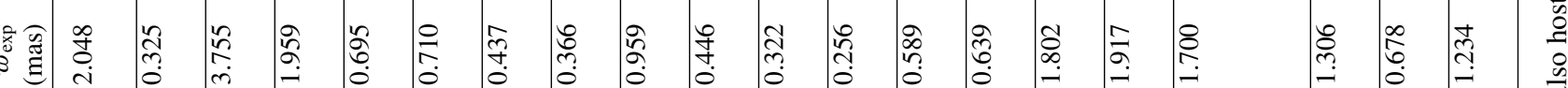

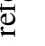
$\stackrel{5}{ \pm}$ $\frac{\pi}{3}$ i 


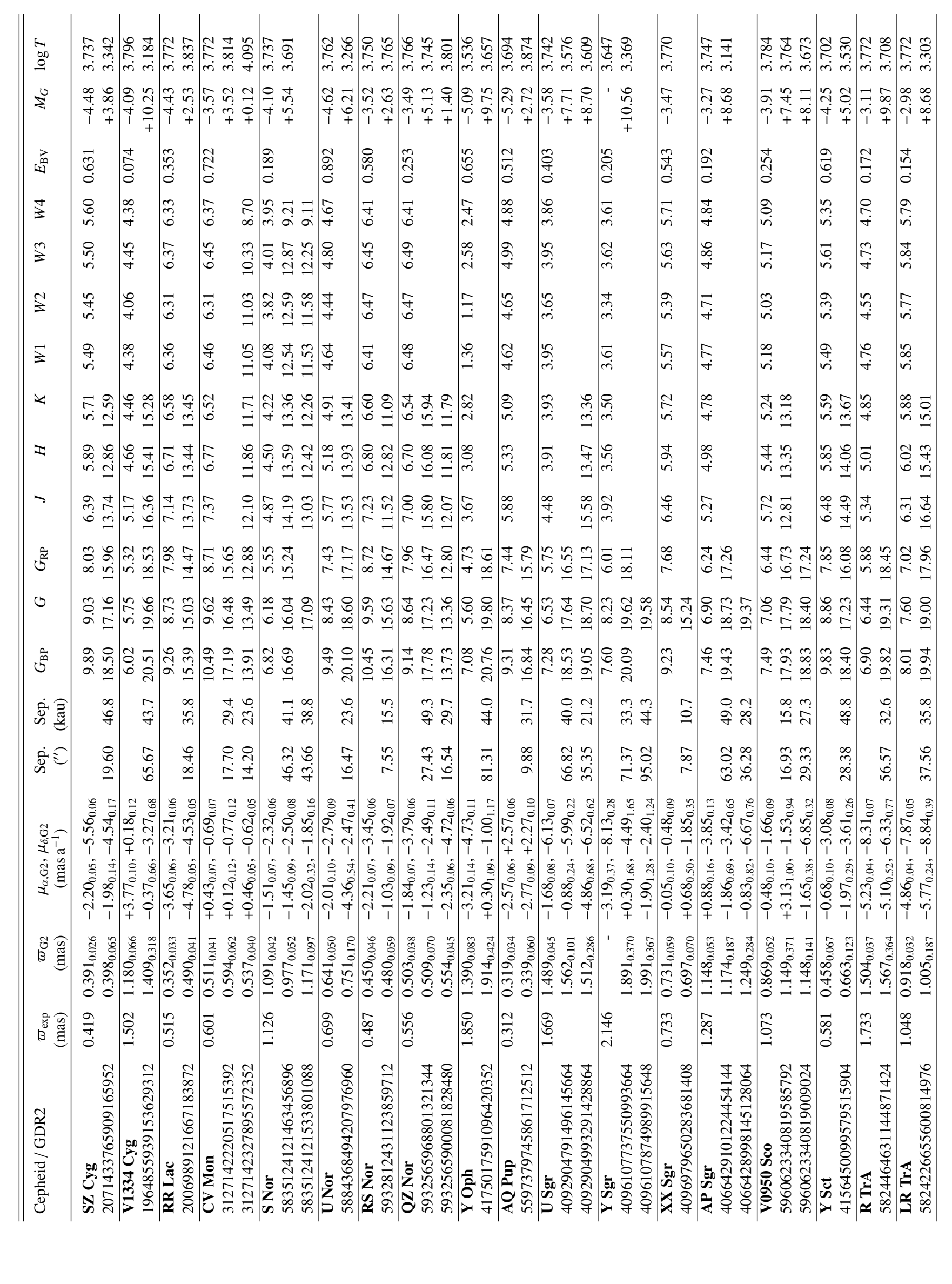


P. Kervella et al.: Multiplicity of Galactic Cepheids and RR Lyrae stars from Gaia DR2. II.

\section{A.2. Field charts}

The fields surrounding the CCs with detected Bound candidate companions, and a selection of CCs with Near candidate companions are presented in Figs. A.1-A.8. $\delta$ Cep and Polaris are discussed separately in Sects. 6.1.9 and 6.1.42. The target position is shown with a magenta + symbol, and the tested field stars (with a parallax within $\pm 15 \%$ of that of the target) are represented with cyan $\times$ symbols. The identified Near candidates are represented in orange, the LowV candidates are shown in red, and the Bound candidates are indicated with a yellow star symbol. Their proper motion vectors, and that of the target, are shown. The GDR2 proper motion vector $\boldsymbol{\mu}_{\mathrm{DR} 2}$ is shown in magenta for each target star. When available, the mean proper motion vector of the target $\mu_{\mathrm{HG}}$ estimated from the HIPPARcos and GDR2 positions (see Paper I) is also displayed in light green. The expected parallax of the target is indicated in the upper left corner of each panel, together with the GDR2 value. For CCs, this is the renormalized Leavitt law parallax from the catalog by Berdnikov et al. (2000; see Sect. 3.1). The background images are taken from the DSS2-Red.
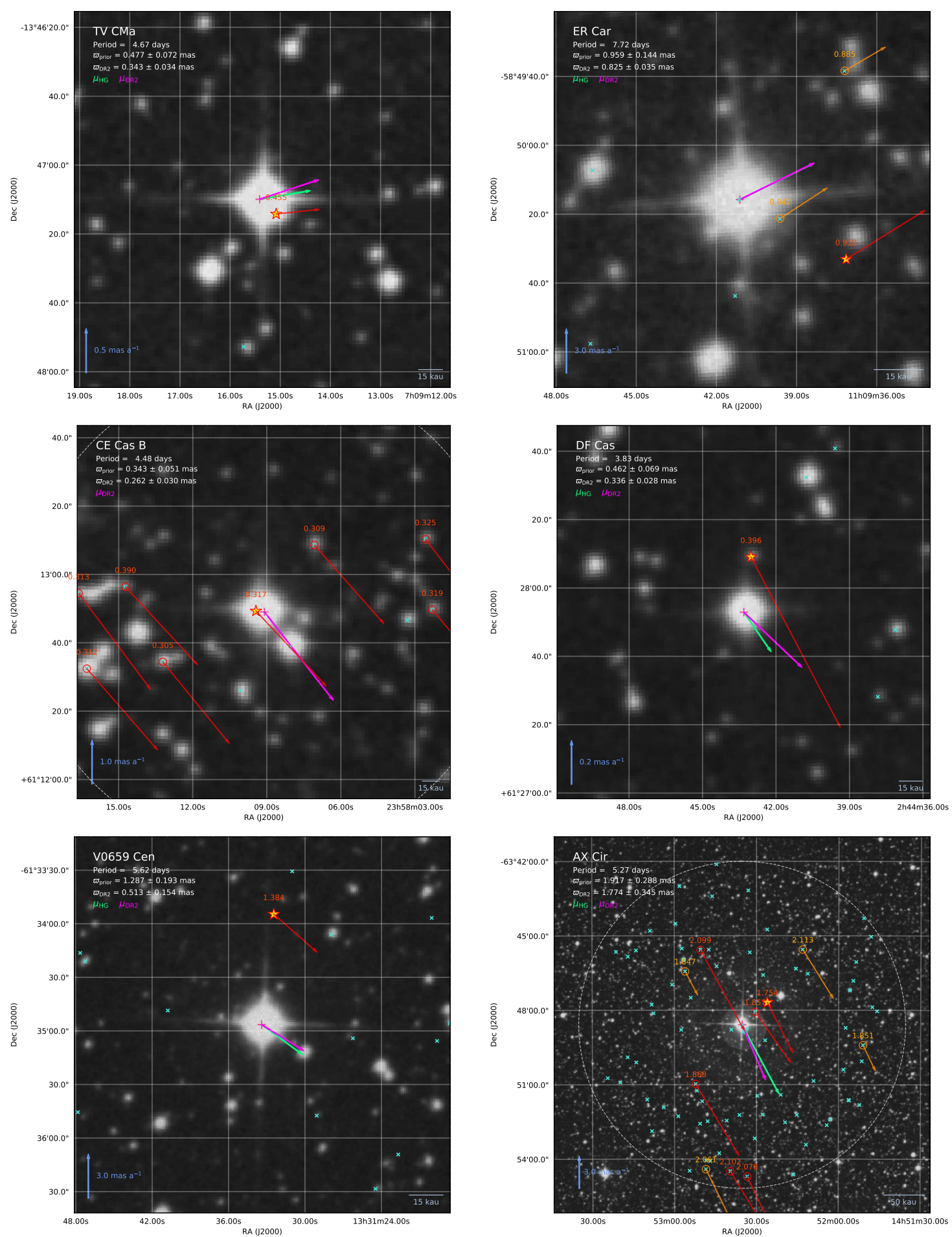

Fig. A.1. Field charts for Cepheids with Bound candidate companions: TV CMa, ER Car, CE Cas, DF Cas, V0659 Cen and AX Cir. 
A\&A 623, A117 (2019)
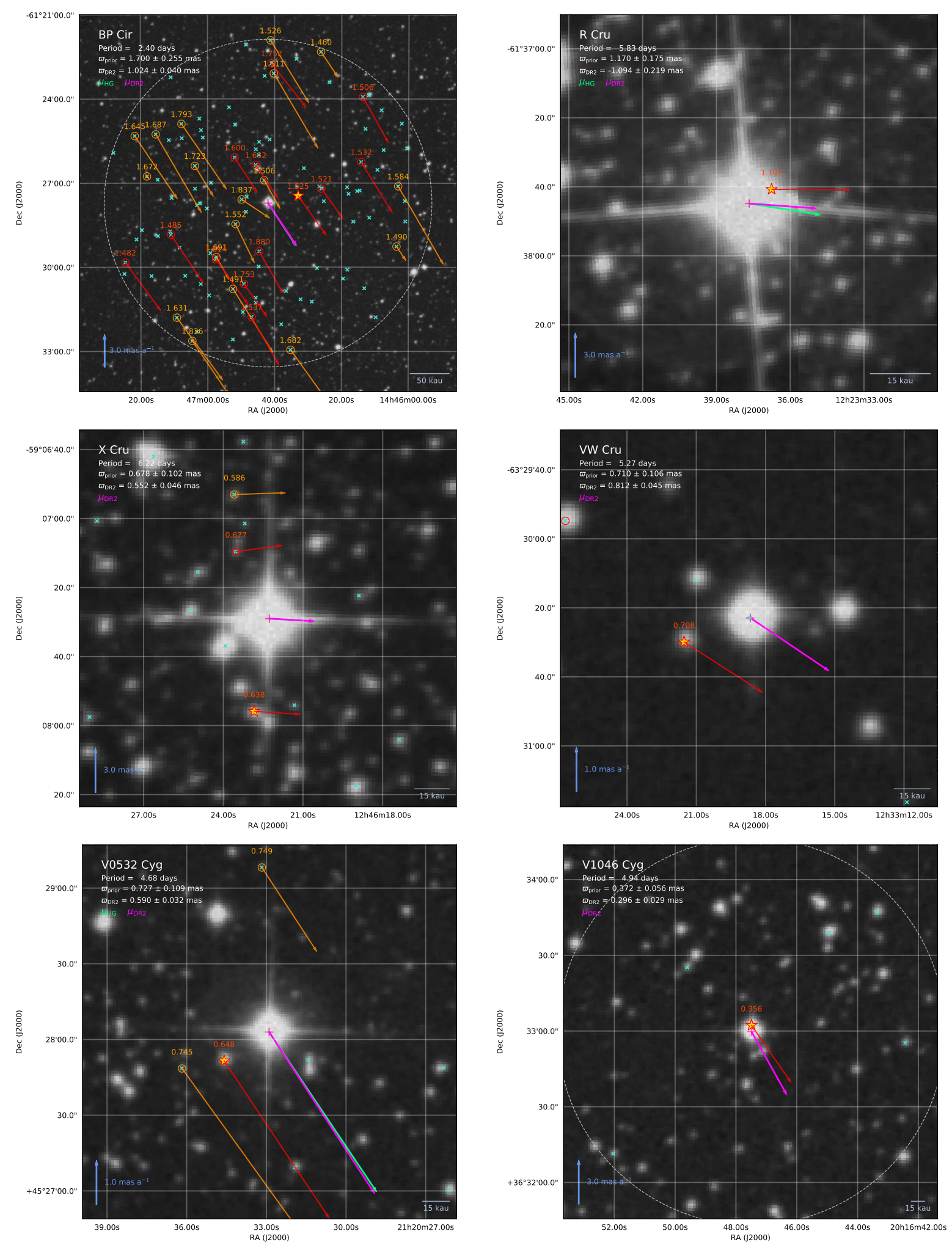

Fig. A.2. Same as Fig. A.1 for BP Cir, R Cru, X Cru, VW Cru, V0532 Cyg and V1046 Cyg. 
P. Kervella et al.: Multiplicity of Galactic Cepheids and RR Lyrae stars from Gaia DR2. II.
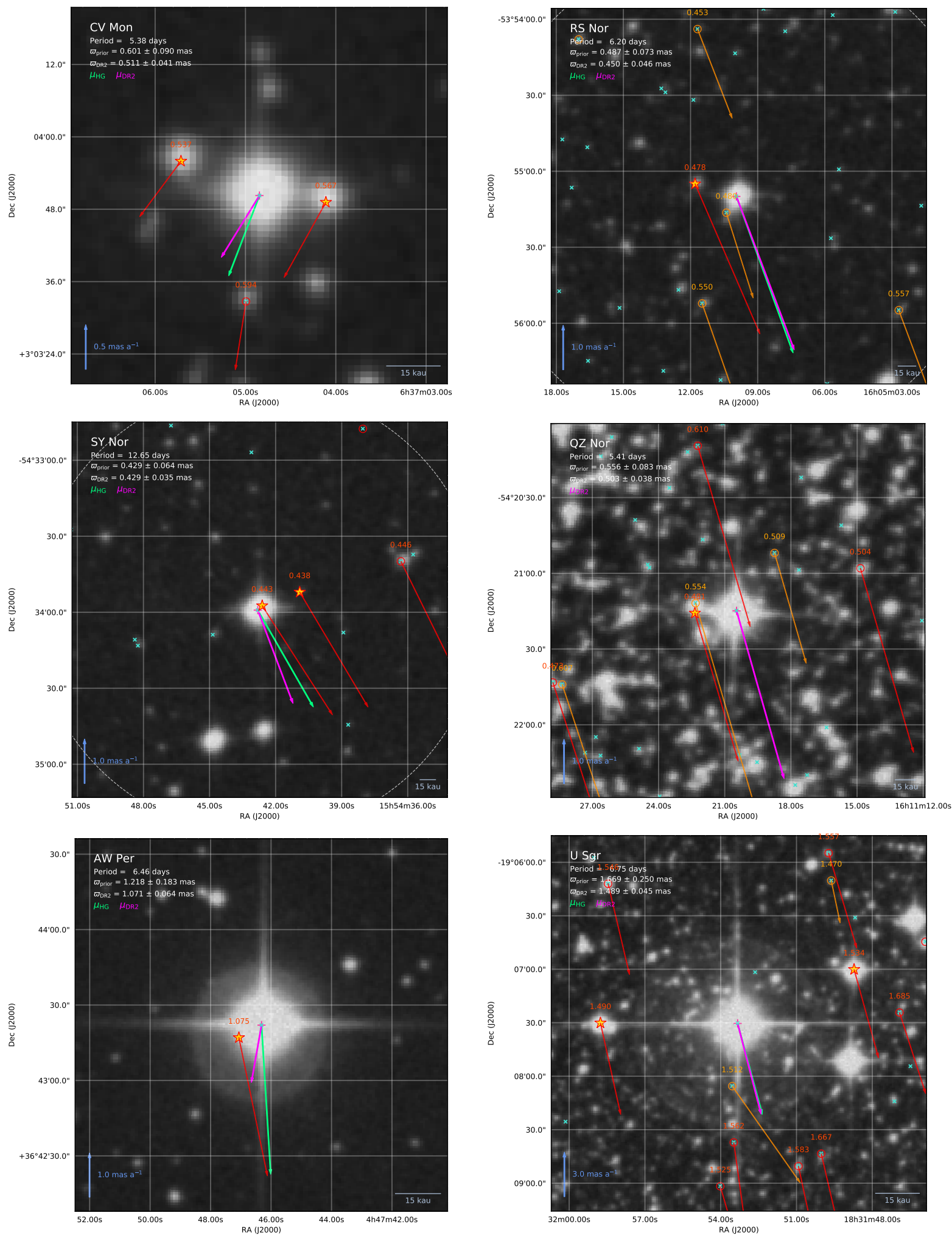

Fig. A.3. Same as Fig. A.2 for CV Mon, RS Nor, SY Nor, QZ Nor, AW Per and U Sgr. 
A\&A 623, A117 (2019)
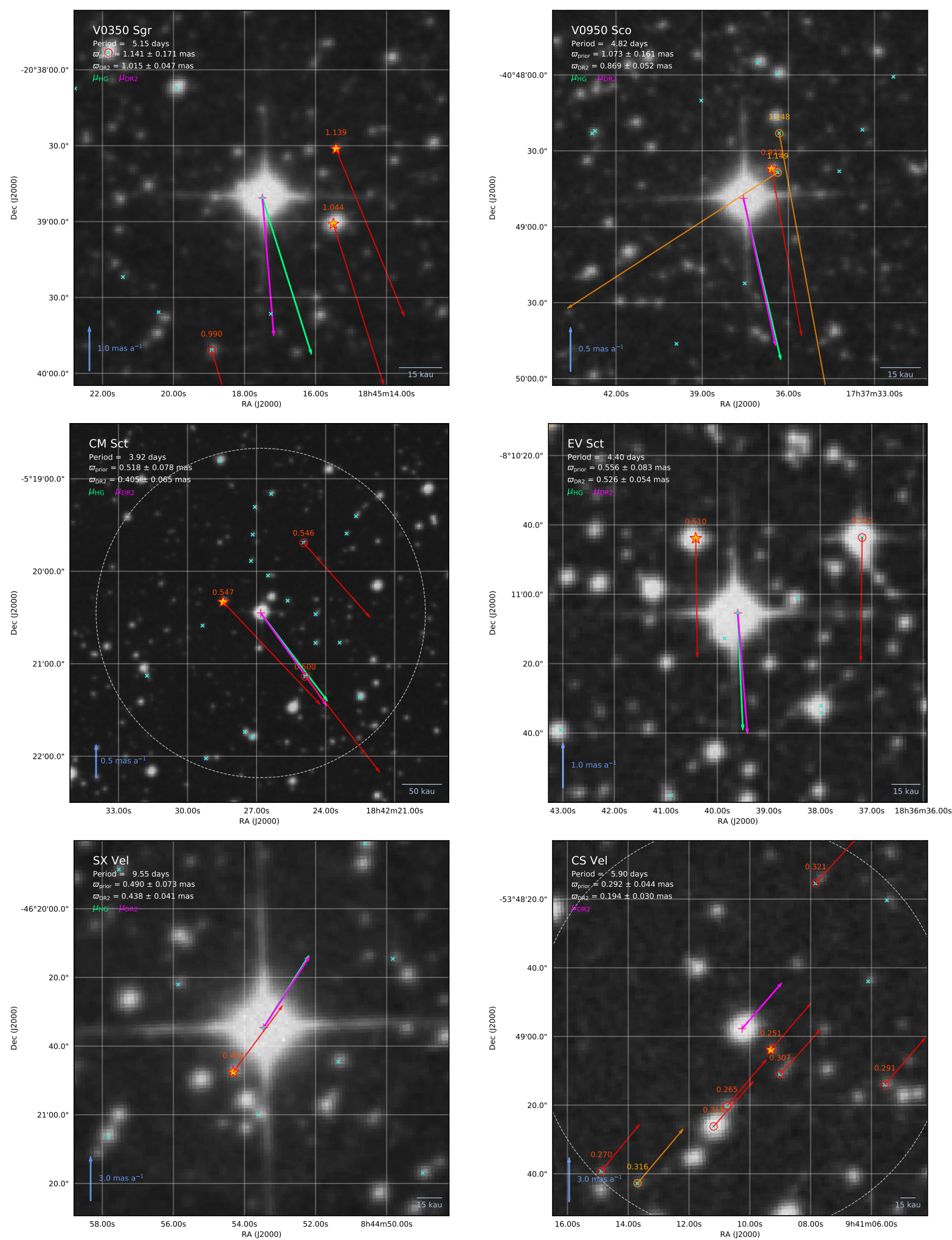

Fig. A.4. Same as Fig. A.3 for V0350 Sgr, V0950 Sco, CM Sct, EV Sct, SX Vel and CS Vel. 
P. Kervella et al.: Multiplicity of Galactic Cepheids and RR Lyrae stars from Gaia DR2. II.

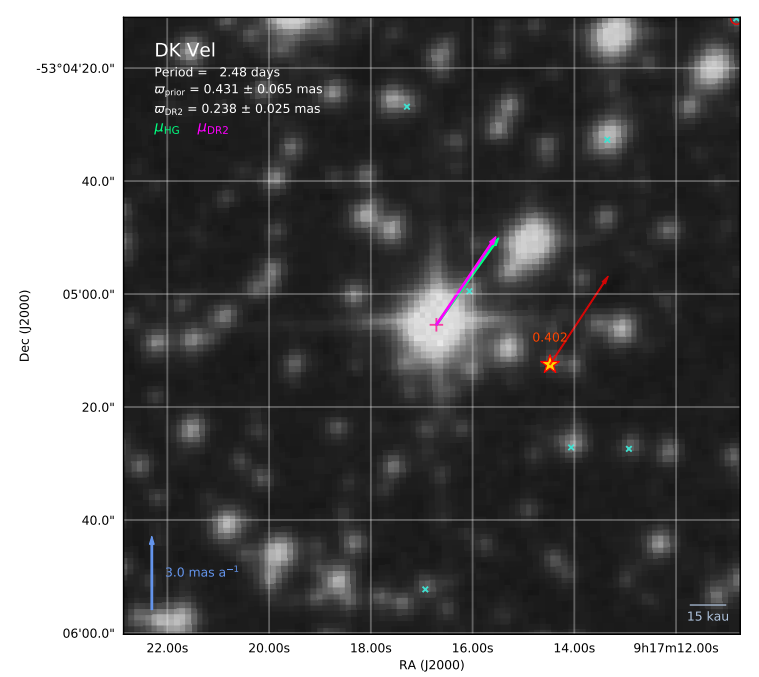

Fig. A.5. Same as Fig. A.4 for DK Vel. 

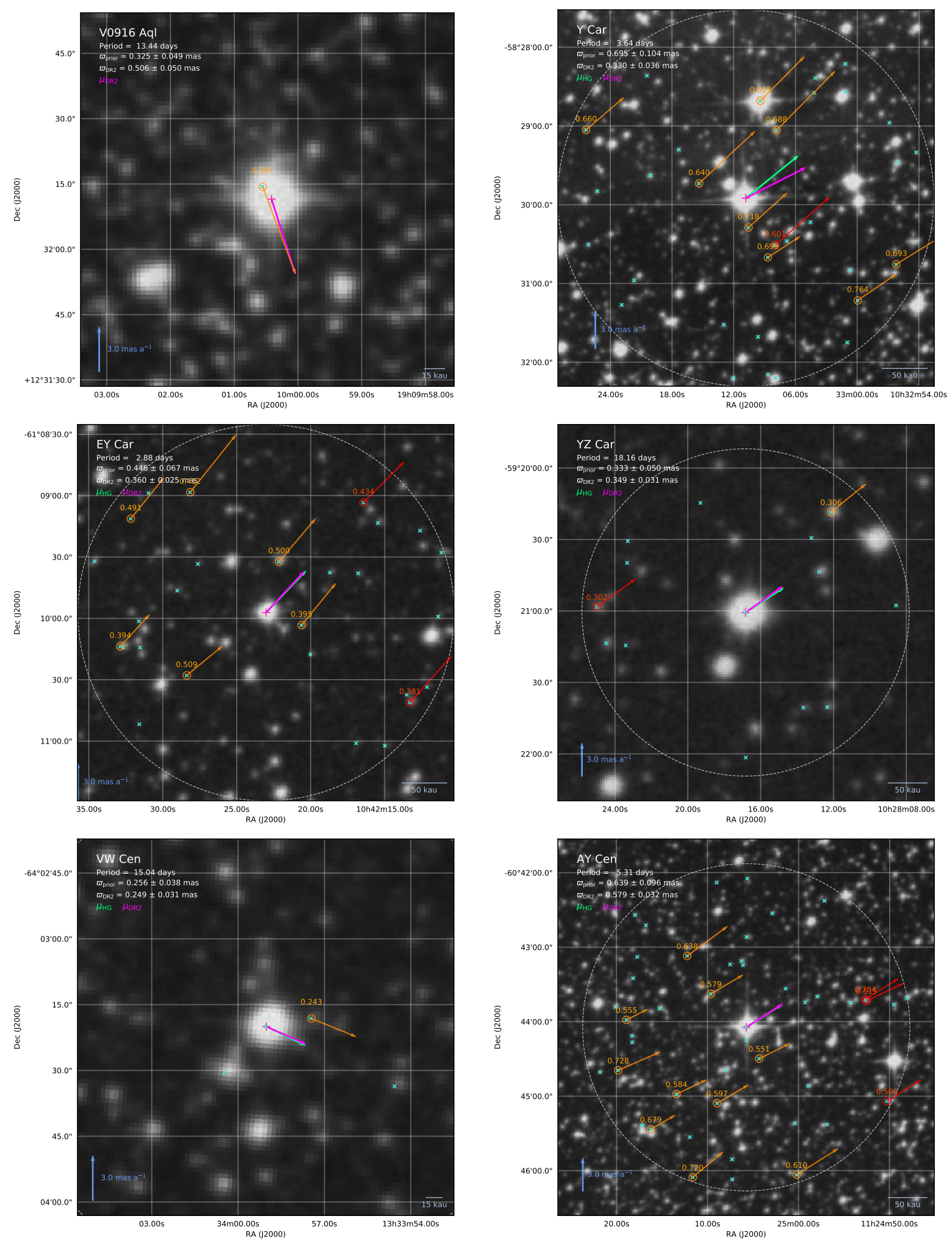

Fig. A.6. Field charts for Cepheids with Near candidate companions: V0916 Aql, Y Car, EY Car, YZ Car, VW Cen and AY Cen. 
P. Kervella et al.: Multiplicity of Galactic Cepheids and RR Lyrae stars from Gaia DR2. II.
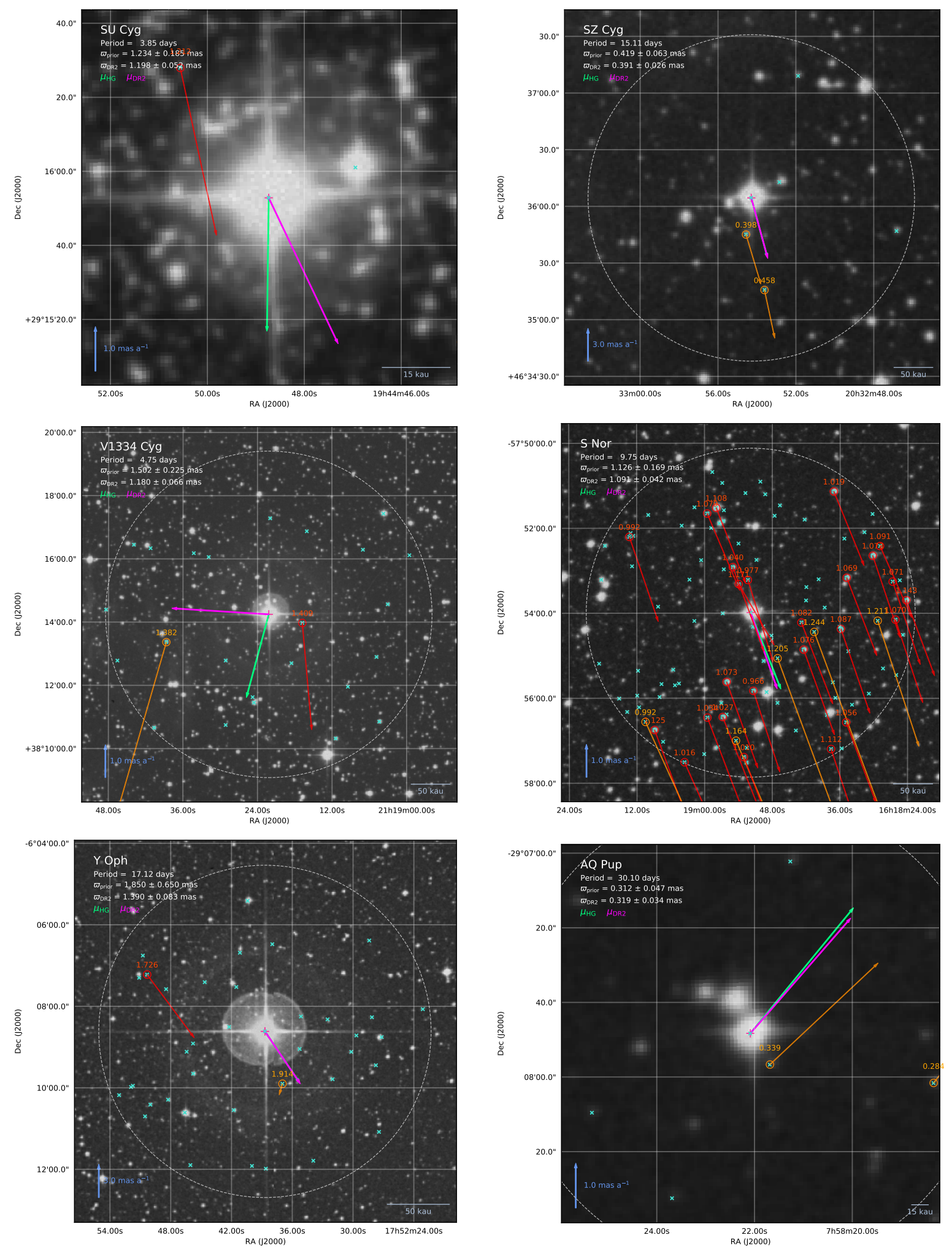

Fig. A.7. Same as Fig. A.6 for SU Cyg, SZ Cyg, V1334 Cyg, S Nor, Y Oph and AQ Pup. 
A\&A 623, A117 (2019)
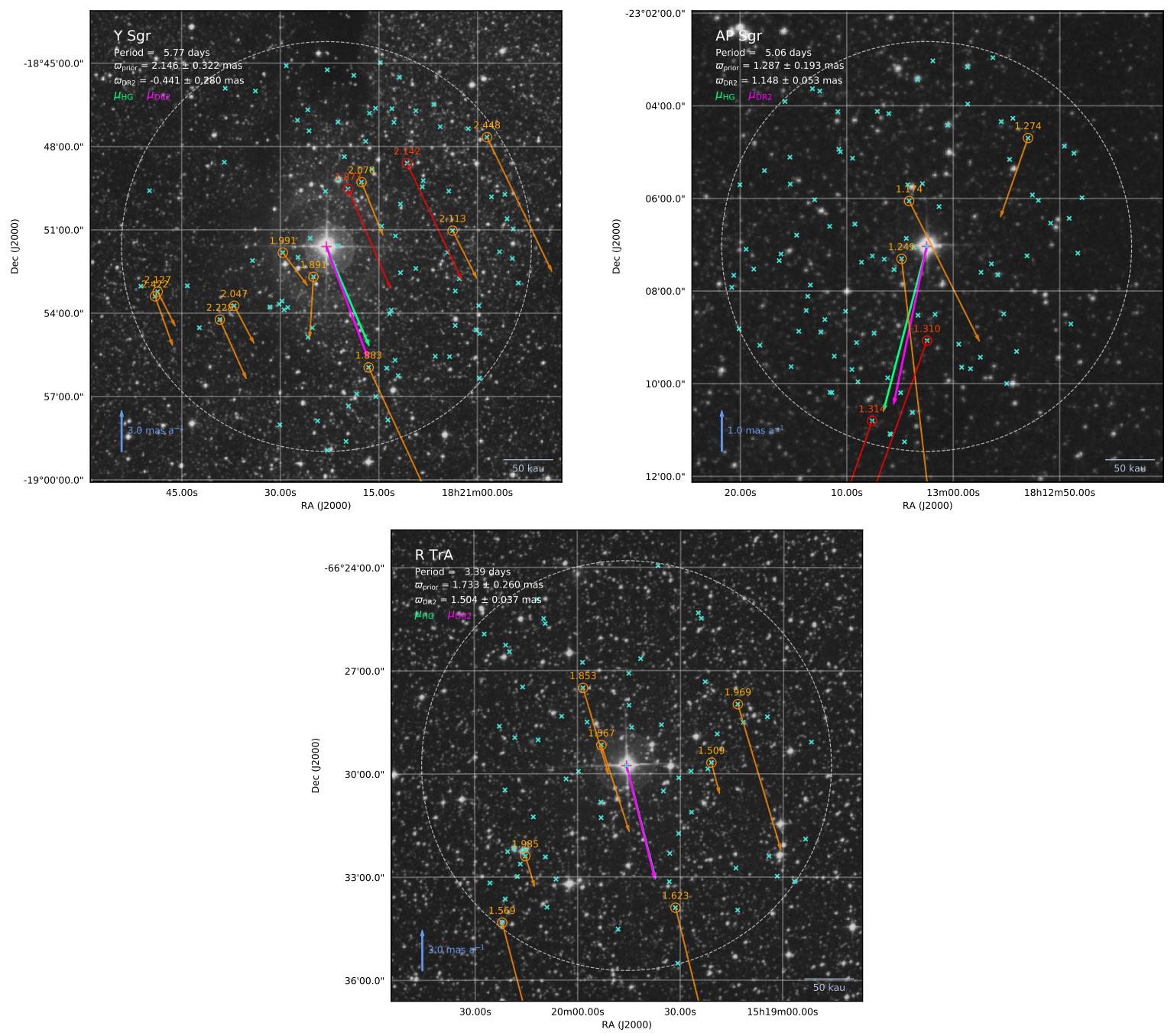

Fig. A.8. Same as Fig. A.7 for Y Sgr, AP Sgr and R TrA. 
P. Kervella et al.: Multiplicity of Galactic Cepheids and RR Lyrae stars from Gaia DR2. II.

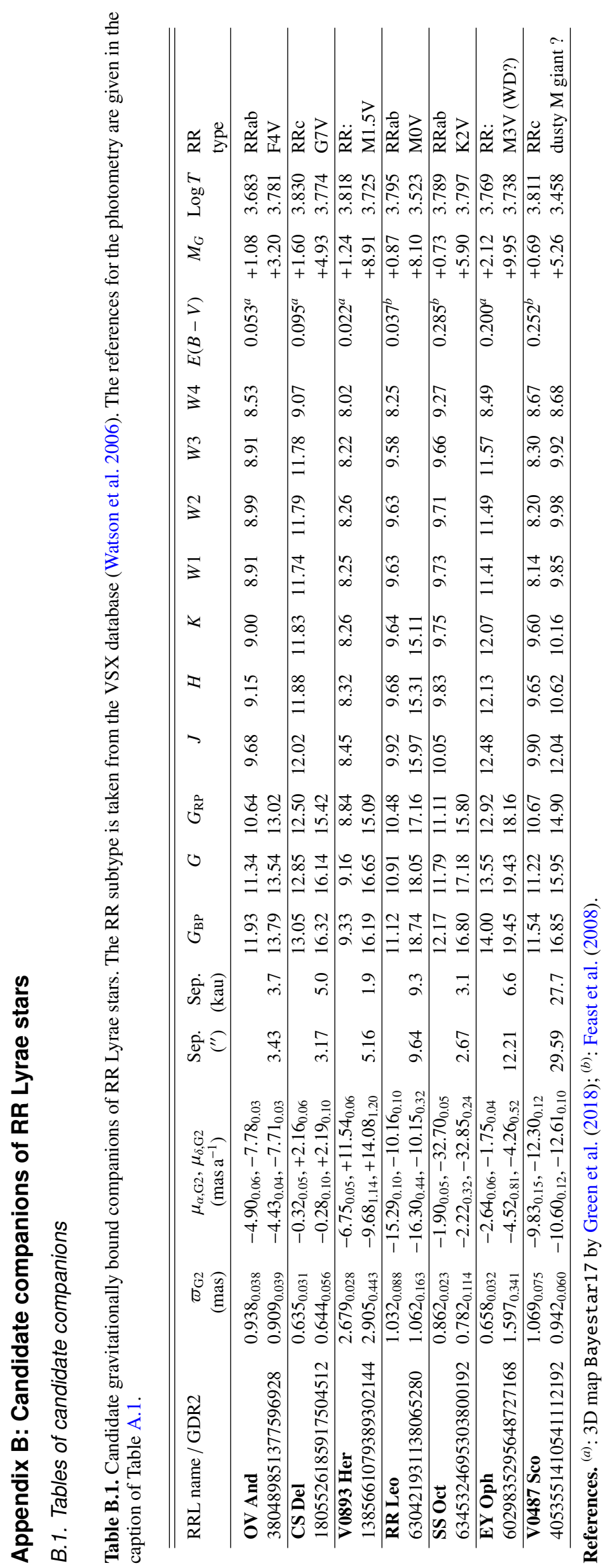




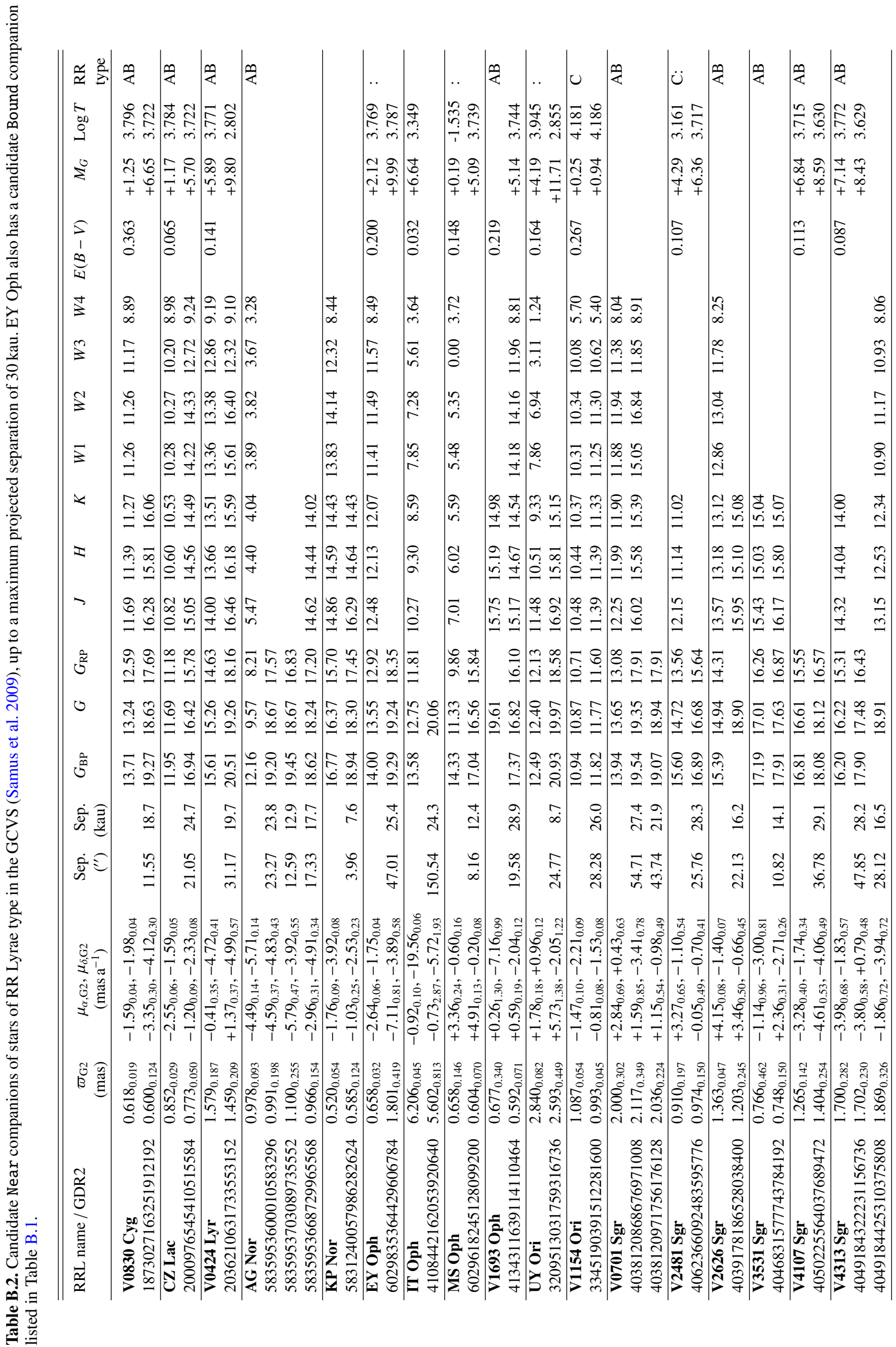


P. Kervella et al.: Multiplicity of Galactic Cepheids and RR Lyrae stars from Gaia DR2. II.

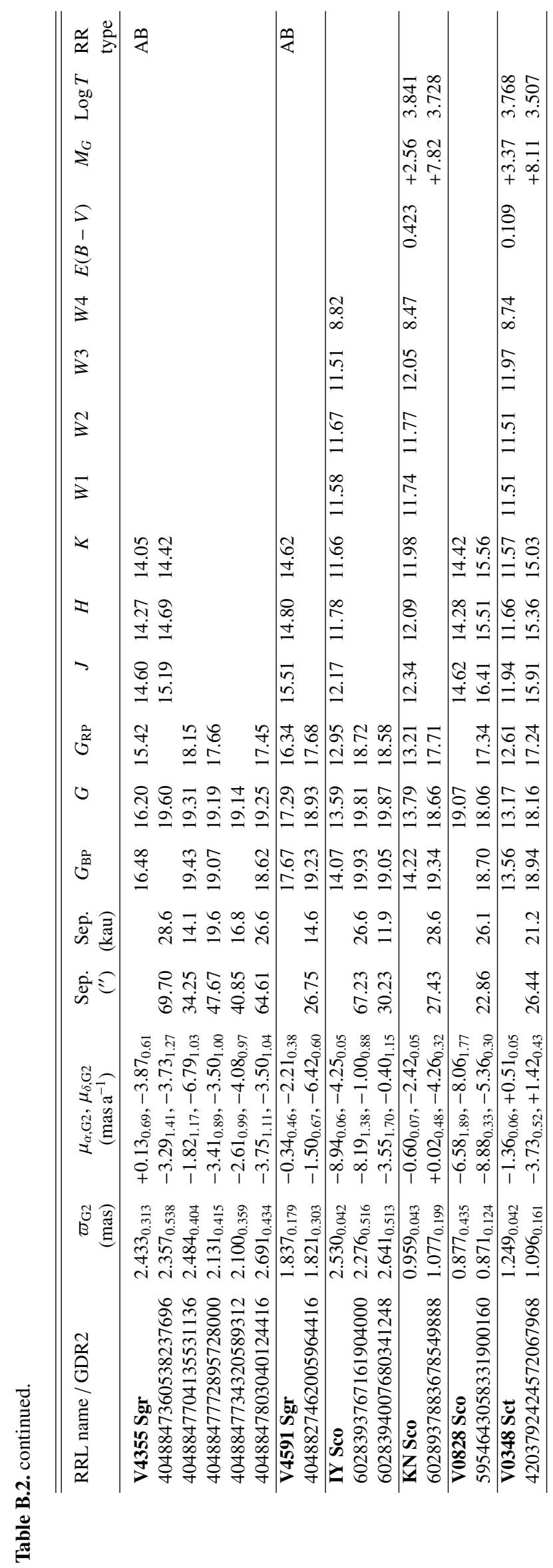




\section{B.2. Field charts}

The fields surrounding the RRLs with detected Bound candidate companions, and a selection of RRLs with Near candidate com- panions are presented in Figs. B.1-B.3. The adopted symbols are described in Sect. A.2.
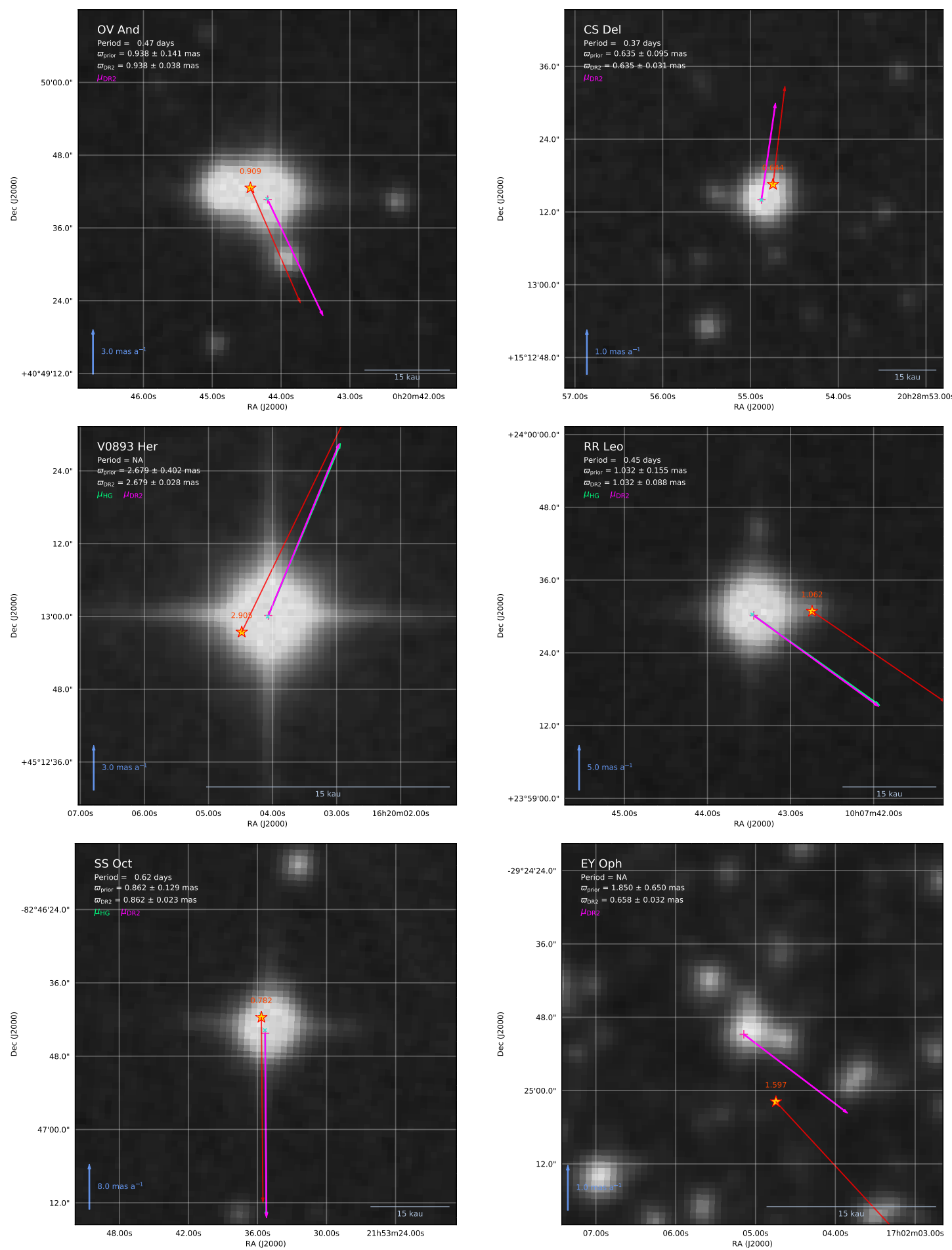

Fig. B.1. Field charts for RR Lyrae stars with Bound candidate companions: OV And, CS Del, V0893 Her, RR Leo, SS Oct and EY Oph. 
P. Kervella et al.: Multiplicity of Galactic Cepheids and RR Lyrae stars from Gaia DR2. II.

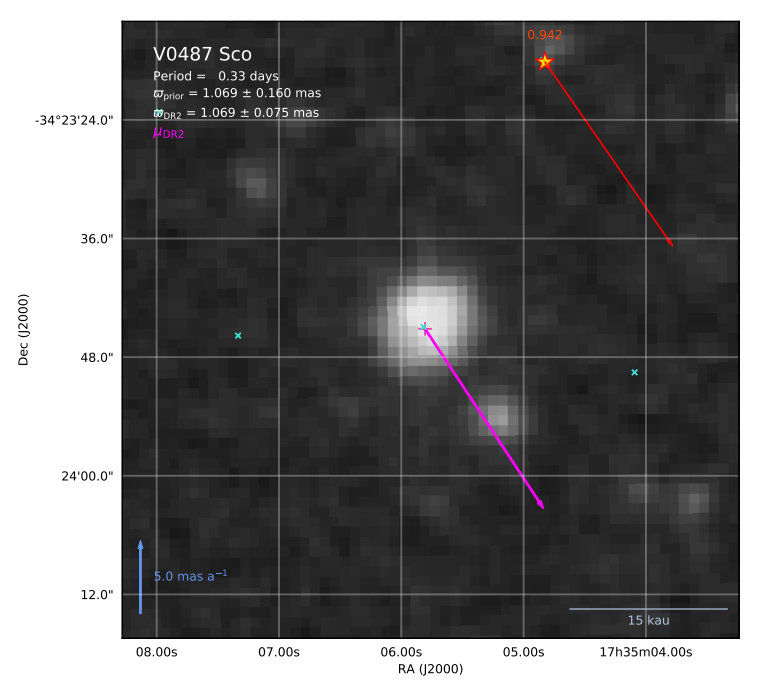

Fig. B.2. Same as Fig. B.1 for V0487 Sco. 

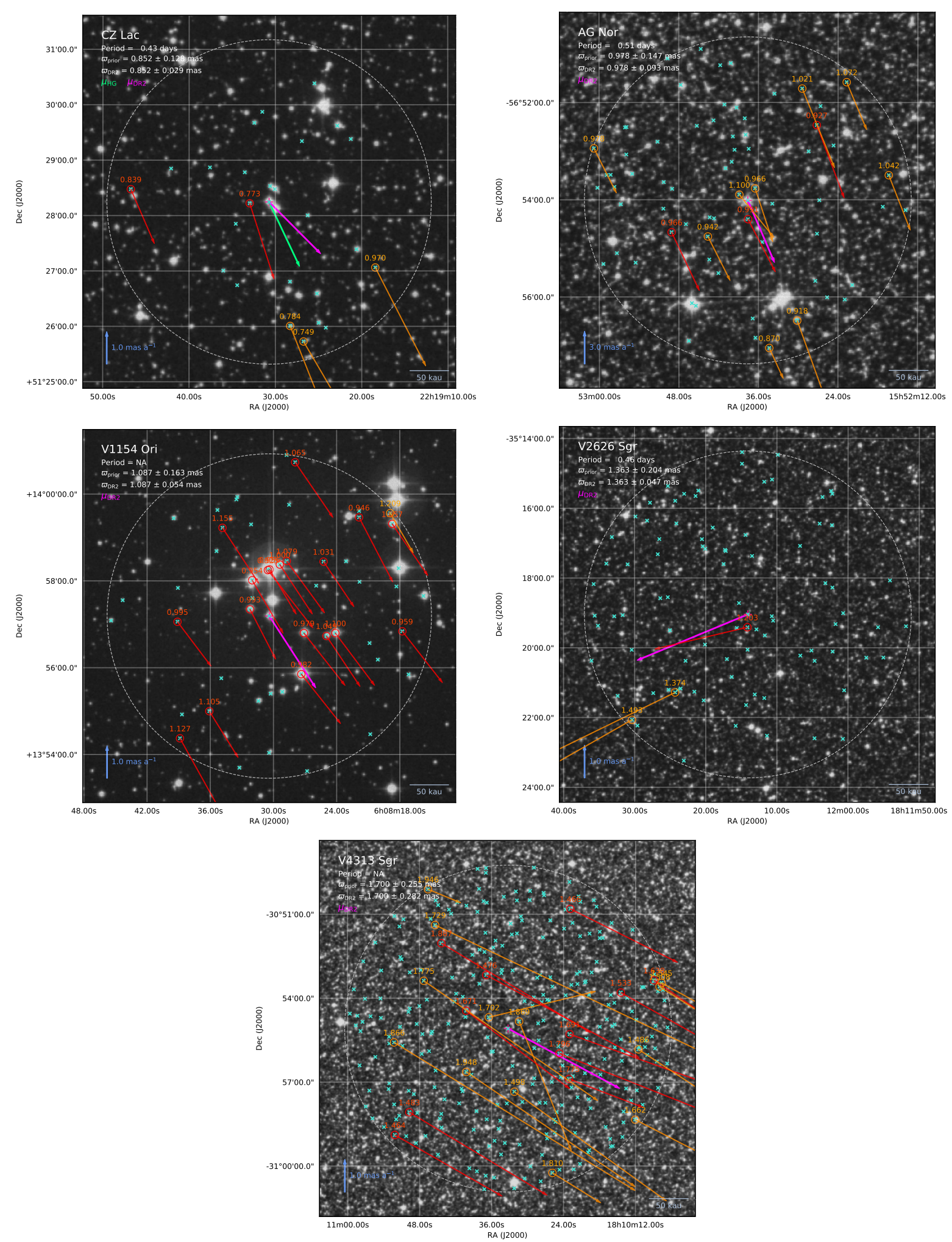

Fig. B.3. Field charts for selected RR Lyrae stars with Near candidate companions: CZ Lac, AG Nor, V1154 Ori, V2626 Sgr and V4313 Sgr. 
P. Kervella et al.: Multiplicity of Galactic Cepheids and RR Lyrae stars from Gaia DR2. II.

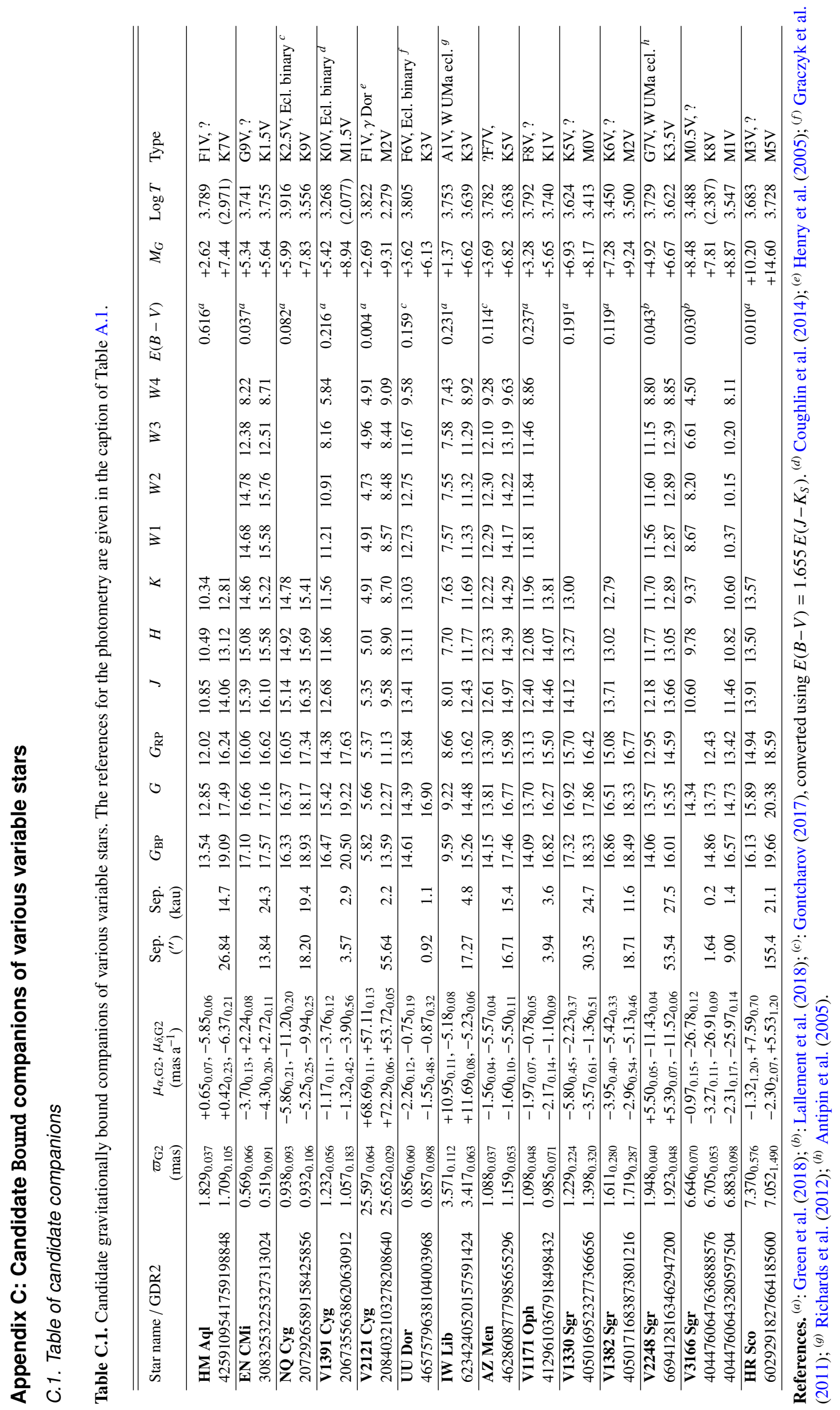




\section{C.2. Field charts}

The fields surrounding the variable stars of various classes with detected Bound candidate companions are presented in Figs. C.1-C.3. The adopted symbols are described in Sect. A.2.
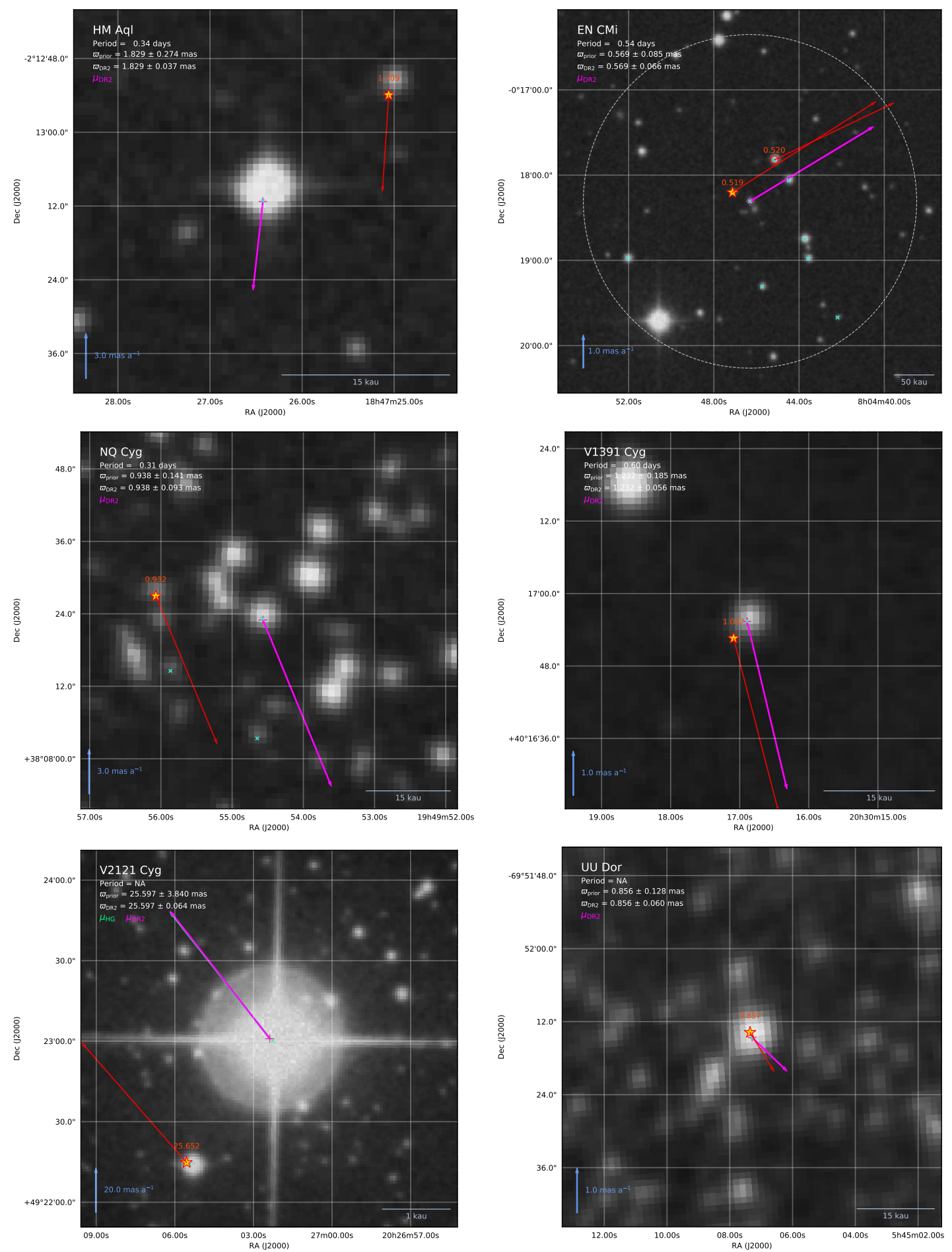

Fig. C.1. Field charts for various variable stars with Bound candidates: HM Aql, EN CMi, NQ Cyg, V1391 Cyg, V2121 Cyg and UU Dor. 
P. Kervella et al.: Multiplicity of Galactic Cepheids and RR Lyrae stars from Gaia DR2. II.
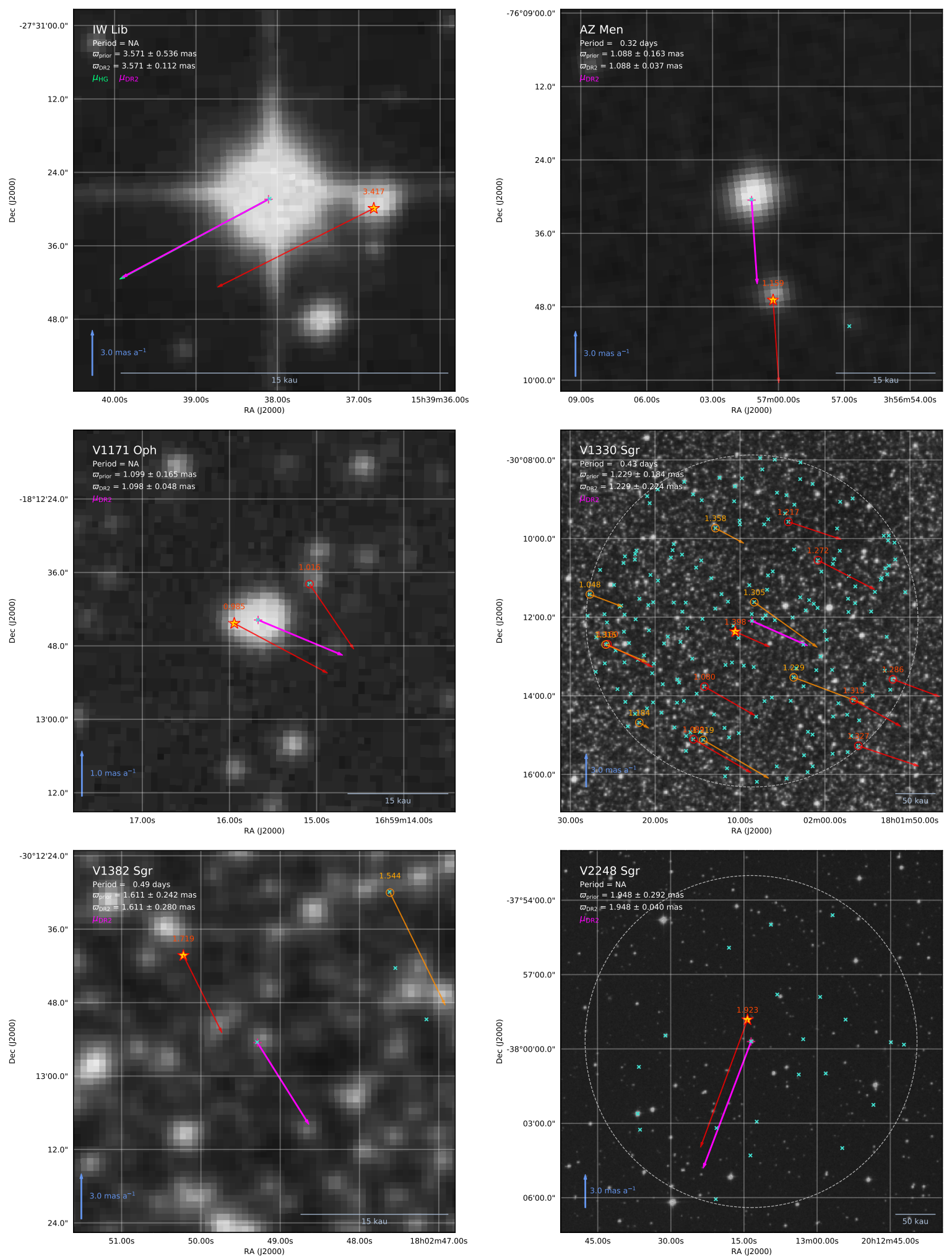

Fig. C.2. Same as Fig. C.1 for IW Lib, AZ Men, V1171 Oph, V1330 Sgr, V1382 Sgr and V2248 Sgr. 
A\&A 623, A117 (2019)
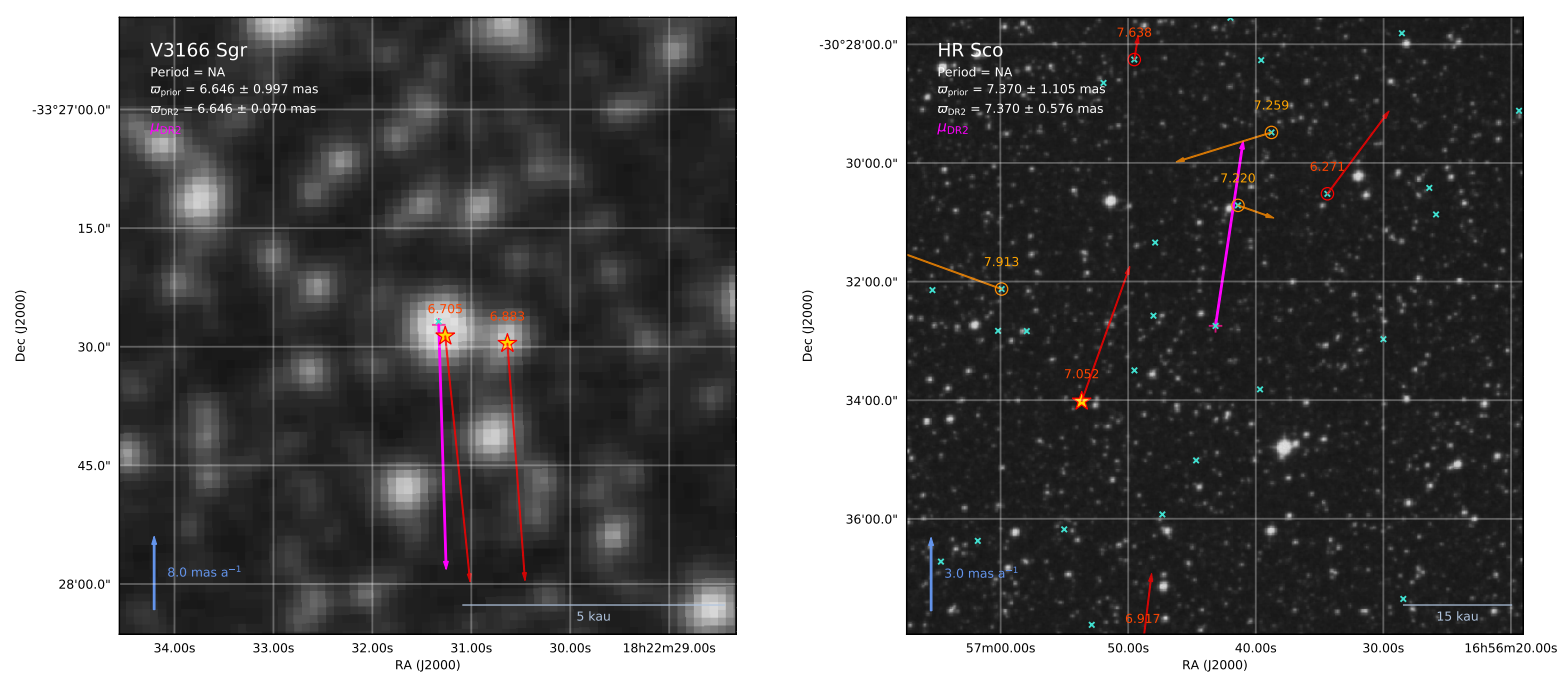

Fig. C.3. Same as Fig. C.2 for V3166 Sgr and HR Sco. 